\title{
Coherent structures in turbulent Rayleigh-Bénard convection
}

Dissertation

zur Erlangung des Doktorgrades der Mathematisch-Naturwissenschaftlichen Fakultäten der Georg-August-Universität zu Göttingen

Vorgelegt von

Tomi Haramina

aus Zagreb, Kroatien

Göttingen 2005 
D7

Referent: Prof. Dr. Andreas Tilgner

Korreferent: PD Dr. Martin Rein

Tag der mündlichen Prüfung: 5. Januar 2006 


\section{Contents}

1 Introduction $\quad 8$

2 Theoretical background $\quad 11$

2.1 Governing equations . . . . . . . . . . . . . . 11

2.2 Stability problem . . . . . . . . . . . . . 12

2.3 Turbulence . . . . . . . . . . . . . . . . . 15

2.4 Coherent structures . . . . . . . . . . . . . 16

3 Experimental setup and methods $\quad 18$

3.1 Cells . . . . . . . . . . . . . . . . . 18

3.2 Thermistors and temperature measurement . . . . . . . . 19

4 Coherent structures in the boundary layers $\quad \mathbf{2 2}$

4.1 Experiment . . . . . . . . . . . . . . . 22

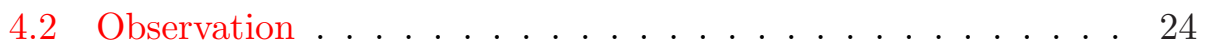

4.3 Mechanism of streak formation . . . . . . . . . . 25

4.3.1 Linear stability analysis . . . . . . . . . . 26

4.3.2 Stochastic process . . . . . . . . . . . 30

4.3.3 Comparison with streaks in isothermal shear flows . . 34

4.4 Outlook . . . . . . . . . . . . . . . . . 35

5 Coherent structures in the bulk $\quad 37$

5.1 Experimental setup . . . . . . . . . . . . . 37

5.2 Some important scales in turbulent convection . . . . . . 39

5.3 Large scale circulation . . . . . . . . . . . . . . . . . 41

5.3 .1 Coherency . . . . . . . . . . . . . . . 44 43

5.3 .2 Spatial modes . . . . . . . . . . . . . . 45

5.4 Coherent structures on intermediate scales . . . . . . . . 45

5.4 .1 Two prominent scales . . . . . . . . . . 50

5.4 .2 Temperature as passive scalar . . . . . . . . . . 54

5.4 .3 Taylor hypothesis . . . . . . . . . . . . . . 57

5.5 Overview of scales in the flow . . . . . . . . 60 
6 Influence of the container shape on the flow 61

6.1 Experiments........................ 6 61

6.2 Velocity field . . . . . . . . . . . . . . . 63

6.3 Temperature field . . . . . . . . . . . . . . . 64

6.4 Conclusion and outlook . . . . . . . . . . . . 68

$\begin{array}{lll}7 & \text { Summary } & \mathbf{7 0}\end{array}$

$\begin{array}{ll}\text { A Technical data } & \mathbf{7 2}\end{array}$

B Computer programs $\quad \mathbf{7 5}$

B.1 Hardware Control . . . . . . . . . . . . . . 75

B.2 Data Analysis . . . . . . . . . . . . . . . 75

$\begin{array}{ll}\text { C Flow visualization with thermochromic liquid crystals } & \mathbf{8 0}\end{array}$ 


\section{List of Tables}

4.1 Minimal streak separation $\delta$ determined for different Ra and Pr according to the procedure described in the text. . . . . 34

5.1 Parameters used in the fit shown in Fig. (5.9). Distance from the plate is normalized with the height of the cell. . . . . . 50

5.2 Overview of the scales discussed in the thesis. . . . . . . . 60

6.1 Comparison of the ratios of the rms' in given experimental setups calculated from (6.3) and the ones measured directly. . 68 


\section{List of Figures}

1.1 Gravity anomalies in the Southeast Pacific derived from ERS1 Geodetic Mission data. Note the elongated anomalies perpendicular to the East Pacific Rise crossing troughs and older fracture zones to the west continuously. Note the increase in wavelength away from the mid-ocean ridge. (Picture and caption from Marquart et al. (1999). Reprinted with kind permission from Blackwell Publishing Ltd.) . . . . . . . . . . . 9

2.1 Régime diagram in Ra-Pr parameter space according to Krishnamurti (1970b) and Busse (1981). The dashed line is the result of somewhat inconsistent measurements (Krishnamurti, 1970b). Points denote parameters used in the thesis. . 14

2.2 Schematic sketch of the temperature profile in the RayleighBénard convection: linear profile of the conduction state (dashed line) and a profile in a turbulent state (solid line). . . . . . 16

2.3 Structures in a convection cell according to Zocchi et al. (1990). 17

3.1 Photography of the cubical cell used in the experiments. . . . 19

3.2 Schematic picture of the temperature measurement process in a box filled with water (1). A thermistor (2) is connected to the Wheatston bridge (3) as a resistance arm. As the temperature generally oscillates the bridge is unbalanced, that is the voltage (4) on the bridge oscillates. The voltage is measured and transported to the computer using PCI Bus Data Acquisition Board (5). The time-voltage pairs are saved to the computer disc (6). From the voltage data the resistivity of the thermistor is calculated in each moment and finally from the resistivity the temperature is determined using the calibration data. . . . . . . . . . . . . . . 20

4.1 View of the bottom plate. The line AB is the main diagonal of the plate perpendicular to the mean large scale flow (which is from the lower left to the upper right corner. A few streaks are marked with arrows. . . . . . . . . . . 23 
4.2 Photograph of the lower plates processed with "StreakView" software. The mean large scale flow is from the upper left to the lower right corner. . . . . . . . . . . . . . . . . . 24

4.3 Visualization of the flow over the bottom plate, with dye layer thicker then the boundary layers. The large scale mean flow is from the lower left to the upper right corner. The "waves" sketched in Fig. (2.3) are here clearly visible in the upstream part of the flow (circular structures) . . . . . . . . 25

4.4 The mean temperature as function of distance $\mathrm{z}$ from the top plate for $\mathrm{Ra}=1.3 \cdot 10^{9}$ and $\operatorname{Pr}=6.7$. The intersection of the solid lines defines the thickness of the boundary layer (see text). 26

4.5 Two experimental histograms of streak separations. Lefthand figure shows the separations across the diagonal CD and is based on 134 photographs showing a total of 1277 streaks. The righthand figure shows the histogram for the main diagonal $\mathrm{AB}$ and is based on 56 pictures showing the total of 305 streaks. . . . . . . . . . . . . . 27

4.6 Model used for the calculation of the most unstable mode using linear stability analysis. The velocity is scaled with its maximal value, temperature with the temperature difference between the value at the top of the computational domain and the value at the plate and the length is scaled with the half of the height of the computational domain. . . . . . . . 28

4.7 Measured temperature profile for $\mathrm{Ra}=1.3 \cdot 10^{9}, \operatorname{Pr}=6.6$ (diamonds) and aproximation used for some computations (solid line). Temperature and length are nondimensionaled (see Fig.(4.6)). $T^{*}$ is the temperature at the plate. . . . . . .

4.8 The value of the wavelength of the most unstable mode (diamonds) becomes independent of the value of $z_{\max }$ and saturates to $8.4 \mathrm{~mm}$ (solid line). . . . . . . . . . . 30

4.9 Geometry of the problem described in the text. . . . . . . . . 31

4.10 The bar chart is a histogram of the streak separation on the line CD in Fig. (4.1), and the solid line is the theoretical curve given by Eq. (4.9) (for $\Delta D=0.2 \mathrm{~mm}, \delta=6 \mathrm{~mm}$ and $p=0.82) \ldots \ldots \ldots \ldots \ldots \ldots \ldots$

4.11 The bar chart is the probability distribution for the number of streaks $n$ visible in any one photograph on the line $\mathrm{CD}$ for the same data as in Fig. (4.10). The diamonds are $P(n, L)$ according to Eq. (4.10) and (4.11) for $L=198 \mathrm{~mm}, \delta=6$ $\mathrm{mm}$ and $p=0.82 \ldots \ldots \ldots \ldots \ldots$

4.12 Temperature signatures of streaks measured at the distance of about $1 / 8$ of the thermal boundary layer thickness from the plate. . . . . . . . . . . . . . 
5.1 Schematic view of the upper plate with a thermistor hold by the translation table. . . . . . . . . . . . . 38

5.2 Positions of thermistors used for simultaneous temperature measurements and their labels used in text. Thermistors are at distances $20 \mathrm{~mm}$ one from another, thermistors 1 and 9 are at $20 \mathrm{~mm}$ from the upper and lower plates, respectively.

5.3 Temperature dissipation spectrum at position $20 \mathrm{~mm}$ from the plate. The narrow peak at high frequency end is aliased $50 \mathrm{~Hz}$ noise from the electrical network. . . . . . . . . . 40

5.4 Histograms of the temperature fluctuations (averaged over symmetrical positions) from the local mean. Positions are labeled as described in Fig. (5.2). . . . . . . . . . . . . . 42

5.5 Arbitrary chosen sections of the temperature time series 20 $\mathrm{cm}$ from the upper plate (left) and $20 \mathrm{~cm}$ from the lower plate. 43

5.6 Power spectrum of the temperature fluctuation at $40 \mathrm{~mm}$ under the center of the upper plate. . . . . . . . . . . 44

5.7 Coherence and phase of the thermistors placed $20 \mathrm{~mm}$ beneath the upper plate and the one placed $20 \mathrm{~mm}$ above the lower plate. . . . . . . . . . . . . . . . 4 44

5.8 Eigenvectors (left) and the power spectra of the corresponding modes. . . . . . . . . . . . . . . 46 46

5.8 (continued) Eigenvectors (left) and the power spectra of the corresponding modes. . . . . . . . . . . . . 47

5.8 (continued) Eigenvectors (left) and the power spectra of the corresponding modes. . . . . . . . . . . . . 48

5.9 Spatial correlations of the temperature at (from top to bottom) $20 \mathrm{~mm}, 60 \mathrm{~mm}$ and $100 \mathrm{~mm}$ from the plate (points) and the fit to the sum of two exponential functions given by (5.11) (solid line) in semi-log scale. . . . . . . . . . . . . . 51

5.10 Visualization of the flow in the upper half of the cell using thermocrystals. With "A", "B" and "C" are denoted the eddies with size $L_{2}$. For details see the text. The vertical bright line is the holder for thermistors positioned along the central axis of the cell. . . . . . . . . . . . . 52

5.11 Dependence of the size of the eddies on the distance from the plate. . . . . . . . . . . . . .

5.12 Relative contribution of the smaller eddies to the total power as dependent on the distance from the plate. . . . . . . . . 54

5.13 Cliff-ramp structures in the temperature time series measured in the cell center. . . . . . . . . . . . 56

5.14 Typical size of a cliff-ramp structure in a part of the temperature time series. Horizontal dashed lines denote rms temperature at the point. . . . . . . . . . 56 
5.15 Measured power spectra (diamonds) and the power spectra given by (5.23) (solid lines) at positions, top to bottom, 20 $\mathrm{mm}, 60 \mathrm{~mm}$ and $100 \mathrm{~mm}$ from the plate. . . . . . . 58

5.16 Velocity $v$ used to fit the power spectra (5.23) to the measured power spectra scaled down with the factor of 4 (diamonds) and the root mean square of the vertical velocity fluctuations in a similar cell at the same Rayleigh number from Tilgner et al. (1993) (crosses). Velocities are made nondimensional using the height of the cell and the thermal diffusivity, and the distance from the plate is scaled with the height of the cell. 59

6.1 Influence of the container tilting on the direction of the flow in a cube. In the leveled cube the flow is across the diagonal on the plates (left) and in the tilted cube the flow is parallel to the sides. . . . . . . . . . . . . . . 62

6.2 Example of the horizontal oscillation of the large scale mean flow in the cylinder. In the course of 8 minutes the flow changed direction for almost $90^{\circ} \ldots \ldots \ldots$. . . . . . 63

6.3 Root mean square profiles in the cube (squares) and the cylinder (circles). . . . . . . . . . . . . . . . . . 64

6.4 Root mean squares in the leveled cubical and cylindrical containers and the containers inclined for $2^{\circ}$ and $4^{\circ}$ at positions 1 to $5 \ldots \ldots \ldots \ldots \ldots \ldots \ldots$

6.5 Histograms of the temperature oscillations at position 6 in different experimental setups. . . . . . . . . . . . 66

6.6 Power spectra of the temperature time series in different cells. Each spectrum is average of the spectra at symetrical positions 1 and $9 \ldots \ldots \ldots \ldots \ldots \ldots \ldots$

6.7 Dependence of $\operatorname{rms}(T)$ on $\sqrt{\epsilon_{\text {bulk }}}$. Straight line is a linear fit. 68

B.1 RBScan, setup window. . . . . . . . . . . 76

B.2 RBScan, conversion of voltage measured on the Wheatstone bridges to temperature. . . . . . . . . . . . . 77

B.3 RBScan as interface to other programs. . . . . . . . 77

B.4 RBScan, setup for Fourier transformation. . . . . . . . . 78

B.5 StreakView graphycal user interface. . . . . . . . . . 79

C.1 Flow visualization in the part of the upper half of the cell. The bright vertical line is the thermistor holder. . . . . . . . 81 


\section{Chapter 1}

\section{Introduction}

$T^{\text {HE }}$ phenomenon of the air that rises over hot surfaces must have been

1 observed already in the early stages of human's interest in the natural phenomena. However, it was not until 1900 that someone scientifically coped with the problem - Henri Bénard was the first who, in the frame of his doctoral thesis, undertook systematic experimental research of the layer of fluid heated from below (Bénard, 1901). In this experiment he observed the regular cellular pattern of hexagonal convection cells. In 1916 John William Strutt, Lord Rayleigh, explained the phenomenon in terms of a buoyancy driven instability (Lord Rayleigh, 1916). Although we know today that the pattern observed in Bénard's experiment was caused by temperature dependent surface tension rather than by buoyancy, the buoyancy driven convection in the layer of fluid heated from bellow and cooled from above is today referred to as "Rayleigh-Bénard convection". Surface tension induced convection is nowadays known as "(Bénard-)Marangoni convection".

Motivation for investigation of Rayleigh-Bénard convection is manifold. In nature thermal convection is an omnipresent phenomenon on a wide range of scales - in the Earth's core and mantle, atmosphere, oceans and stars. In industrial applications whenever heat transfer must be considered thermal convection enters in various forms - in nuclear reactors, in crystallisation processes, in solar heating devices. Most of the flows mentioned are (or can be) turbulent. On a more fundamental level laboratory experiments on thermal convection have attracted attention as particularly simple systems, where transition to turbulence and its properties can be studied (Busse, 1978).

One striking property of a turbulent flow is appearance of patches of ordered structures on the otherwise turbulent background. The phenomenon is interesting per se, as it implies some degree of order in otherwise disordered turbulent flow. But the motivation for the research of the structures can also be found in both technical applications as well as in nature. An example of technical application is the research of the influence of the flow structures 


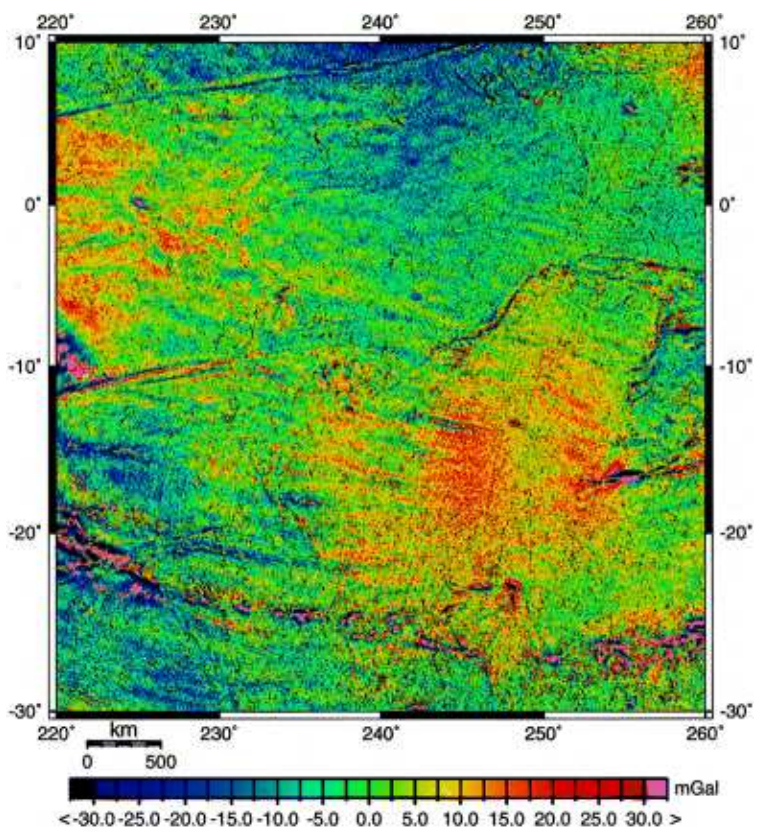

Figure 1.1: Gravity anomalies in the Southeast Pacific derived from ERS-1 Geodetic Mission data. Note the elongated anomalies perpendicular to the East Pacific Rise crossing troughs and older fracture zones to the west continuously. Note the increase in wavelength away from the mid-ocean ridge. (Picture and caption from Marquart et al. (1999). Reprinted with kind permission from Blackwell Publishing Ltd.)

(on all scales) on the comfort of passengers in cabins of airplanes and trains.

The small scale structures in geophysical context were found in the gravity anomaly field below the cooling oceanic lithosphere as shown in Fig. (1.1) (Marquart et al., 1999). These structures could be explained by the results presented in Chapter 4.

This work will give answers to some questions about the properties of turbulent Rayleigh-Bénard convection regarding spatio-temporal organization of turbulent structures on different scales, their influence on the general dynamics of the flow and geometry dependence of the structures and the flow itself. Organized structures in the velocity and temperature field distinguished from the turbulent background are already found in different types of turbulent flows. In thermal convection thermal plumes present a well known example. In this thesis a new type of organized structure is discovered inside the boundary layers of convective turbulent flow. Its characteristics are presented and the mechanism of its formation proposed. Further, using different time series analysis methods new information is gathered about the organization of the flow on large and intermediate scales in the bulk. These pieces of information are collected by means of laboratory experiments in tanks with two geometries - cubical and cylindrical. Finally, 
the influence of the shape of the container on the flow is investigated and it is shown that there are significant effects of the shape on the flow which are often overlooked in literature.

The thesis is organized as follows: In Chapter 2 the theoretical background of the problem is given. In Chapter 3 the basic experimental setup and methods are described. Their specific variations will be detailed in the parts where they were used (this refers mostly to the arrangement of temperature probes in the cells). Chapter 4 deals with the small scale coherency in the boundary layers - visualization method is presented which served to discover a new type of the structures in the thermal turbulent convection, their statistical characteristics are presented and possible mechanisms of their production are discussed. Also, the structures are compared with the small scale structures in isothermal flows. In Chapter 5 coherent structures in the bulk are studied. It is shown that, contrary to the traditional cascade picture of the turbulence, only two scales dominate the flow. In Chapter 6 the effect of the shape of the container is investigated and it is found that the very center of the cell can feel the influence of the wall shape, even if all other experimental conditions are left the same. This is an important result because in the literature the experimental results are often compared without regard to the shape of the container. In Chapter 7 the results of the thesis are summarized. The thesis ends with three appendices. Appendix A contains technical data about the equipment used in the experiments. In Appendix B some more important computer programs programmed in the course of the thesis are listed. Finally, Appendix C contains photographs from visualization of the flow using thermochromic liquid crystals. 


\section{Chapter 2}

\section{Theoretical background}

$\mathrm{T}^{\mathrm{N}}$ this chapter only the general theoretical background is given as a frame1 work for the thesis. The theoretical and experimental results are presented later in the respective chapters.

\section{$2.1 \quad$ Governing equations}

The exact equations for a heat conducting viscous fluid in the gravity field can be simplified in a wide range of applications without significant influence on the final results. Oberbeck (1879) and Boussinesq (1903) independently derived approximations of the equations for the flows in which because of small temperature variations all fluid properties $\left(c_{V}\right.$ - specific heat at constant volume, $\nu$ - kinematic viscosity, $\alpha$ - coefficient of volume expansion and $k$ - coefficient of thermal conduction) are assumed constant, except for density when it causes buoyancy. Using the results of the analysis of the validity of the Boussinesq-Oberbeck approximation carried out by Gray and Giorgini (1976) it can be shown that for the parameter range used in this work the approximation is valid. On the basis of this approximation for small $\left(\leq 10^{\circ} \mathrm{C}\right)$ temperature differences between the bottom and the top of the fluid layer the density $\rho$ is

$$
\rho=\rho_{0}\left\{1-\alpha\left(\theta-\theta_{0}\right)\right\},
$$

where $\rho_{0}$ is the density of the fluid at the temperature $\theta_{0}$ of the bottom of the layer and $\alpha$ is the constant coefficient of volume expansion.

The equations of motion follow from the law of the conservation of momentum and are:

$$
\partial_{t} \vec{u}+(\vec{u} \cdot \nabla) \vec{u}=-\nabla P+\nu \nabla^{2} \vec{u}-g \alpha\left(\theta_{0}-\theta\right) \hat{k},
$$

where $g$ is the magnitude of the acceleration due to gravity, $P=p / \rho_{0}+\Phi$, $p$ being the isotropic pressure at the given position when there is no strain, 
$\Phi$ the gravitational potential $(\vec{g}=-\nabla \Phi), \vec{u}$ is the velocity vector, $\nu$ the kinematic viscosity and $\hat{k}$ the unit vector with direction $-\vec{g}$.

The continuity equation expresses the conservation of mass. In the light of Boussinesq-Oberbeck approximation the fluid is considered incompressible so the continuity equation reads:

$$
\nabla \cdot \vec{u}=0 .
$$

Finally, the energy (heat) equation expresses the law of the conservation of energy. Assuming that the internal heating is negligible it reads:

$$
\partial_{t} \theta+(\vec{u} \cdot \nabla) \theta=\kappa \nabla^{2} \theta,
$$

where $\kappa=k / \rho_{0} c_{v}$ is the thermal diffusivity.

The equations (2.2) - (2.4) are the basic hydrodynamic equations. Supplemented by the equation of state (2.1) they form the basic system used in the fluid dynamics if Boussinesq-Oberbeck approximation is valid.

\subsection{Stability problem}

Consider an infinite horizontal layer of fluid in which a steady adverse temperature gradient is maintained and there are no motions

$$
\theta \equiv \theta(z) \quad \text { and } \quad \vec{u}=0 .
$$

The pressure distribution is then governed by the equation

$$
\partial_{z} p=\rho \vec{g} \hat{k},
$$

where $\rho$ is given by (2.1). From (2.4) it follows that the temperature distribution is governed by the equation

$$
\nabla^{2} \theta=0 .
$$

The solution of $(2.7)$ is

$$
\theta=\theta_{0}-\beta z,
$$

where $\beta$ is the adverse temperature gradient which is maintained. For the density it follows from (2.8) and (2.1)

$$
\rho=\rho_{0}(1+\alpha \beta z) .
$$

Equation (2.6) can now be integrated to give

$$
p=p_{0}-g \rho_{0}\left(z+\frac{1}{2} \alpha \beta z^{2}\right) .
$$


Let now the initial state given by equations (2.5), (2.8), (2.9) and (2.10) be slightly perturbed, and let $\vec{u}^{\prime} \equiv\left(u^{\prime}, v^{\prime}, w^{\prime}\right), \theta^{\prime}$ and $p^{\prime}$ denote small perturbations of velocity, linear temperature profile and pressure, respectively. By ignoring terms of second and higher orders in the perturbations, equations (2.2), (2.4) and (2.3) give

$$
\begin{gathered}
\partial_{t} \vec{u}^{\prime}=-\nabla\left(\frac{p^{\prime}}{\rho_{0}}\right)+\nu \nabla^{2} \vec{u}^{\prime}+g \alpha \theta^{\prime} \hat{k}, \\
\partial_{t} \theta^{\prime}=\beta w^{\prime}+\kappa \nabla^{2} \theta^{\prime}, \\
\nabla \cdot \vec{u}^{\prime}=0 .
\end{gathered}
$$

It proves convenient to make these equations nondimensional. One possible way to do it (the choice of scales is not unique) is to choose the distance between the plates $L$ as the length scale, the thermal diffusion time $L^{2} / \kappa$ as the time scale and the difference of the plates' temperatures $\Delta \theta \equiv \beta L$ as the temperature scale. Then the equations describing the problem are

$$
\begin{gathered}
\partial_{t} \vec{u}=-\nabla p+\operatorname{Pr} \nabla^{2} \vec{u}+\operatorname{Ra} \operatorname{Pr} \theta \hat{k}, \\
\partial_{t} \theta-w=\nabla^{2} \theta, \\
\nabla \cdot \vec{u}=0
\end{gathered}
$$

where all variables are nondimensional and

$$
\mathrm{Ra}=\frac{g \alpha \Delta \theta L^{3}}{\kappa \nu}
$$

is the nondimensional Rayleigh number and

$$
\operatorname{Pr}=\frac{\nu}{\kappa}
$$

is the nondimensional Prandtl number. The appropriate boundary conditions for rigid boundaries are

$$
w=\frac{\partial w}{\partial z}=\theta=0 \quad \text { at } \quad z=0 \quad \text { and } \quad z=1 .
$$

It is now possible to perform the stability analysis in terms of normal modes, and finally find the critical values of the nondimensional parameters for the flow to become unstable and the wavelength of the most unstable mode (e.g. Chandrasekhar, 1981). The result of the procedure is that independently of the Prandtl number the critical Rayleigh number is $\mathrm{Ra}_{c} \approx 1708$.

The stability analysis becomes very complicated at the parameter space away from the onset of the instability as the nonlinear effects become important. Nevertheless, the large number of experiments on the subject deliver a quite satisfactory picture of the flow types for different regions in the parameter space. This knowledge is summarized in Fig. (2.2). Additionally (not shown in the picture), Castaing et al. (1989) found for the Helium gas at 


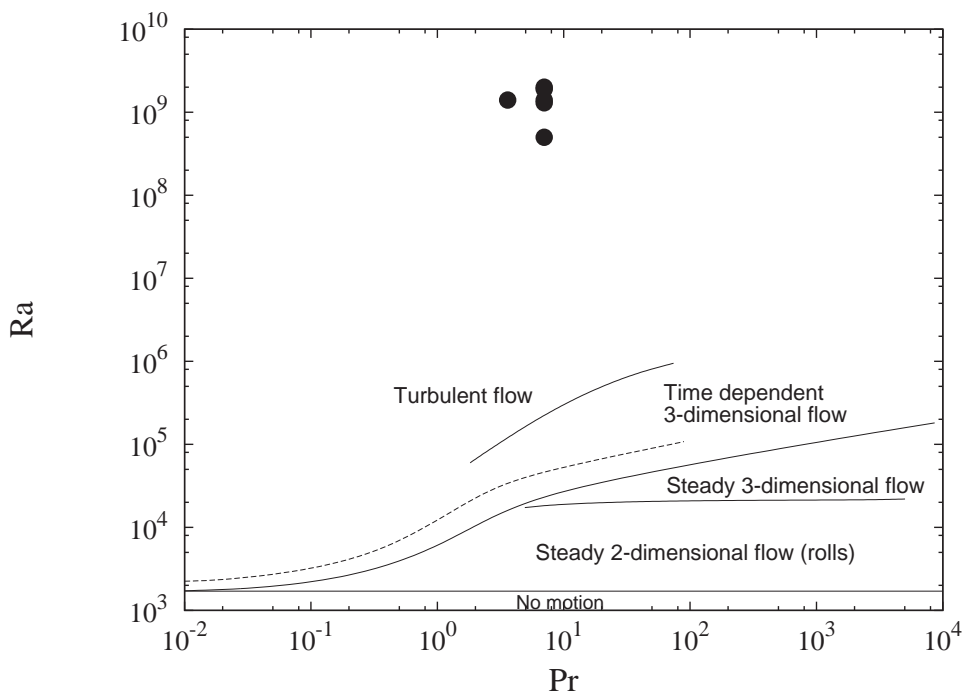

Figure 2.1: Régime diagram in Ra-Pr parameter space according to Krishnamurti (1970b) and Busse (1981). The dashed line is the result of somewhat inconsistent measurements (Krishnamurti, 1970b). Points denote parameters used in the thesis.

roughly $5 \mathrm{~K}$ (Prandtl number between 0.65 and 1.5) two turbulent régimes which they named "soft" and "hard" turbulence. The distinction is based on different scaling of the heat transport with Rayleigh number, which coincides with different distribution of local temperature fluctuations in the center of the container. The soft turbulence occurs for Rayleigh numbers $10^{6}<\mathrm{Ra}<4 \cdot 10^{7}$ and has a Gaussian distribution and the hard turbulence occurs for $4 \cdot 10^{7}<\mathrm{Ra}<10^{13}$ and has exponential tails in the distribution.

An interesting feature of the thermal convection is that by increasing $\mathrm{Ra}$ for given Pr the flow undergoes a number of discrete transitions, remaining in each régime for a finite range of Rayleigh numbers (Krishnamurti, 1970a). The transition to turbulence through a number of régimes is a somewhat special feature of the Rayleigh-Bénard convection (shared with Couette flow and flow between spheres when the outer cylinder or sphere is at rest, respectively) as in many other flows (like Poisseuille flow through the annulus between the two long concentric cylinders, pipe flow, Couette flow with inner cylinder at rest) the transition from laminar to turbulent flow is direct (Busse, 1981). This property of Rayleigh-Bénard convection together with relatively simple experimental setup makes the system appropriate for investigation of the transition to turbulence.

The experiments in this work are performed in water $(\operatorname{Pr} \approx 7)$ and at Rayleigh number range from $5 \times 10^{8}$ to $2 \times 10^{9}$. For this parameter space the flow is in the hard turbulence régime. 


\subsection{Turbulence}

Although the meaning of the notion "turbulence" is intuitively clear, there is no generally accepted definition of turbulence. None of the existing definitions are completely satisfactory either because it can not be applied unambiguously in experiments or because it does not coincide with the intuitive physical concept of turbulence. The following are four attempts of the definition of turbulence according to Busse (1981).

The mathematical approach is to separate a laminar from a turbulent flow on the basis of uniqueness of the solutions of the underlying equations. The conductive state in Rayleigh-Bénard convection is unique and when the Ra reaches some critical value more than one solution is possible. The critical values of the governing parameters of a flow can often be analytically determined which makes the method unambiguous. On the other hand, it can not distinguish between the time independent and time dependent flow intuitively connected to turbulence. Thus, the onset of time dependence seems to be a suitable criterion for the definition, which leads to the next attempt. The shortcomings of this approach are that (i) time dependence often occurs in the form of periodic waves, which contradicts aperiodic time dependence one intuitively associates with turbulence; (ii) the transition depends on the history of the particular experiment and can only approximately be predicted on the basis of the external parameters of the system; (iii) the judgment if a flow is time dependent may depend on the frame of reference of the observer. Finally, it is possible to define transition to turbulence as appearance of the first random elements in the time dependence of the system. This definition seems to work well if the random behavior is preceded by a periodic or quasi-periodic time dependence and is in accordance with intuitive perception of turbulence. For instance, Gollub and Swinney (1975) found a fairly distinct onset of broadband noise in the frequency power spectrum preceded by periodic and quasi-periodic states in Couette flow.

Of course, the flow to be dealt with in this work is far from the onset and is turbulent independent of the choice of definition.

Tennekes and Lumley (1982) overcomes the difficulties of precise definition of turbulence by simply listing some of the characteristics of turbulent flows. These characteristics are:

- irregularity - this makes statistics the main approach to turbulence problems,

- diffusivity - which causes rapid mixing and increased rates of momentum, heat and mass transfer; in the Rayleigh-Bénard problem the strong mixing due to turbulence causes the time average of temperature to be constant across the central part of the container whereas the 


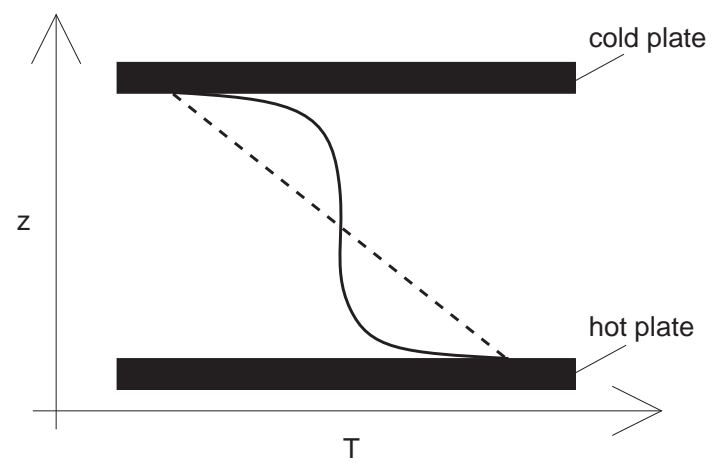

Figure 2.2: Schematic sketch of the temperature profile in the Rayleigh-Bénard convection: linear profile of the conduction state (dashed line) and a profile in a turbulent state (solid line).

total temperature drop is (on average) confined to the narrow thermal boundary layers (Fig. (2.2)),

- large Reynolds numbers (or equivalent control parameter, like in this work the Rayleigh number)

- three-dimensional vorticity fluctuations - an important vorticity-maintenance mechanism known as vortex stretching is absent in two-dimensional flow,

- dissipation,

- continuum - even the smallest scales in a turbulent flow are much larger then molecular scales.

Finally, it should be emphasized that turbulence is a feature of a flow and not of a fluid. That is why most of the dynamics of turbulence is the same independent of the fluid if the control parameters and the boundary and initial conditions are the same.

\subsection{Coherent structures}

Coherent structures can be defined as patterns of motion which can be clearly distinguished from the turbulent background and occur repeatedly in time or space. This quite general definition enables treatment of, for example, small scale vortical structures in the boundary layers as well as one single large scale roll as coherent structures.

Different types of flows produce different patterns, although some of them share certain similarities. In turbulent Rayleigh-Bénard convection the structures can be observed on different length scales. An extensive 


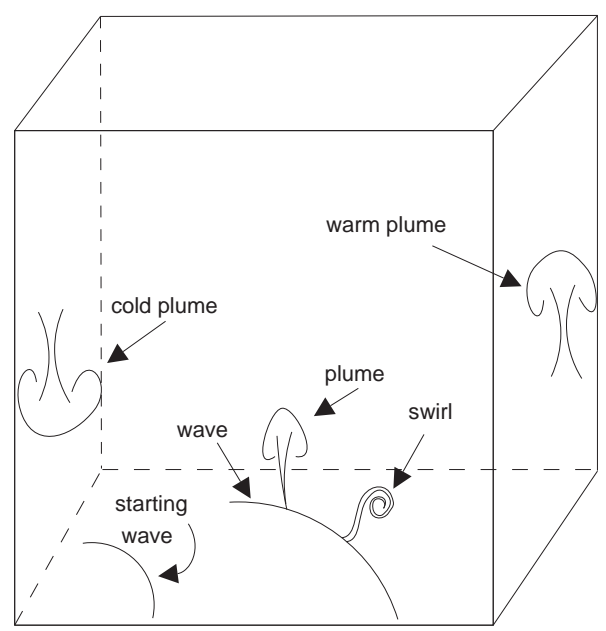

Figure 2.3: Structures in a convection cell according to Zocchi et al. (1990).

study of these structures was done by Zocchi et al. (1990). From direct visualization they obtained the qualitative picture for the hard turbulence régime described briefly in the following. There are two types of structures: those which live in the bulk - the plumes and the spiraling swirls, and the ones living along the boundary layers - the waves. The plumes hit the boundary layers and excite waves; the waves are sustained by the coherent large scale flow, and they further decay to spiraling swirls if inertia dominates over buoyancy, or plumes if buoyancy dominates. The plumes are carried with the large scale flow and excite new waves as they hit the opposite plate (Fig. (2.3)). It should be mentioned here that the plumes can have both (quasi)cylindrical stem as well as a sheet-like one as observed by some researchers (e.g. Funfschilling and Ahlers, 2004).

Another type of coherency is found by Sano et al. (1989). They found coherent oscillation in temperature signal measured near the plates. They also found that this frequency equals the circulation frequency of the large scale flow (which is $U / 4 L$, where $U$ is the velocity of the large scale flow and $L$ is the height of the aspect ratio one container) and that the phase shift of the cross spectrum of two bolometers positioned at the opposite plates is $180^{\circ}$. Recently, Qiu and P.Tong (2002) put this into question and suggested that the coherent frequency equals $U / 2 L$ instead.

The central topic of this thesis is the investigation of coherent structures in turbulent Rayleigh-Bénard convection on different scales and in different regions of the container. 


\section{Chapter 3}

\section{Experimental setup and methods}

$\mathrm{T}^{\mathrm{N}}$ this chapter the general experimental setup is described which is common 1 for all experiments done within the scope of the thesis. The experimental details important for particular experiments are described in the respective chapters.

\subsection{Cells}

The experiments are performed in a cubical and a cylindrical cell.

The cubical cell (Fig. (3.1)) has sides of $20 \mathrm{~cm}$. The sidewalls are made of $1 \mathrm{~cm}$ thick plexyglas. The bottom and the top plates are $1 \mathrm{~cm}$ thick copper plates coated with silver for protection against corrosion. Electrical film heaters $^{1}$ are attached to the bottom plate and it is heated with a constant heat flux. The temperature of the top plate is regulated by circulating water from a thermostat through pipes welded to the top plate. In order to avoid horizontal temperature gradient on the top plate two shorter winding pipes are used rather then one longer. The plate temperatures were uniform in space and time to better then $0.1 \mathrm{~K}$. The top and the bottom plates and the side wall were held together with stainless posts with insulating spacers between the posts and the plates. The walls and the plates are sealed by placing rubber O-rings in between them.

For the experiments in the cylindrical cell a cylindrical inset made of 3 $\mathrm{mm}$ thick plexyglas is put in the cube described above. Everything else (top and bottom plates, temperature regulation, etc.) remains the same. Having the walls with different geometries but exactly the same other conditions makes it possible to investigate the influence of the geometry on the flow.

The whole cell is (except for the visualization experiment) packed in a

\footnotetext{
${ }^{1}$ All technical data about equipment used in the work are given in Appendix A
} 


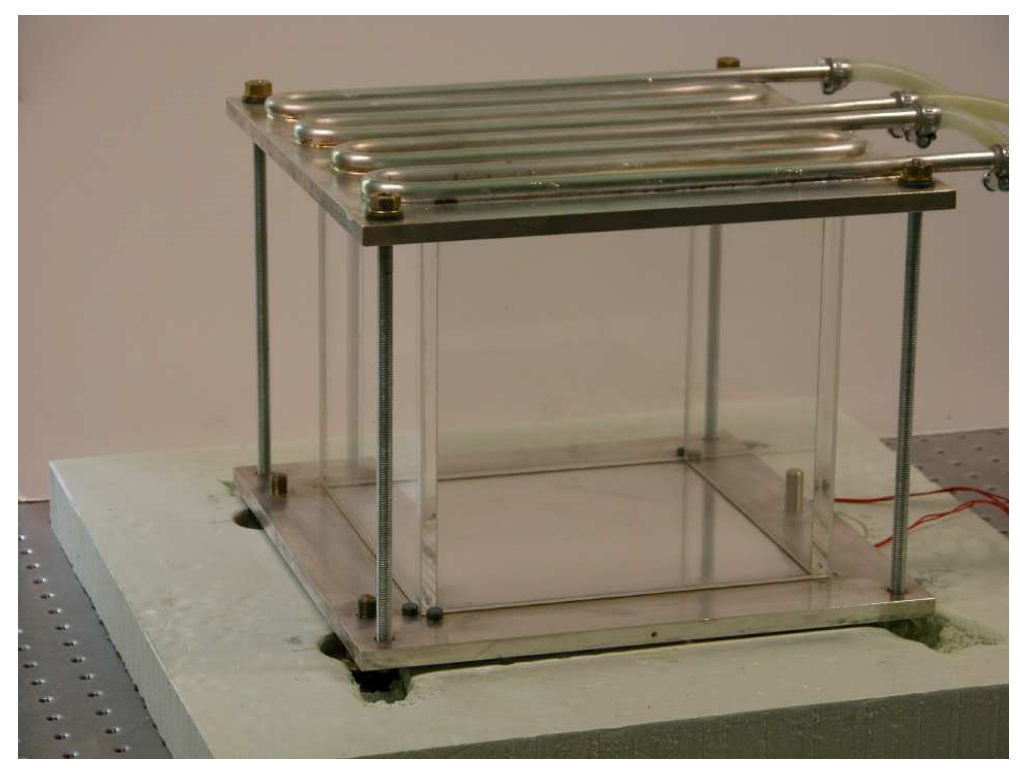

Figure 3.1: Photography of the cubical cell used in the experiments.

styrofoam box with $4 \mathrm{~cm}$ thick walls which insulates the convection cell from the environmental temperature oscillations. Inside the box four ventilators are placed to mix the air around the convection cell. This ensures that the temperature around the sides of the convection cell is the average between the top and the bottom plate temperature, and that there is no periodic vertical oscillation of the air in the surroundings caused by buoyancy.

A $13 \mathrm{~mm}$ travel translation stage with the resolution of $10 \mu \mathrm{m}$ can be mounted above a hole in the center of the top plate. This enables precise vertical positioning of thermistors used in temperature profile measurements and correlation measurements.

The cells were filled with water. Before the water was put into the cell it had always been degassed using a vacuum pump, so that no bubbles could form on the plates in the course of the experiment. In the visualization experiments a $p H$ indicator was dissolved in the water as described in detail in Section 4.1 - this, however, did not change the properties of the fluid.

\subsection{Thermistors and temperature measurement}

Local temperature in the cells is measured with thermistors, electronic components that exhibit a large change in resistance with a change in their body temperature. The ones used in the experiments are of NTC ("Negative Temperature Coefficient") type, i.e. the resistance of a thermistor decreases with the increase of its body temperature. It consists of a thermal probe welded to insulated extension leads and coated with epoxy resin for complete insu- 


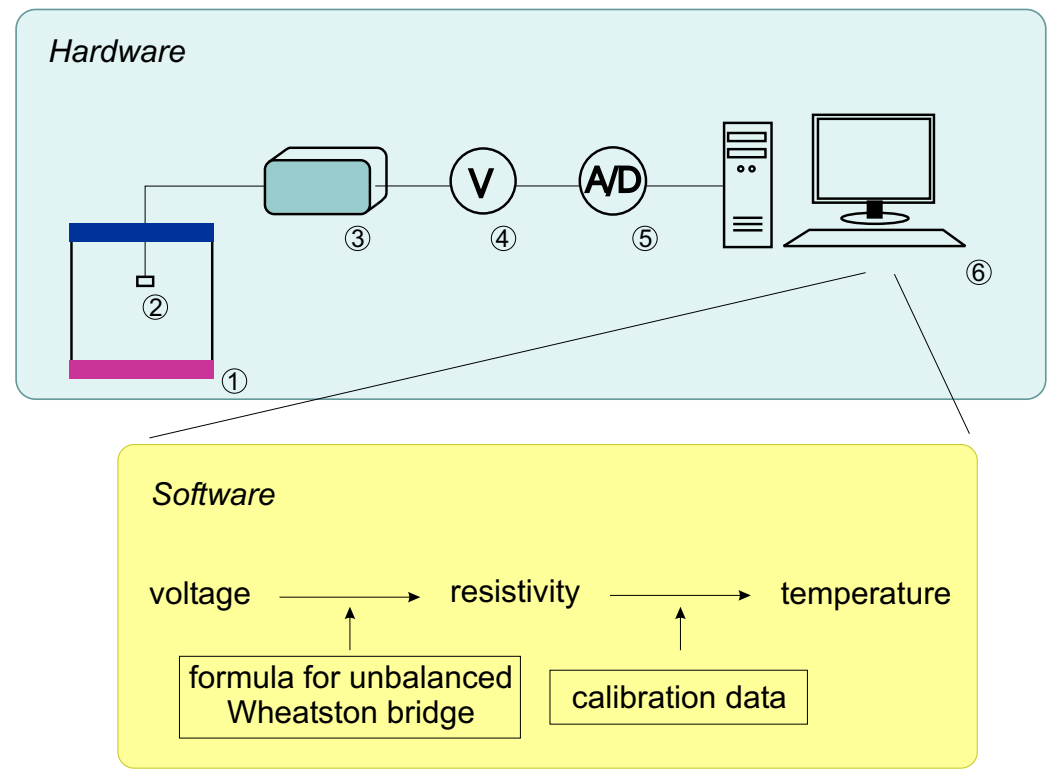

Figure 3.2: Schematic picture of the temperature measurement process in a box filled with water (1). A thermistor (2) is connected to the Wheatston bridge (3) as a resistance arm. As the temperature generally oscillates the bridge is unbalanced, that is the voltage (4) on the bridge oscillates. The voltage is measured and transported to the computer using PCI Bus Data Acquisition Board (5). The time-voltage pairs are saved to the computer disc (6). From the voltage data the resistivity of the thermistor is calculated in each moment and finally from the resistivity the temperature is determined using the calibration data.

lation when immersed in conductive fluids. The thermal time constant of the thermistors is $14 \mathrm{~ms}$. Temperature measurement with a thermistor is an intrusive method and it is of great importance to have a probe which does not significantly change the properties of the flow. The properties of the flow around an obstacle are given by a parameter called "Reynolds number" $\operatorname{Re}=U L / \nu$, where $U$ is the typical velocity of the flow, $L$ is the length scale of the obstacle and $\nu$ is the kinematic viscosity of the fluid. The diameter of the thermistor is $400 \mu \mathrm{m}$, the velocity scale (chosen to be the maximum of time averaged velocity measured vertically from the plate to the center of the cell) is found to be about $7 \mathrm{~mm} \mathrm{~s}^{-1}$ for typical conditions of the experiments (Xin et al., 1996), and the viscosity of water at $20^{\circ} \mathrm{C}$ is $10^{-6} \mathrm{~m}^{2} \mathrm{~s}^{-1}$. All these gives $\operatorname{Re}<3$. Typically, thermistors are positioned in the flow using a tube with a diameter of $1 \mathrm{~mm}$. For this obstacle the Reynolds number is $\operatorname{Re}=7$. For these two Reynolds numbers the flow is known to have two small attached eddies behind the obstacle (Tritton, 1992), but the flow is not disturbed for more then the size of the obstacle in downstream direction so that the flow properties are not significantly influenced by the probes.

All thermistors are calibrated individually against a digital thermometer 
with a $100 \Omega$ platinum sensing element ("Pt100"). Temperature is measured by connecting a thermistor to a Wheatston bridge as a resistance arm. As the temperature generally oscillates the bridge is unbalanced. The voltage on the bridge is then measured and transferred to the computer using a PCI Bus Data Acquisition Board. The board represents a unipolar (that is, always positive) signal as an unsigned 12 bit number, which for the total range of $0-20 \mathrm{mV}$ (a setup typically used in the measurements) gives a resolution of $4.9 \mu \mathrm{V}$. The resistance of the thermistor is then calculated from it. The temperature is determined from the calibration data using spline interpolation between the calibration points ${ }^{2}$. The resolution of the voltage measurements on the bridge of $4.9 \mu \mathrm{V}$ corresponds to a resolution of $0.3 \Omega$ on the thermistor, which finally enables the temperature to be determined with precision better then $10^{-5} \mathrm{~K}$. The measurement process is shown schematically in Fig. (3.2).

\footnotetext{
${ }^{2}$ Software programed for the purposes of the work is listed in Appendix B
} 


\section{Chapter 4}

\section{Coherent structures in the boundary layers}

$\mathrm{T}^{\mathrm{N}}$ this chapter the experiment and the results of the research of the small 1 scale coherent structures are presented. All results are gained from the experiment in the cubical cell described in Section 3.1. The observation in the cylindrical cell and comparison of the phenomenon in the two geometries is postponed to Chapter 6 .

\subsection{Experiment}

The flow in the boundary layers is visualized using the " $p H$ method" (Baker, 1966). The $p H$ indicator "thymol blue" ${ }^{1}$ is dissolved at a concentration of about $10^{-4}$ per weight in the water. The solution is titrated with $\mathrm{HCl}$ and $\mathrm{NaOH}$ just to the acid side of the end point of the indicator so that the solution was orange yellow. If a small electrical potential difference is applied between the upper and lower plates, such that the lower plate is used as cathode, then the $\mathrm{H}^{+}$ions near the lower plate are drawn to that plate where they react to $\mathrm{H}_{2}$ leaving an excess of $\mathrm{OH}^{-}$ions there. This leads to a local increase of the $p H$ value at the lower plate and the solution changes its color to dark blue. Once a darkened fluid particle is carried away from the electrode, it loses through diffusion its $p H$ contrast with the environment and turns orange yellow again. In order to get the layer of dye on the plate as thin as possible the solution must be titrated carefully and the voltage must be chosen as small as possible. The voltage applied between the electrodes in the experiments was typically $1.2-3 \mathrm{~V}$. At higher voltages, the blue dye is separated from the plate and probes regions in which the flow is clearly three dimensional instead of nearly parallel to the boundary.

\footnotetext{
${ }^{1} p H=-\log _{10}\left[\mathrm{H}^{+}\right]$is a measure of the hydrogen ion concentration, $\left[\mathrm{H}^{+}\right]$. A $p H$ indicator is a dye that changes color when $p H$ changes. The "thymol blue" indicator used in the thesis is orange yellow for $p H<9$ and dark blue for $p H \geq 9$.
} 


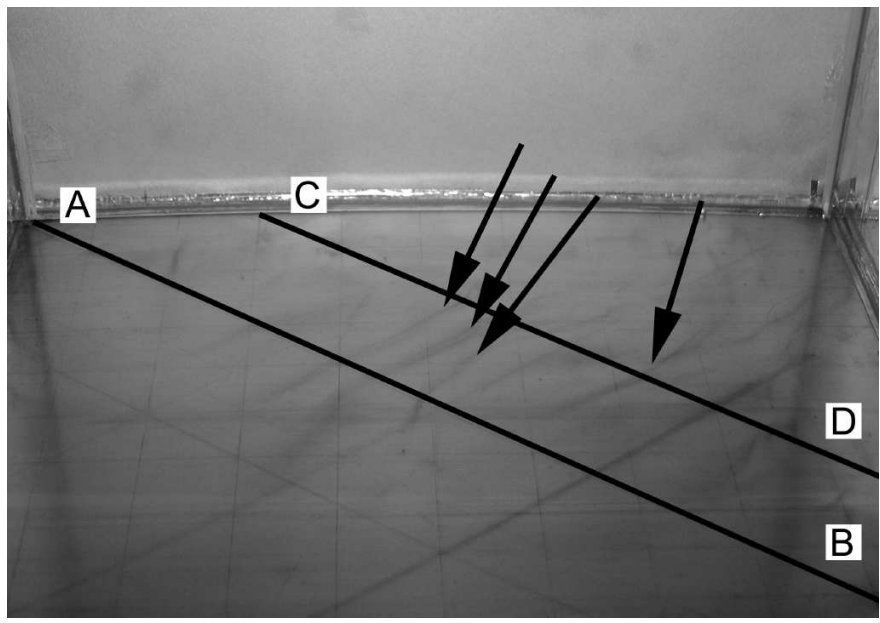

Figure 4.1: View of the bottom plate. The line $\mathrm{AB}$ is the main diagonal of the plate perpendicular to the mean large scale flow (which is from the lower left to the upper right corner. A few streaks are marked with arrows.

If in the thin dyed fluid layer there are regions where the flow converges and, as a consequence, regions where it diverges, then the layer will not stay uniformly dark - the dye will collect in the converging region and the undyed fluid from the bulk will replace the dyed one in the diverging region. Thus the dye marks the areas of convergence which will be shown in the next Sections to form a regular pattern, that is, a coherent structure.

In order to acquire quantitative data, photographs of the bottom plate of the cell were taken with a 5 megapixel CCD camera. One photograph was taken every $60 \mathrm{~s}$ so that consecutive pictures are statistically independent. The camera was operated with software which enables programming of the interval shooting and was connected to the computer via the serial port. A grid of equally spaced lines at intervals of $2 \mathrm{~cm}$ was drawn with a graphite pencil on the plate for calibration of the pictures. The positions at which streaks cross either the main diagonal perpendicular to the mean flow (AB in Fig. (4.1)) or where they cross the diagonal labeled CD in Fig. (4.1) (translated for $2 \sqrt{2} \mathrm{~cm}$ from $\mathrm{AB}$ in downstream direction) is determined manually using a commercial software for digital image editing.

In the coarse of the work a piece of software was developed ("StreakView", see Appendix B) which draws the difference of two pictures pixel by pixel. It was used to make the difference of a picture with streaks and the "background" (picture without the streaks) so that only streaks (in ideal case) are left as the result. That way the streaks are easily identifiable. An example is shown in Fig. (4.2). 


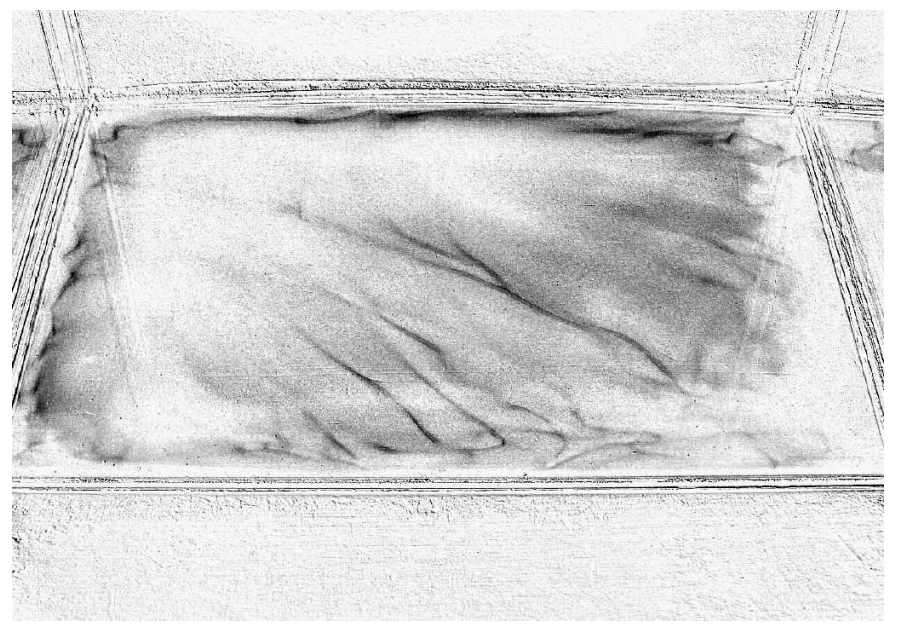

Figure 4.2: Photograph of the lower plates processed with "StreakView" software. The mean large scale flow is from the upper left to the lower right corner.

\subsection{Observation}

The basic observation is that dark fluid accumulates in streaks aligned with the mean wind (Figs. (4.1) and (4.2)). Streaks appear in groups within which there is an apparent periodicity. The streaks are advected by the mean circulation and lift off the plate when they approach a sidewall. The number of visible streaks increases with downstream distance and the first ones appear shortly before they reach the diagonal of the bottom plate perpendicular to the mean flow (the line AB in Fig. (4.1)). Streaks are uniformly distributed over $\mathrm{AB}$ or $\mathrm{CD}$ which indicates that they are not related to possible defects in the plate.

If the voltage between the plates is increased, the thickness of the dyed layer would be increased as described in the previous section. In this case it is possible to observe some properties of the flow on top of the boundary layers. Fig. (4.3) shows such a case. The "waves" as described by Zocchi et al. (1990) (cf. Fig. (2.3)) are clearly identifiable in the upstream part of the flow. Note that they are visible only when the layer of dye is thicker then the boundary layer, which proves the findings of Zocchi et al. (1990) that they live on the interface between the boundary layer and the bulk. The streaks, on the other hand, are visible when the layer of dye is thinner then the boundary layer, which means that they live in the boundary layers.

The experiments were performed for several Rayleigh and Prandtl numbers and across the two diagonals as summarized in Table 4.1 in the first three columns. 


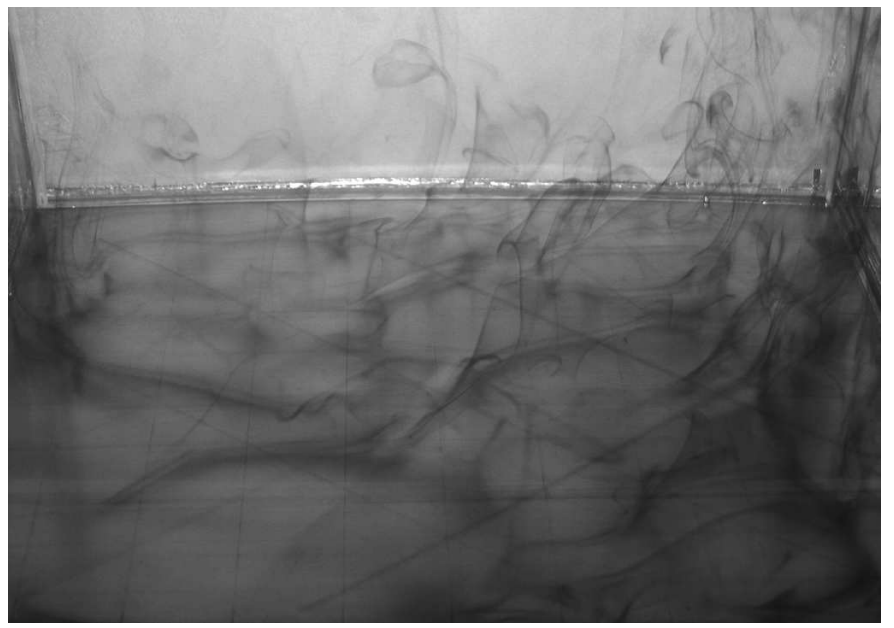

Figure 4.3: Visualization of the flow over the bottom plate, with dye layer thicker then the boundary layers. The large scale mean flow is from the lower left to the upper right corner. The "waves" sketched in Fig. (2.3) are here clearly visible in the upstream part of the flow (circular structures).

\subsection{Mechanism of streak formation}

In order to exactly define the area where the streaks appear it is now necessary to define the thermal boundary layer more exactly. Although several definitions of the thickness of the boundary layer can be found in the literature, the differences in the values obtained in different ways are minor and unimportant for this work. The measurement of the temperature profile with a resolution of $125 \mu \mathrm{m}$ at $\mathrm{Ra}=1.3 \times 10^{9}$ and $\mathrm{Pr}=6.7$ is shown in Fig. (4.4). The thermal boundary layer is then defined as the distance at which the extrapolation of the linear part of the profile equals the mean temperature in the bulk and for this case the thickness is $1.8 \mathrm{~mm}$. For the range of Rayleigh and Prandtl numbers in this work the measured thicknesses of the thermal boundary layer varied by at most for about $10 \%$.

The appearance of streaks indicates that blue dye is swept together by the flow. The convergence of the flow towards the lines where streaks form requires that fluid rises above the streaks and that downwellings exist in between streaks. A pattern of closed streamlines producing a single streak would be a pair of counterrotating vortices superposed on, and aligned with, the mean flow.

Although the streak distribution is uniform over the plate in the direction perpendicular to the flow, the histogram of the streak separations has a pronounced peak. The bar charts in Fig. (4.5) are examples of the distributions for $\mathrm{Ra}=1.3 \times 10^{9}$ and $\mathrm{Pr}=6.7$ across the line $\mathrm{CD}$ (lefthand picture) and across the main diagonal $\mathrm{AB}$ (righthand picture). The peaks have values between $6 \mathrm{~mm}$ and $10 \mathrm{~mm}$ for the range of parameters inves- 


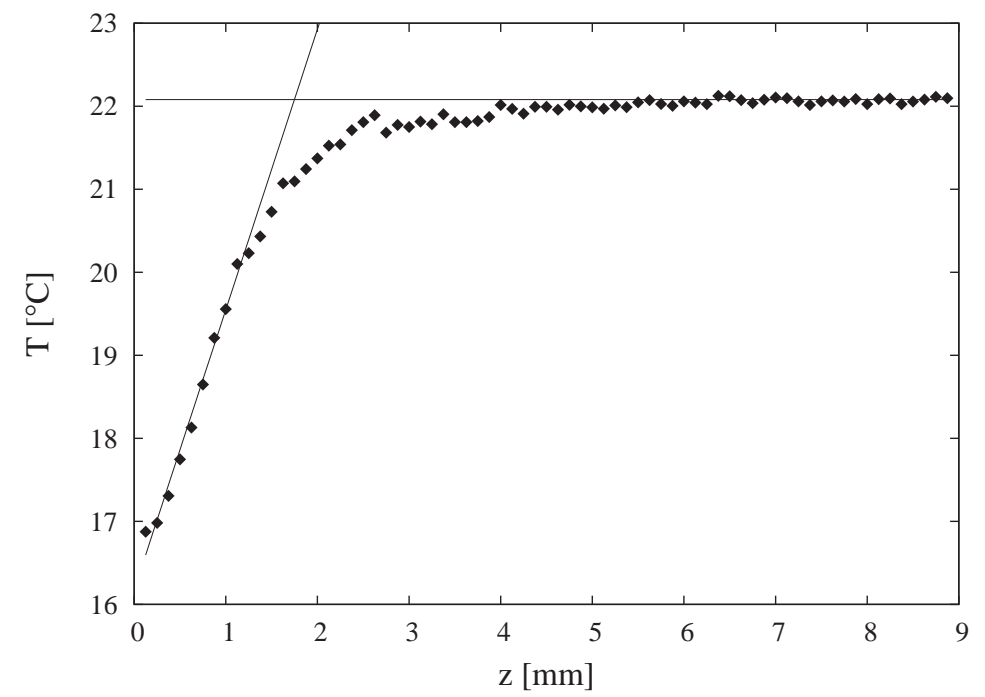

Figure 4.4: The mean temperature as function of distance $\mathrm{z}$ from the top plate for $\mathrm{Ra}=1.3 \cdot 10^{9}$ and $\operatorname{Pr}=6.7$. The intersection of the solid lines defines the thickness of the boundary layer (see text).

tigated. Furthermore, the distribution towards larger distances seems to have an exponential tail. These two features - the pronounced peak and the exponential tail - give rise to two approaches to the explanation of the distribution. On one hand the peak can be interpreted as a hint at an underlying periodicity. This idea leads to linear stability analysis and solution of the perturbed equation in terms of normal modes. On the other hand, the exponential distribution is typical for many stochastical processes in nature and motivates the explanation in terms of statistics. Both of these approaches are presented in the next two sections.

\subsubsection{Linear stability analysis}

The thermal boundary layer itself is stable with respect to convection within that layer as can easily be proved by comparing the actual Rayleigh number with the critical one for the onset of convection. The critical Rayleigh number for rigid-free boundaries is 1000 (Chandrasekhar, 1981) and with the imposed horizontal flow on the upper boundary (due to the large scale mean flow) it becomes even higher. The actual Rayleigh number of the boundary layer in the experiment is $\mathrm{Ra} \approx 560$, that is, below the critical value for the onset of convection. However, the hot fluid of the boundary layer at the bottom plate underneath the cold bulk is Rayleigh-Taylor unstable (as well as, of course, the cold boundary layer on top of the hotter bulk at the upper plate). In this section the linear stability analysis of the system "boundary layer - bulk" is carried out taking into account the shear flow caused by the 

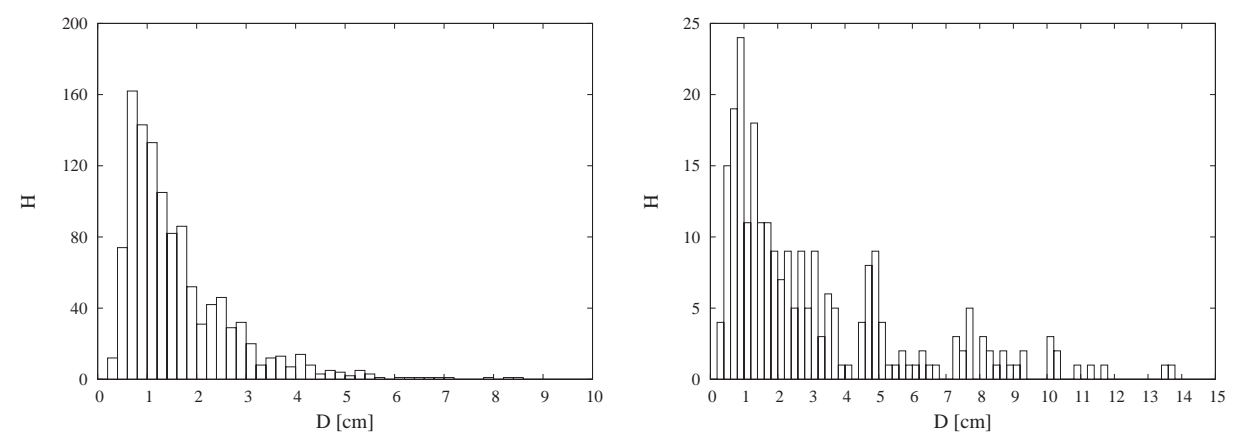

Figure 4.5: Two experimental histograms of streak separations. Lefthand figure shows the separations across the diagonal CD and is based on 134 photographs showing a total of 1277 streaks. The righthand figure shows the histogram for the main diagonal $\mathrm{AB}$ and is based on 56 pictures showing the total of 305 streaks.

large scale wind. The model used in the calculation is shown in Fig. (4.6).

Let the basic state be defined by the local time averaged values of the velocity and temperature, $\vec{U}(z)$ and $T_{0}(z)$, respectively, and let this state be slightly perturbed, so that

$$
\begin{array}{r}
T(x, y, z)=T_{0}(z)+T^{\prime}(x, y, z) \\
\vec{u}(x, y, z)=\vec{U}(z)+\vec{v}(x, y, z)
\end{array}
$$

where $T(x, y, z)$ and $\vec{u}(x, y, z)$ are total temperature and velocity at $(x, y, z)$ respectively, and $T^{\prime}(x, y, z)$ and $\vec{v}(x, y, z)$ are temperature and velocity perturbations, respectively. The $x$ axes is chosen to be in the direction of the large scale mean flow $\vec{U}(z)$, that is, parallel to the streaks as shown in Fig. (4.1). The linear stability problem is described by the following equations:

$$
\begin{gathered}
\partial_{t} \vec{v}+(\vec{v} \cdot \nabla) \vec{U}+(\vec{U} \cdot \nabla) \vec{v}=-\frac{1}{\rho} \nabla p+\nu \nabla^{2} \vec{v}-\vec{g} \alpha T \\
\nabla \cdot \vec{v}=0, \\
\partial_{t} T+(\vec{v} \cdot \nabla) T_{0}+(\vec{U} \cdot \nabla) T=\kappa \nabla^{2} T .
\end{gathered}
$$

These equations can be nondimensionalized using the total thickness of the investigated layer $z_{\max }$ as length scale, the maximal averaged velocity $U\left(z_{\max }\right)$ as velocity scale and the temperature difference between the plate and $z_{\max }$ as temperature scale. Taking $\hat{k} \cdot \nabla \times$ and $\hat{k} \cdot \nabla \times \nabla \times$ of the nondimensional momentum equation gives together with the nondimensional heat equation the following system:

$$
\begin{gathered}
\left(\partial_{t}+\vec{U}(z) \partial_{x}\right) \eta+\frac{d U(z)}{d y} \partial_{y} w=\operatorname{Re}^{-1} \nabla^{2} \eta \\
\left(\partial_{t}+\vec{U}(z) \partial_{x}\right) \nabla^{2} w-\frac{d^{2} U(z)}{d z^{2}} \partial_{x} w-\operatorname{Re}^{-1} \nabla^{2} \nabla^{2} w=\operatorname{RaRe}^{-2} \operatorname{Pr}^{-1} \nabla_{h}^{2} T^{\prime} \\
\left(\partial_{t}+\vec{U}(z) \partial_{x}\right) T^{\prime}+w \frac{d T_{0}(z)}{d z}=\operatorname{Re}^{-1} \operatorname{Pr}^{-1} \nabla^{2} T^{\prime}
\end{gathered}
$$




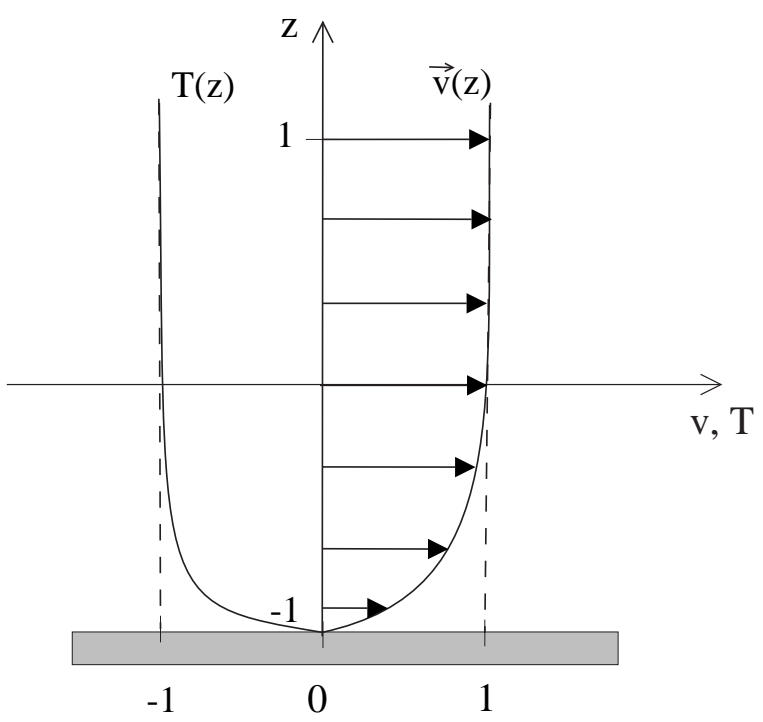

Figure 4.6: Model used for the calculation of the most unstable mode using linear stability analysis. The velocity is scaled with its maximal value, temperature with the temperature difference between the value at the top of the computational domain and the value at the plate and the length is scaled with the half of the height of the computational domain.

where $\eta \equiv \hat{k} \cdot \nabla \times \vec{v}$ is the vertical component of the vorticity, $w \equiv \vec{v} \cdot \hat{k}$ is the vertical velocity component, $\nabla_{h}^{2} \equiv \partial_{x}^{2}+\partial_{y}^{2}$ and $\operatorname{Re}=\frac{U L}{\nu}$ is the nondimensional Reynolds number. The solution is searched in terms of normal modes, so that the dependence of $\vec{v}$ and $T$ on $x, y$, and $t$ is supposed to be of the form $e^{\sigma t} e^{i\left(k_{x} x+k_{y} y\right)}$. For any fixed wave numbers $k_{x}$ and $k_{y}$ the equations (4.6)-(4.8) pose an eigenvalue problem with the growth rate $\sigma$ as eigenvalue. The boundary conditions used to complete the eigenvalue problem are $\vec{v}=0$ and $T=0$ at $z=0$ and $z=z_{\max }$. The eigenvalues are computed numerically with a Chebychev spectral method.

In order to check the influence of the large scale mean flow velocity on the wavelength of the most unstable mode the result of the calculation with real temperature and velocity profiles taken from Tilgner et al. (1993) is compared with the calculation with the same temperature profile and the mean large scale velocity set to zero ${ }^{2}$. The Rayleigh and Prandtl numbers in this experiment were $\mathrm{Ra}=1.1 \cdot 10^{9}$ and $\mathrm{Pr}=6.6$. The most unstable mode turns out to be unaffected by the large scale wind - velocity is apparently

\footnotetext{
${ }^{2}$ The temperature profile in the paper and in the thesis were actually measured at the upper plate and the model is tailored for the lower plate, but as the system is vertically symmetric it is trivial to transform the profile so that it can be used with the model, or to transform the model for use with the profile at the upper plate. In the thesis the former was done.
} 


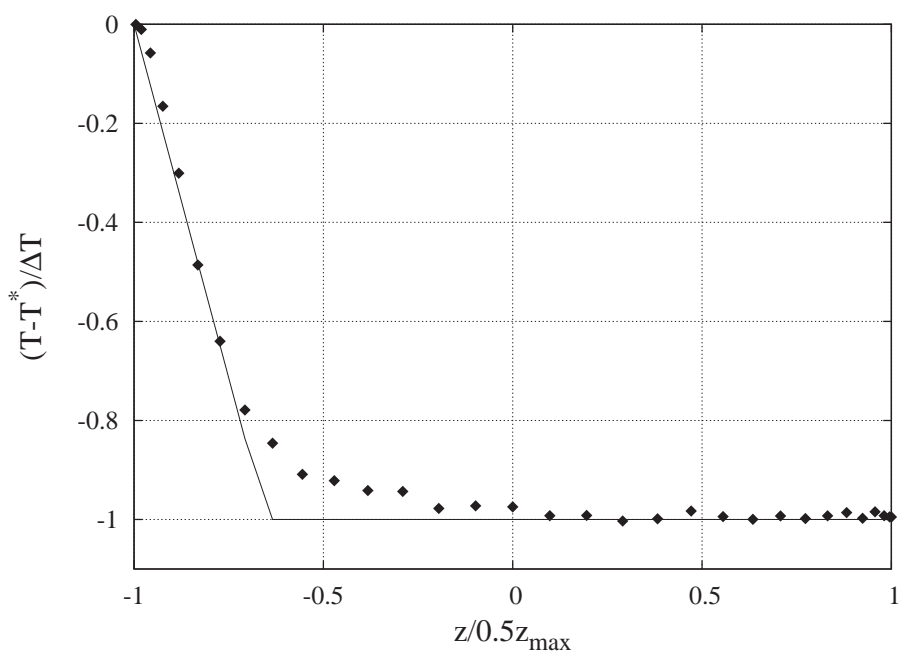

Figure 4.7: Measured temperature profile for $\operatorname{Ra}=1.3 \cdot 10^{9}, \operatorname{Pr}=6.6$ (diamonds) and aproximation used for some computations (solid line). Temperature and length are nondimensionaled (see Fig.(4.6)). $T^{*}$ is the temperature at the plate.

too small to play a significant role except that it gives a preference to longitudinal over transverse vortices. This result makes it possible to perform the calculations using the real temperature profile measured in the cubical cell described in Section 3.1 (the profile is shown in Fig. (4.4)) despite the fact that the velocity was not measured at all in the framework of the thesis.

Next, the dependence of the wavelength of the most unstable mode $(\lambda)$ on the total thickness of the examined layer $\left(z_{\max }\right)$ was investigated. A simple approximation shown in Fig. (4.7) was used for the temperature profile and Rayleigh and Prandtl numbers were $\mathrm{Ra}=1.3 \cdot 10^{9}$ and $\operatorname{Pr}=6.8$. The result is presented in Fig. (4.8). For $z_{\max }>8 \mathrm{~mm}$ the wavelength $\lambda$ saturates to the value $\approx 8.4 \mathrm{~mm}$. This result makes it possible to compute $\lambda$ using as total depth $z_{\max }$ any value greater then $8 \mathrm{~mm}$.

Now the wavelength $\lambda$ and the corresponding growth rate $\sigma$ can be computed for the real temperature profile shown in Fig. (4.4) (see footnote ${ }^{2}$ ) and $z_{\max }=20 \mathrm{~mm}$. The wavelength of the most unstable mode turns out to be $\lambda=8.8 \mathrm{~mm}$ and the growth rate $\sigma=0.6 \mathrm{~s}^{-1}$. The computed $\lambda$ is in very good agreement with the measurements - the histogram of the streak separations has the peak at about that value (Fig. (4.5)). On the other hand, the computed growth rate combined with the advection velocity implies that there should be at least seven times as many visible streaks on the diagonal CD than on AB (Fig. (4.1)). In reality, this factor is approximately 1.5.

The fact that the wavelength of the structures calculated under assumption that they originate from Rayleigh-Taylor instability agrees with the measurement, but the growth rate is much larger then measured, can be explained as follows. If the structures are visualized far away from the place 


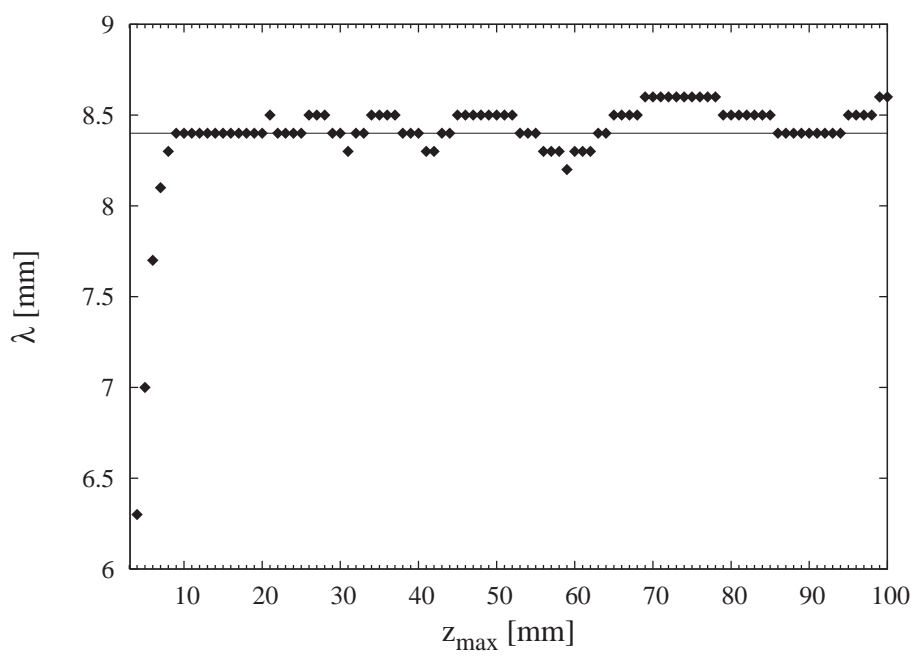

Figure 4.8: The value of the wavelength of the most unstable mode (diamonds) becomes independent of the value of $z_{\max }$ and saturates to $8.4 \mathrm{~mm}$ (solid line).

they originate, then the linearized equations do not model the phenomenon adequately, because the nonlinear term in the Navier-Stokes equation starts to play an important role by limiting further exponential growth. That is, the observed growth rate is smaller then the calculated because the observation is done in the régime beyond the exponential growth.

From the results in this Section it can be concluded that Rayleigh-Taylor instability is a reasonable explanation for the streak formation, but the visualization was done too far away from the position where the disturbances originate to be modeled completely by linearized equations.

In the next section another approach is employed to explain the observed small scale coherent structures, based on the assumption that they are formed by a stochastic process.

\subsubsection{Stochastic process}

In the previous attempt to explain the mechanism of the streak formation, the peak in the histogram of separations was interpreted as the result of periodicity. Alternatively, the distribution of the streak separations and the distribution of the number of streaks in any moment in time (Fig. (4.11)) can be explained in terms of a stochastic process as follows.

Consider a diagonal (AB or $\mathrm{CD}$ on Fig. (4.1)) of length $L$ and denote by $x$ the distance along the diagonal from one of its end points (Fig. (4.9)). For simplicity, it will be assumed that all structures have the same lateral size $\delta$. If a streak at location $x$ is created by a pair of vortices, the streak is surrounded by a vortex of size $\delta / 2$ on each side and the structure extends from $x-\delta / 2$ to $x+\delta / 2$. The point at $x-\delta / 2$ will be called the "left edge" 


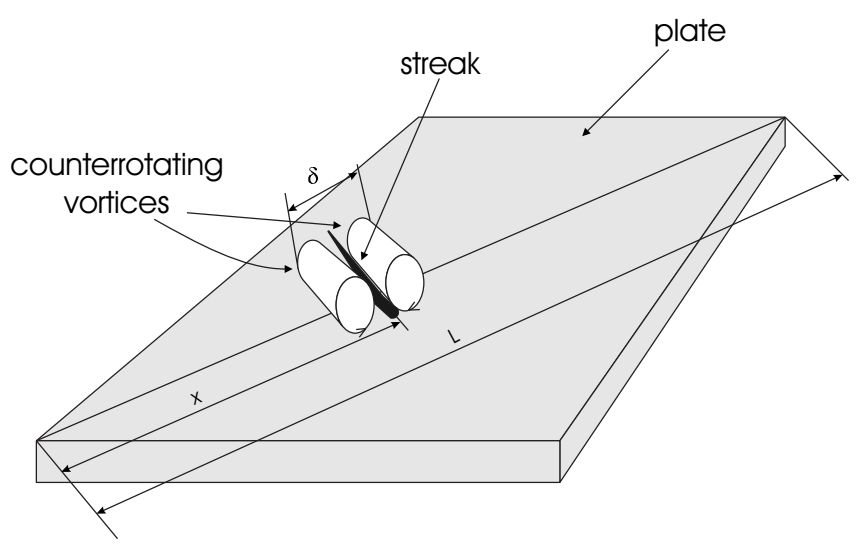

Figure 4.9: Geometry of the problem described in the text.

of the coherent structure.

Denote by $p \mathrm{~d} x$ the probability to find a left edge in an interval $\mathrm{d} x$. The probability density $p$ is independent of $x$ as justified by the uniform distribution of the streaks along the diagonal observed experimentally. The probability to find a segment of length $l$ free of left edges is then $e^{-p l}$. In order to compute the experimental histogram, the finite length of the diagonal must be taken into account. If two streaks are separated by a distance $D$, the leftmost of the two streaks must have a left edge at a distance of at most $(L-D-\delta)$ from the left end of the diagonal so that the second streak still fits onto the diagonal. Left edges are equally distributed in the interval $[0, L-\delta]$. The probability to find two streaks separated by a distance between $D$ and $D+\mathrm{d} D$ is equal to the probability that a streak picked at random is in the interval $[0, L-D-\delta]$, multiplied by the probability not to find a left edge over a distance $(D-\delta)$, multiplied by the probability to find one in the following interval $\mathrm{d} D$, which is all taken together equal to

$$
\frac{L-D-\delta}{L-\delta} e^{-p(D-\delta)} p \mathrm{~d} D .
$$

To be able to compare the experimental histogram constructed with the bin size $\Delta D$ with the theoretical one, in the probability given above $\mathrm{d} D$ must be replaced with $\Delta D$ and the whole expression must be multiplied with the total number of streaks $N_{p} N$ observed, where $N_{p}$ is the number of photographs used in the evaluation and $N$ is the average number of streaks per photograph. The theoretical histogram of streak separations then reads:

$$
H(D)= \begin{cases}\frac{L-D-\delta}{L-\delta} p e^{-p(D-\delta)} \Delta D N_{p} N & , \delta<D<L-\delta \\ 0 & , \text { otherwise }\end{cases}
$$

This expression is used to fit the histogram of streak separations like the ones in Fig.(4.5) 
Another quantity to be found is the probability distribution for the number of streaks in any one photograph (that is, at any moment). Let $P(n, l)$ be the probability to find $n$ streaks at any given time in an interval of length l. $P(n, l)$ can be computed recursively as follows. The probability not to find a streak in the interval of total length $l$ is

$$
P(0, l)= \begin{cases}1 & , l<\delta \\ e^{-p(l-\delta)} & , l \geq \delta .\end{cases}
$$

The probability to find $n$ left edges in the interval $l$ is the probability not to find a left edge of a structure up to location $x$, multiplied by the probability to find one immediately afterwards, multiplied by the probability to find $(n-1)$ further streaks in the remaining interval, integrated over all $x$ in which the left edge of a structure can be located:

$$
P(n, l)= \begin{cases}0 & , l<n \delta \\ \int_{0}^{l-\delta} p e^{-p x} P(n-1, l-x-\delta) d x & , l \geq n \delta .\end{cases}
$$

The parameter $\delta$ is now chosen to have the value at which the experimental histogram of separations has the maximum and $p$ is found so that $P(n, l)$ fits the experimental histogram of separations as well as possible. The fact that $\delta$ - the size of the whole structure needed to produce a streak - is chosen to be a constant implies the assumption that all vortices have the same size. This is probably not a realistic assumption and leads to an error in the prediction of separation for very small distances as will be explained later. The $p$ found this way is then plugged into the expression (4.9) for $H(D)$ and the fit $H(D)$ to the experimental histogram of the number of streaks per picture can now be verified. The results of this procedure are shown in Figures (4.10) and (4.11). It can be seen that the experimental data can be very well fitted by the model without the assumption of periodicity in the streak separations.

The assumption that $\delta$ is equal for all structures is the reason that $H(D)$ equals zero for $D<\delta$ which obviously poorly reproduces the histogram for small $D$. In order to better reproduce the observed data in this region, a more complex model using a distribution of $\delta$ instead of its constant value could be employed. On the other hand, that procedure would introduce more adjustable parameters, but would not contribute much to illuminating the phenomenon, so that the accuracy of the model for small $D$ is sacrificed in favor of simplicity.

Values of $\delta$ defined as the distance at which the separation distribution has the peak are determined for different values of Rayleigh and Prandtl numbers reached in the experiments with a goal to determine a dependence of $\delta$ on the parameters. The experimental histograms are constructed with bin size $2 \mathrm{~mm}$, which slightly influences the determination of $\delta$, nevertheless 


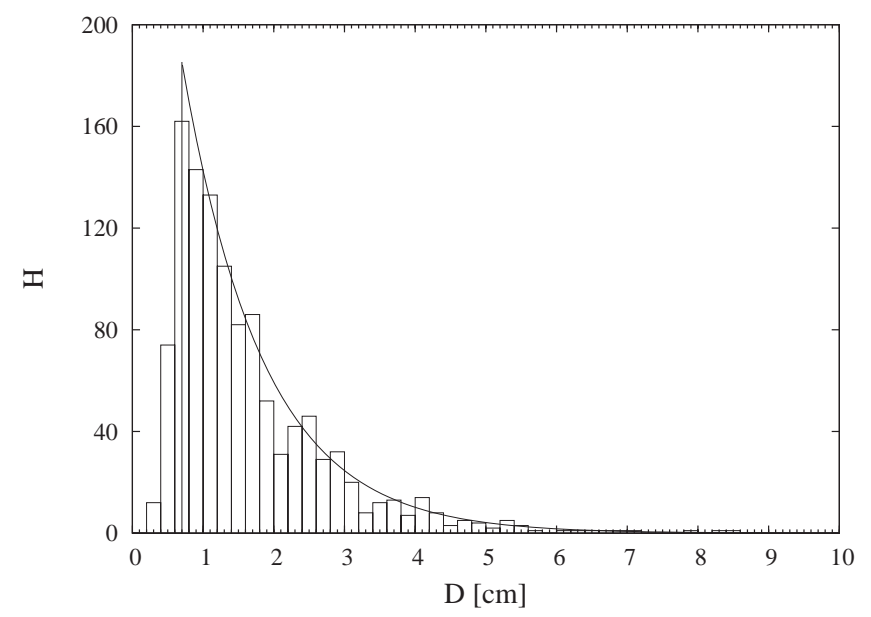

Figure 4.10: The bar chart is a histogram of the streak separation on the line CD in Fig. (4.1), and the solid line is the theoretical curve given by Eq. (4.9) (for $\Delta D=0.2 \mathrm{~mm}, \delta=6 \mathrm{~mm}$ and $p=0.82)$.

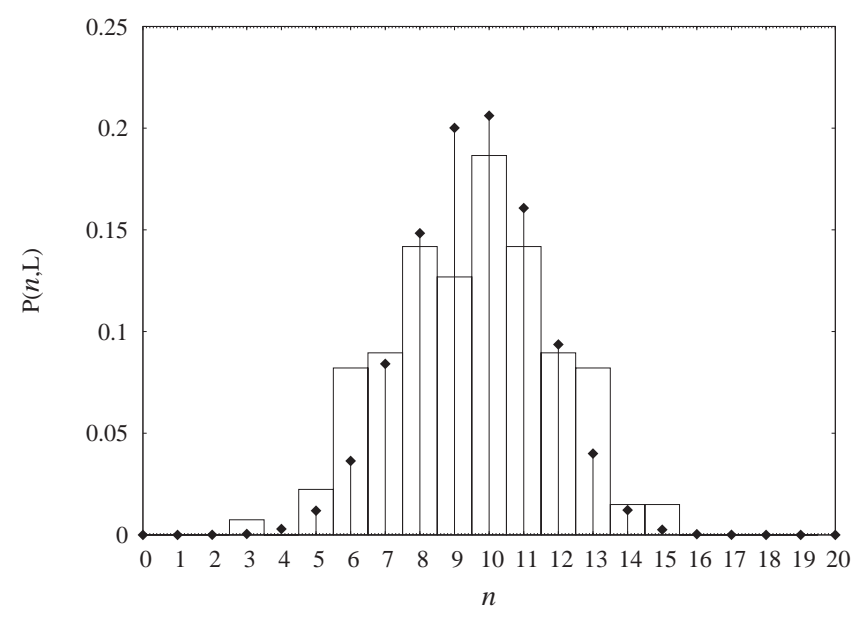

Figure 4.11: The bar chart is the probability distribution for the number of streaks $n$ visible in any one photograph on the line CD for the same data as in Fig. (4.10). The diamonds are $P(n, L)$ according to Eq. (4.10) and (4.11) for $L=198 \mathrm{~mm}$, $\delta=6 \mathrm{~mm}$ and $p=0.82$. 
Table 4.1: Minimal streak separation $\delta$ determined for different Ra and Pr according to the procedure described in the text.

\begin{tabular}{llll}
\hline Ra & Pr & $\begin{array}{l}\text { Position } \\
\text { (see Fig. 4.1) }\end{array}$ & $\begin{array}{l}\delta \\
(\mathrm{mm})\end{array}$ \\
\hline $5.0 \times 10^{8}$ & 6.5 & $\mathrm{CD}$ & 10 \\
$1.3 \times 10^{9}$ & 6.7 & $\mathrm{CD}$ & 6 \\
$1.3 \times 10^{9}$ & 6.7 & $\mathrm{AB}$ & 8 \\
$1.4 \times 10^{9}$ & 3.6 & $\mathrm{CD}$ & 8 \\
$2.0 \times 10^{9}$ & 6.2 & $\mathrm{CD}$ & 10 \\
\hline
\end{tabular}

no evident tendency in change of $\delta$ could be found for the modest range of parameters in the experiments. The result is presented in Table 4.1. Of course, it is not to be expected that $\delta$ does not scale with Ra and/or Pr, but the range of parameters that could be reached in this work was too small to notice it.

\subsubsection{Comparison with streaks in isothermal shear flows}

Periodic streamwise vortices are well known from isothermal momentum boundary layers where they have periodicity length of about 100 wall units (Smith and Metzler, 1983), where one "wall unit" is $\nu^{1 / 2}(d U / d z)^{-1 / 2}, \nu$ is the viscosity and $d U / d z$ the velocity gradient at the wall. Hence, the expected peak of the streak separations for the cell at hand would be 85 $\mathrm{mm}$, which is a distance ten times bigger then actually measured. This is not surprising, as the periodicity of 100 wall units occur at much higher Reynolds numbers then in the convection cell where the Reynolds number based on the thickness of the viscous boundary layer and the velocity at the edge of the boundary layer is less then 30 .

Transient energy growth is a theory that successfully explains the streamwise vortices in isothermal boundary layers. The linear stability analysis gives critical parameters for exponential growth of an initial perturbation and energy methods give conditions for no energy growth. In the case where the flow parameters are smaller then critical for exponential growth but higher then critical for energy growth the transient energy growth can occur for moderate time $t>0$ although the perturbation eventually decays for $t \rightarrow \infty$. The reason is the nonorthogonality of the linearized stability problem, which can cause e.g. the transient growth as large as $10^{10}$ times the energy of the initial perturbation in a viscous channel flow (Reddy and Henningson, 1993). In order to find out if this mechanism causes also the formation of the streaks in turbulent Rayleigh-Bénard convection the calculations of Reddy and Henningson (1993) and Butler and Farell (1992) were extended by inclusion of a linear temperature profile and the buoyancy 


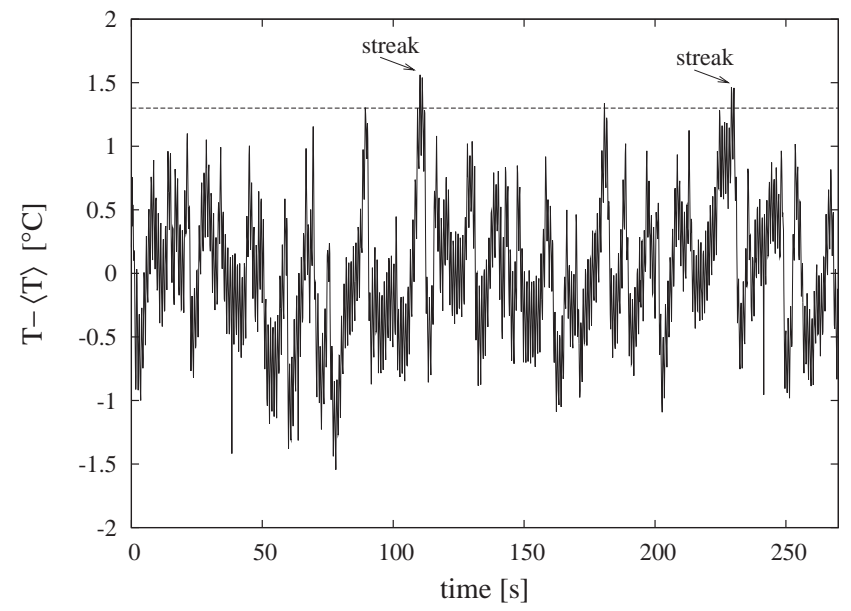

Figure 4.12: Temperature signatures of streaks measured at the distance of about $1 / 8$ of the thermal boundary layer thickness from the plate.

force. However, at the parameters relevant for the experiment, no significant transient amplifications occurs, so that this mechanism must be excluded.

\subsection{Outlook}

Further experiments over a larger span of Rayleigh and Prandtl numbers would be helpful to gain more information about the streaks in the boundary layers of the turbulent thermal convection. The visualization method presented in Section 4.1 is possible only in water. In order to achieve other values of the parameters it is necessary to use different media so it is helpful to develop other methods for the streak detection which would not be dependent on a specific fluid.

Knowing the relative temperature of a streak compared to the surrounding fluid would enable detection of the streaks in any fluid where the temperature can be measured in the boundary layers. With this motivation in mind a less extensive study of the temperature signature of a streak was performed. The measurements were done as follows. The thermistor was fixed at a point in the boundary layer and the temperature was measured for a certain period of time. Times at which a streak hits the thermistor and when it leaves it were noted. The temperature signal is then checked at that times. A representative result is shown in Fig. (4.12).

Analyzing several pictures like Fig (4.12), an approximate criterion for determining a streak based only on temperature signal can be established. This criterion is:

(the maximal temperature in the streak) $>\langle T\rangle+b \times \operatorname{rms}(\mathrm{T})$ on the hot plate, 
or

(the minimal temperature in the streak) $\langle\langle T\rangle-b \times \operatorname{rms}(\mathrm{T})$ on the cold plate,

where $\operatorname{rms}(\mathrm{T})$ is the local rms of the temperature, $\langle T\rangle$ the local mean temperature, and $b$ the factor of magnitude of about 2.5. In Fig. (4.12) the threshold value of $\operatorname{rms}(\mathrm{T})$ of $1.3^{\circ} \mathrm{C}$ corresponds to $b=2.5$.

A similar approach is based on velocity measurements. As a pair of counterrotating vortices push the fluid, which was originally near the plate, in between them the resulting streak must have lower velocity then the surroundings. Thus, by measuring momentary horizontal velocity field in the boundary layers the stripes of low velocity fluid are the streaks described in this Chapter. 


\title{
Chapter 5
}

\section{Coherent structures in the bulk}

\begin{abstract}
distinct feature of turbulent Rayleigh-Bénard convection in a closed A container is the coherent large scale mean flow (by different researchers also called "wind" or "large scale circulation" - LSC) which spans the height of the container and has a single cellular structure. It was discovered by Krishnamurti and Howard (1981) using flow visualization and many of its features are still extensively studied, for instance its sustaining mechanism, the magnitude and the direction of the velocity field or influence on the heat transport. In this chapter the large scale circulation is studied mainly by means of temperature measurements at different positions in the cell.

Additionally, on the length scales between the largest scale in the flow and the small dissipation scales two prominent length scales are discovered and their origin explained. The fact that only two length scales dominate the flow in the bulk contradicts the classical idea of fully developed turbulence in which eddies of all sizes are present in the flow and energy cascades from larger eddies to eddies of the next smaller size. Furthermore, measuring the spatial correlation and time power spectra the validity of Taylor hypothesis could be tested. Before the sections in which the large scale circulation and structures on intermediate scales are studied the experimental setup is described and some important length and time scales in the flow are calculated.
\end{abstract}

\subsection{Experimental setup}

The experiments are performed in the cubical cell with thermistors described in Chapter 3. For the fine resolution temperature measurement used to resolve the thermal boundary layer the following setup was used. The translation table was fixed on the plexyglas plate which is fixed on top of the container's upper copper plate. It is positioned so that the thermistor can 


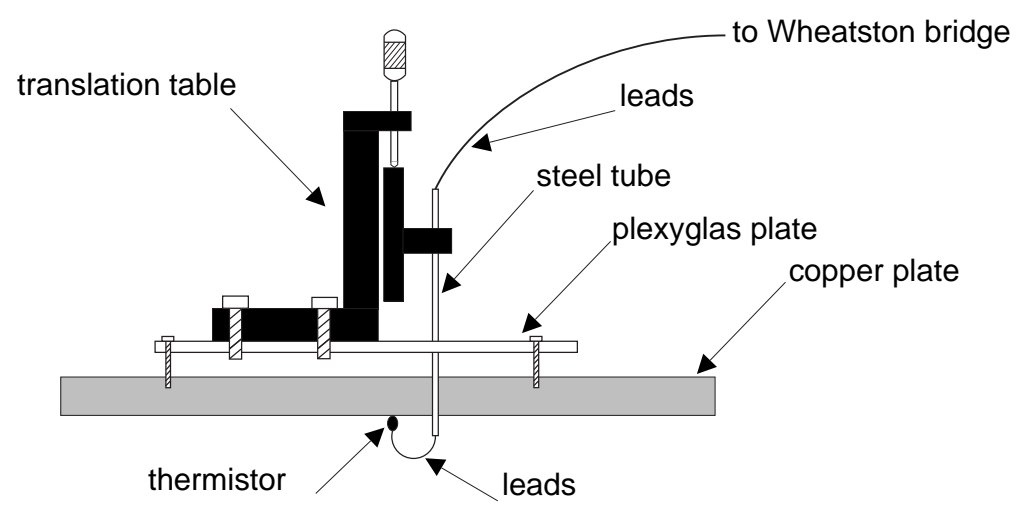

Figure 5.1: Schematic view of the upper plate with a thermistor hold by the translation table.

be lowered through a hole in the middle of the plate. The thermistor leads are drawn through a stainless steal tube with the outer diameter $0.8 \mathrm{~mm}$ and $120 \mathrm{~mm}$ long. The tube is then fixed in the translation table holder. On the thermistor side the leads protrude for about $15 \mathrm{~mm}$ out from the tube and are bent in the $U$ shape so that the thermistor can touch the plate when lowered into the cell (Fig. (5.1)).

Simultaneous measurements along the vertical axes of the cell were done with a thermistor array consisting of nine thermistors evenly spaced $20 \mathrm{~mm}$ one from another, the first one being $20 \mathrm{~mm}$ from the upper plate. The thermistors were attached to a steel tube so that the leads were drawn between the tube and the rubber coating around the tube. The leads on the thermistor side were bent perpendicular to the tube so that the thermistors were about $15 \mathrm{~mm}$ away from the tube. This is a compromise between the wish to measure exactly in the middle of the cell and the need to measure as far away from the holder as possible to avoid the influence of the holder on the flow at the place where the temperature was measured. The leads were drawn through the tube which was hold in place by a holder above the hole in the middle of the upper plate.

The sampling frequency was typically $4.4 \mathrm{~Hz}$ which ensured that the power spectra of the temperature oscillations cover the whole range of frequencies up to (or very near to) the dissipation range. Additionally, it ensures that the $50 \mathrm{~Hz}$ disturbance from the electrical network (and some other disturbances) are aliased to the high frequency end of the spectra, so that they do not influence the analysis.

The flow visualization is performed using thermochromic liquid crystals - a chemical agent which when dissolved in water react to changes in temperature by changing color. Using the crystals it is possible to visualize both velocity and temperature instantaneous fields in the cell and to some extent test the theoretical assumptions. 


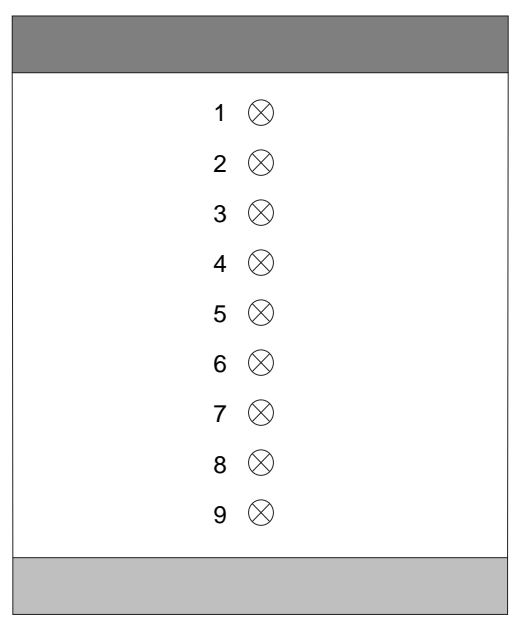

Figure 5.2: Positions of thermistors used for simultaneous temperature measurements and their labels used in text. Thermistors are at distances $20 \mathrm{~mm}$ one from another, thermistors 1 and 9 are at $20 \mathrm{~mm}$ from the upper and lower plates, respectively.

\subsection{Some important scales in turbulent convection}

One of the characteristics of turbulent convection is the existence of several specific length scales, some of which assume specific roles in the description and analysis of the flow. Before proceeding to the determination of the coherent structures in the bulk, a few important length and time scales are calculated in this Section. These are: the largest length scale, the length scales at which the energy and the temperature variance are dissipated, Bolgiano length, diffusion time, turnover time and the time scale which separates the inertial and the dissipation range.

The energy is supplied to the flow through the temperature difference over the distance between the plates. This distance (that is, the height of the cell, $L$ ) is the largest length scale in the flow.

Given the mean velocity of the large scale flow in the cell, a time scale of one turnover can be determined. The mean velocity is $U=9.8 \mathrm{~mm} \mathrm{~s}^{-1}$ (see Section 5.3.1), so that the turnover time is $4 L / U \approx 80 \mathrm{~s}$.

The kinetic energy is dissipated by the viscosity on the so called "Kolmogorov length", $\eta$. Since the small scale motions tend to have small time scales, one can assume that these motions are statistically independent of the relatively slow large scale flow. Hence, the small scale motion should depend only on the kinematic viscosity and the rate at which the energy is supplied from the large scale motion. Dimensional analysis then gives for the small scale length (Tennekes and Lumley, 1982)

$$
\eta=\left(\nu^{3} / \epsilon_{u}\right)^{1 / 4}
$$




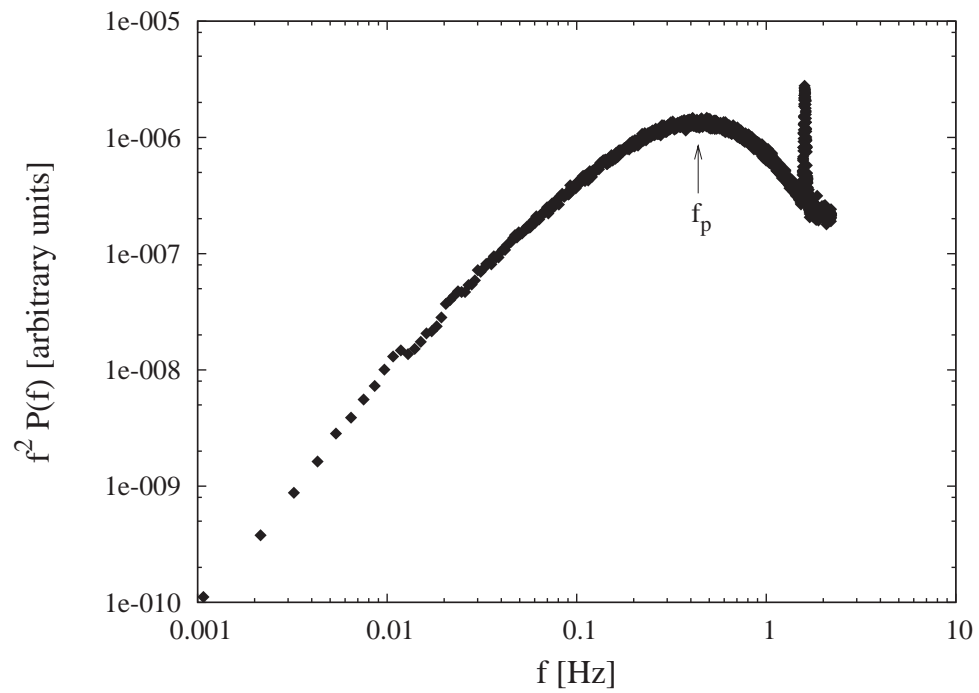

Figure 5.3: Temperature dissipation spectrum at position $20 \mathrm{~mm}$ from the plate. The narrow peak at high frequency end is aliased $50 \mathrm{~Hz}$ noise from the electrical network.

where $\nu$ is the kinematic viscosity and $\epsilon_{u}$ is the rate of kinetic energy supply. It is now reasonable to assume that the rate of energy supply from larger to smaller scales equals the rate of dissipation. The rate of the energy supply is proportional to the reciprocal of the time scale of the larger eddies, $u / L$, where $u$ is the velocity scale of the large eddies. The kinetic energy per unit mass in the large scale turbulence is proportional to $u^{2}$. This gives for the dissipation rate $\epsilon_{u}$ :

$$
\epsilon_{u} \sim u^{3} / L
$$

Thus the dissipation rate can be estimated from large scale dynamics and the Kolmogorov scale can be determined as (Tennekes and Lumley, 1982)

$$
\eta \sim L\left(\frac{u L}{\nu}\right)^{-3 / 4}=L \operatorname{Re}^{-3 / 4}
$$

For the Rayleigh number $\mathrm{Ra} \approx 10^{9}$ typically used in the experiments the Reynolds number $\mathrm{Re} \approx 1400$ which gives Kolmogorov length of the order of magnitude $1 \mathrm{~mm}$.

Analogous to the Kolmogorov scale thermal fluctuations are smoothed out on the scale determined by the thermal diffusivity $\kappa$. This so called "inner thermal scale" can be estimated as (Grossmann and Lohse, 2004)

$$
\eta_{\theta}=\kappa^{3 / 4} \epsilon_{u}^{-1 / 4}=\operatorname{Pr}^{-3 / 4} \eta
$$

which for $\operatorname{Pr}=7$ and $\eta=1 \mathrm{~mm}$ gives $\eta_{\theta} \approx 1 / 4 \mathrm{~mm}$. 
The thermal diffusion time scale is the time needed for heat to diffuse over the length $L$. For $\kappa=0.144 \mathrm{~mm}^{2} \mathrm{~s}^{-1}$ (water at $20^{\circ} \mathrm{C}$ ) this scale is $L^{2} / \kappa=2.8 \times 10^{5} \mathrm{~s}$.

For well developed thermal convection, characterized with high Rayleigh numbers, the largest and the smallest scales are clearly divided by the range of scales. This range can again be divided into the range of scales where buoyancy is the important driving force and the range of smaller scales where inertial force is dominating. The length dividing these two regions is called "Bolgiano length". This length can be estimated as (Skrbek et al., 2002)

$$
l_{B}=\frac{\epsilon_{u}^{5 / 4}}{\epsilon_{\theta}^{3 / 4}(g \alpha)^{3 / 2}} \sim \frac{\mathrm{Nu}^{1 / 2} L}{(\mathrm{RaPr})^{1 / 4}},
$$

where $g$ is the acceleration due to gravity, $\alpha$ is the thermal expansion coefficient, $\epsilon_{\theta}$ is the dissipation rate of temperature variance and $\mathrm{Nu}$ is the Nusselt number (ratio of total heat flux to the molecular heat flux). The first expression in (5.5) yields local values of the Bolgiano length if the local dissipation rates are used. The second expression is obtained on dimensional grounds and is an integral quantity characteristic for the entire volume of the apparatus (Skrbek et al., 2002). This characteristic value of $l_{B}$ for the cell at hand is of the order $l_{B} \approx 5 \mathrm{~mm}$.

In the case of temperature fluctuations $T(t)$ the contribution to the total variance from those components in $\hat{T}(f)$ whose frequencies lie between $f$ and $f+\mathrm{d} f$ is given by $|\hat{T}(f)|^{2} \mathrm{~d} f$, where $\hat{T}(f)$ is the Fourier transform of $T(t)$

$$
\hat{T}(f)=\int_{-\infty}^{+\infty} T(t) e^{-2 \pi f t} \mathrm{~d} t .
$$

The power spectrum $P(f)$ represents the dependence of the quantity $|\hat{T}(f)|^{2}$ on the frequency $f$. A specific frequency $f_{p}$ at which the dissipation spectrum $f^{2} P(f)$ (Hinze, 1959) has a peak defines the time scale $f_{p}^{-1}$ at which the dissipation range starts. The temperature dissipation spectrum at position $20 \mathrm{~mm}$ from the plate is shown in Fig. (5.3). The time scale $f_{p}^{-1}$ in that point is $2 \mathrm{~s}$. As going towards the center of the cell the time scale increases and in the center it is about $6 \mathrm{~s}$. Zhou and Xia (2001) found that $f_{p}^{-1}$ in the cell center coincides with Bolgiano time scale (which relates to Bolgiano length $l_{B}$ ) for Rayleigh number range at least from $4.1 \times 10^{8}$ to $1.85 \times 10^{10}$ and for Prandtl number $\operatorname{Pr} \approx 4$ (water).

\subsection{Large scale circulation}

As described in Section 2.4 the coherent large scale flow carries the plumes discharged from a boundary layer to the opposite plate, where they make 


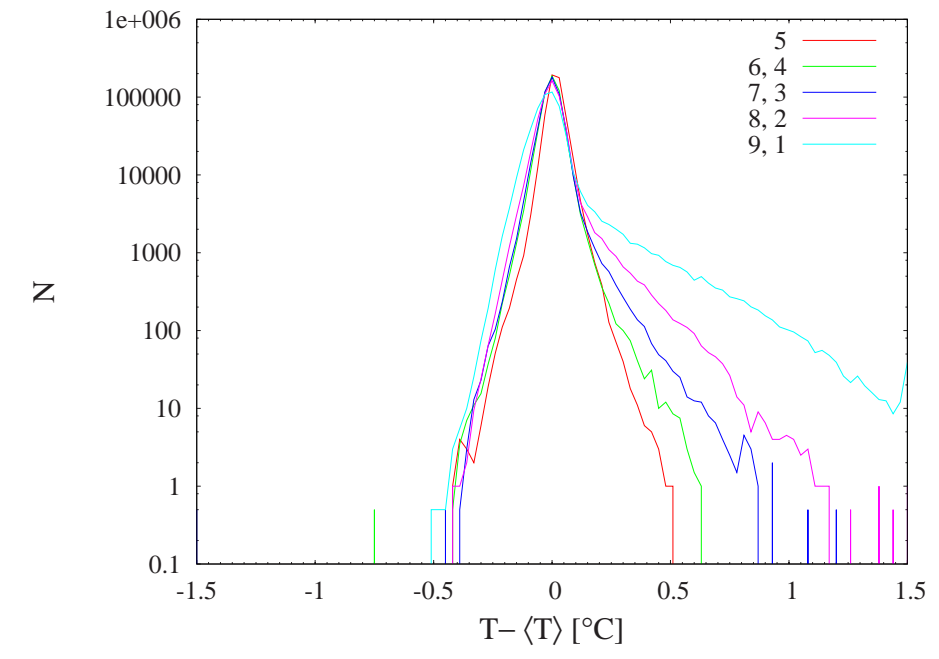

Figure 5.4: Histograms of the temperature fluctuations (averaged over symmetrical positions) from the local mean. Positions are labeled as described in Fig. (5.2).

a perturbation which causes new plumes to be discharged and so on. But the plumes are not only passive objects carried by the mean flow - they "feed" the large scale mean flow through the impulse they exert on the flow as they are ejected from the boundary layers. Thus the "plumes ejection large scale flow" is a self sustaining process. Recently an extensive experimental study of the origin of the large scale flow was performed by means of shadowgraph visualization and PIV measurements (Xi et al., 2004). In that study the answer is given whether the plumes initiate the large scale flow or is it the other way round. In short, the finding is that the plumes appear at the beginning of the process, and that they raise (or fall) vertically without a horizontal velocity component. The vertically moving plumes produce vortices surrounding them and these vortices generate the initial horizontal motion of the flow field. Two types of interactions were identified: $(i)$ direct plume-vortex interaction and (ii) plume-plume interaction via vortices. These interactions as well as the interaction and merging of the vortices lead to grouping or merging of plumes, which in turn generate vortices of larger scale. Finally, a coherent circulatory motion is formed spanning the whole cell.

The large scale circulation has a two dimensional structure and occupies only a limited fraction of the total volume - the "intermediate mixing zone". Its thickness will be estimated in Section 5.3.2. This region is dominated by the plumes. Figure (5.4) shows the histograms of the temperature fluctuations from the local mean. Clearly, the histogram of the temperature fluctuations near the plate is highly skewed due to the large number of plumes in that region, unlike the histogram of the fluctuations in the center of the cell. 

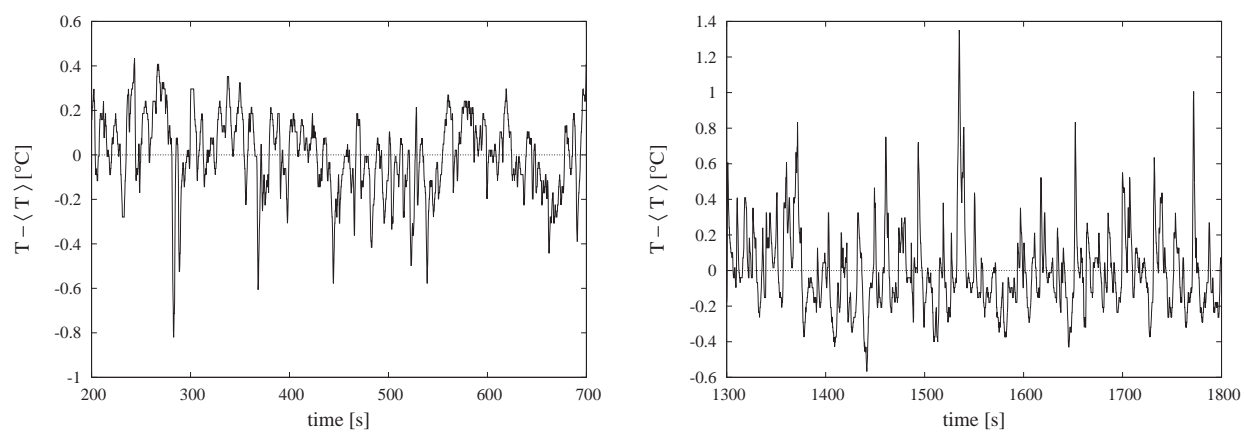

Figure 5.5: Arbitrary chosen sections of the temperature time series $20 \mathrm{~cm}$ from the upper plate (left) and $20 \mathrm{~cm}$ from the lower plate.

\subsubsection{Coherency}

Ejections of plumes from a boundary layer can be easily detected by temperature measurements in the plane of the large scale flow. Figure (5.5) shows temperature time series (with the local mean subtracted) near the top (left picture) and the bottom plate. Obviously there are occasional excursions of hot fluid near the bottom plate and cold fluid near the upper plate and these excursions originate from the plumes. A typical power spectrum of the temperature oscillations is presented in Fig. (5.6).

The peak at $f_{0}=0.011 \mathrm{~Hz}$ in the power spectrum (corresponding to the period of $\approx 90 \mathrm{~s}$ ) can be connected to the velocity of the large scale flow. The key phenomenon responsible for the oscillation is the clustering of plumes through the hydrodynamical interaction as described above. When such a cluster hits the opposite boundary layer a large perturbation causes a large temperature oscillation. Furthermore, a large number of new plumes is produced which makes another cluster and the process repeats. The frequency $f_{0}$ of the low frequency peak in the power spectrum (Fig. (5.6)) is thus the frequency at which the cluster of plumes hits the thermistor. The frequency $f_{0}$ is dependent on the Rayleigh number and it first appears at the transition from soft to hard turbulence, at $\mathrm{Ra}_{c} \approx 5 \times 10^{7}$ (Qiu and Tong, 2001).

It is now of interest to determine the phase between the oscillation on the thermistor $20 \mathrm{~mm}$ beneath the upper plate and the one $20 \mathrm{~mm}$ above the lower plate. The phase $\pi$ would mean that strong oscillations occur alternately at the plates, i.e. that at any given time only one group of plumes exists in the container. A phase 0 would mean that the oscillations occur at the same time, i.e. that at any time there are two "packages" of plumes traveling through the cell. The spectral coherence is the amplitude of the cross spectrum normalized by the original power spectra:

$$
\operatorname{Coh}_{i j}(f)=\frac{\left|\hat{T}_{i}(f) \hat{T}_{j}(f)\right|^{2}}{P_{i}(f) P_{j}(f)}
$$




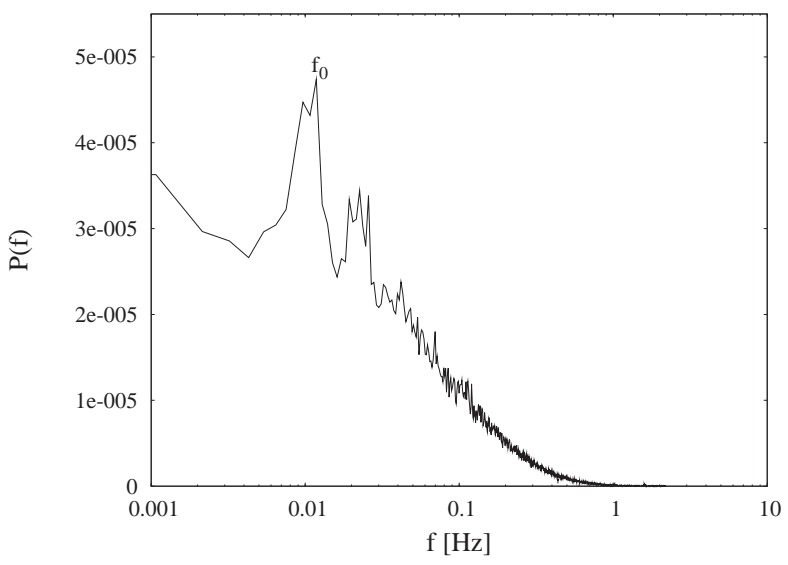

Figure 5.6: Power spectrum of the temperature fluctuation at $40 \mathrm{~mm}$ under the center of the upper plate.
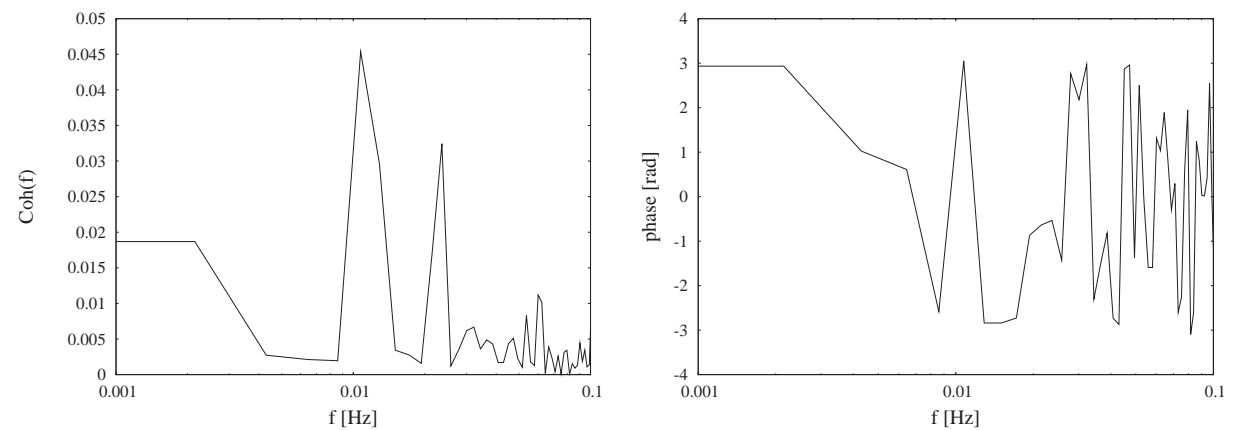

Figure 5.7: Coherence and phase of the thermistors placed $20 \mathrm{~mm}$ beneath the upper plate and the one placed $20 \mathrm{~mm}$ above the lower plate.

Fig. (5.7) shows the coherence and phase of the two thermistors. The phase at the frequency $f_{0}$ is indeed $\approx \pi$ which means that the plume clusters are being emitted alternately from the opposite plates. The coherent oscillation has a frequency $0.011 \mathrm{~Hz}$ as seen from the pronounced peak. Another peak at twice that frequency is also clearly detectable in the coherence as well as in the power spectra. Its origin is explained in Qiu and Tong (2001).

The mean maximal velocity of the large scale flow could not be measured directly in the framework of this thesis, but it can be estimated from other works. For instance, Tilgner et al. (1993) directly measured the velocity at the similar $\mathrm{Ra}$ as in this work, but in a somewhat smaller cell $(L=180 \mathrm{~mm})$ and got $U \approx 6 \mathrm{~mm} \mathrm{~s}^{-1}$. The velocity can also be estimated using the scaling of the Péclet number $(\mathrm{Pe}=U L / \kappa)$. Qiu and Xia (1998) found $\mathrm{Pe}=0.28 \mathrm{Ra}^{0.51}$. For $\mathrm{Ra}=1.5 \times 10^{9}$ used in this work this gives $U \approx 9.8 \mathrm{~mm} \mathrm{~s}^{-1}$. With this velocity $U / 4 L=0.012 \mathrm{~s}^{-1}$ which is very close to $f_{0}$ which proves that the temporal temperature coherent oscillation is 
connected to the (spatial) large scale circulation.

\subsubsection{Spatial modes}

Instead of looking at the temperature at a single thermistor or pair of thermistors as in the correlation or coherency calculations, it is possible to construct a quantity using the instantaneous temperature on all thermistors:

$$
C_{i j}=\sum_{n} T_{i}\left(t_{n}\right) T_{j}\left(t_{n}\right)=C_{j i}
$$

As $C_{i j}$ is a real symmetric matrix it has real eigenvalues and real and orthogonal eigenvectors. These eigenvectors can thus be used to construct mutually uncorrelated modes:

$$
M_{m}(t)=\sum_{i=1}^{N} a_{i m} T_{i}(t), \quad m=1, \ldots N
$$

where $N$ is the number of thermistors (in the experiments nine thermistors were used) and $\left(a_{1 m}, \ldots a_{N m}\right)$ is the m-th eigenvector of $C_{i j}$. The eigenvalues and eigenvectors are calculated as described in Press et al. (1995), Ch. 11.1.

Valuable information can now be gained by studying the power spectra of the time series of the individual modes $M_{m}(t)$. Figure (5.8) shows the eigenvectors and the power spectra of the corresponding modes.

From these pictures it can be seen that the modes which correspond to the strong temperature oscillations at the two outermost thermistors relative to all others contain most power (graphs denoted by $a$ and $i$ ). The next mode containing the most power is the one corresponding to the oscillation of the thermistors 2 and 8 ( $b$ in Fig. (5.8)) whereas the other modes contain insignificant amount of power. This enables identification of the region of thermistors 1 and 2 as the "intermediate mixing zone", that is the region where the large scale flow and plumes dominate. The thickness of the region is thus $\approx L / 4$. Moreover, there is a clearly identifiable oscillation at $\approx 0.01$ $\mathrm{Hz}$ in this region, whose origin is already explained earlier.

\subsection{Coherent structures on intermediate scales}

In order to detect spatial structures on intermediate scales spatial correlation is employed as a convenient tool. The spatial correlation of temperature at positions $i$ and $j$ separated by $\Delta z$ is defined as:

$$
g_{s}(\Delta z)=\frac{\left\langle T_{i} \cdot T_{j}\right\rangle}{\sqrt{\left\langle T_{i}^{2}\right\rangle} \cdot \sqrt{\left\langle T_{j}^{2}\right\rangle}}
$$

where $T_{i}$ and $T_{j}$ are the temperature fluctuations about the local average at positions $i$ and $j$, respectively and angle braces denote temporal averaging. 
a)
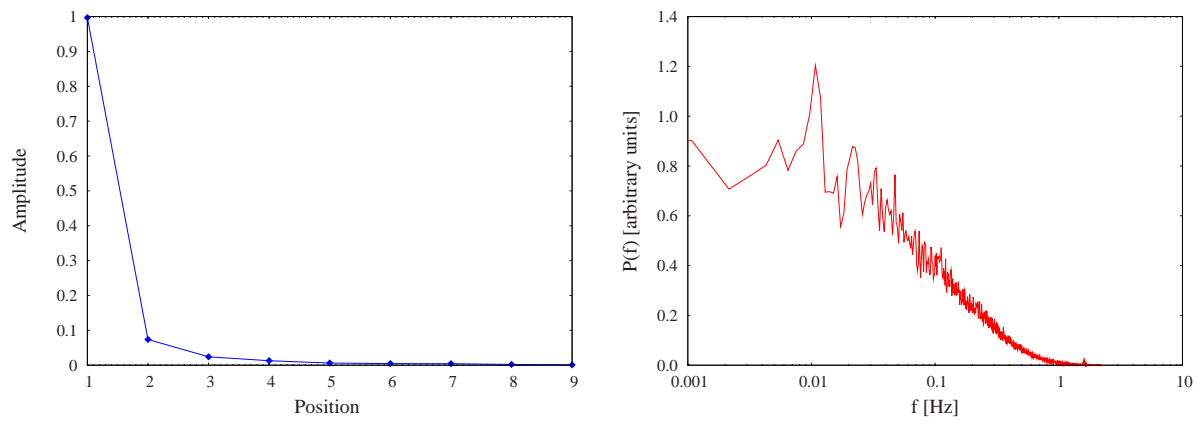

b)
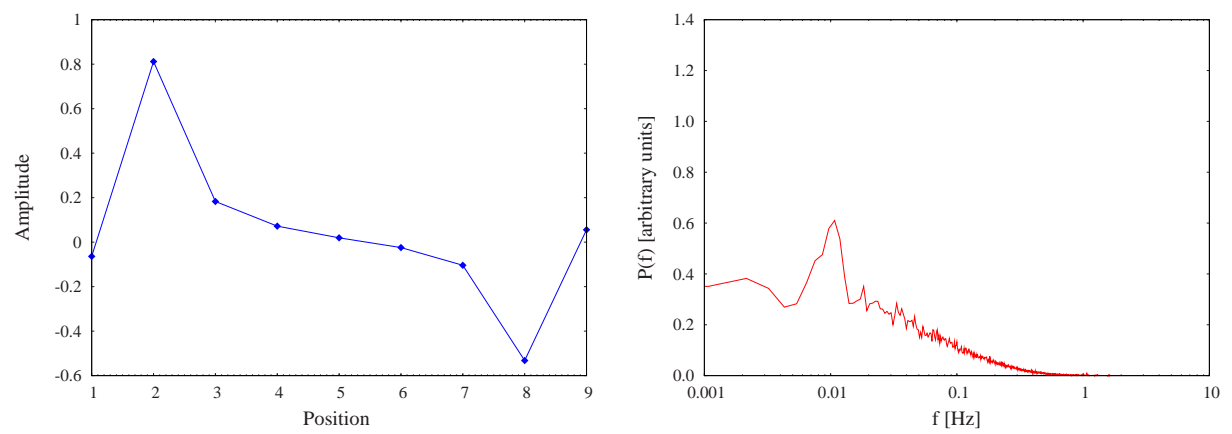

c)
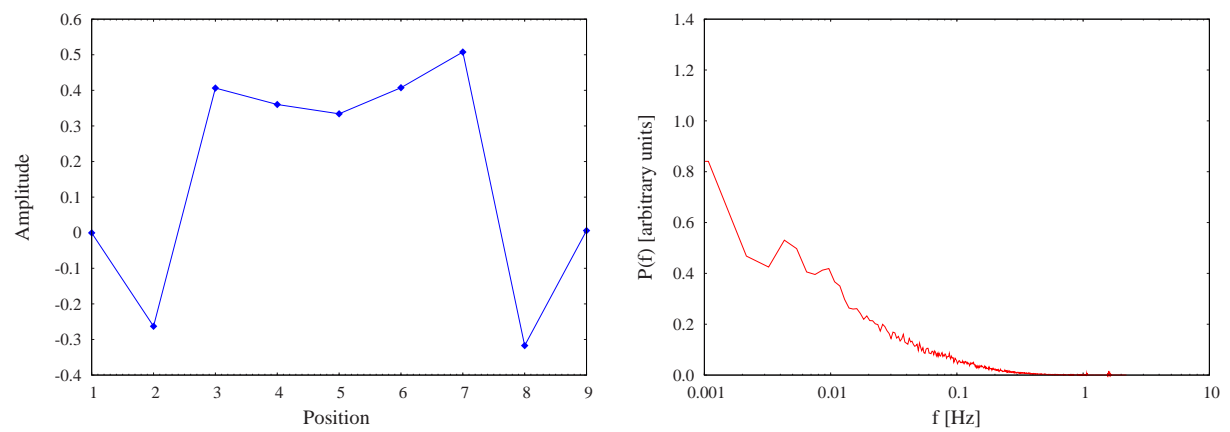

Figure 5.8: Eigenvectors (left) and the power spectra of the corresponding modes. 
d)
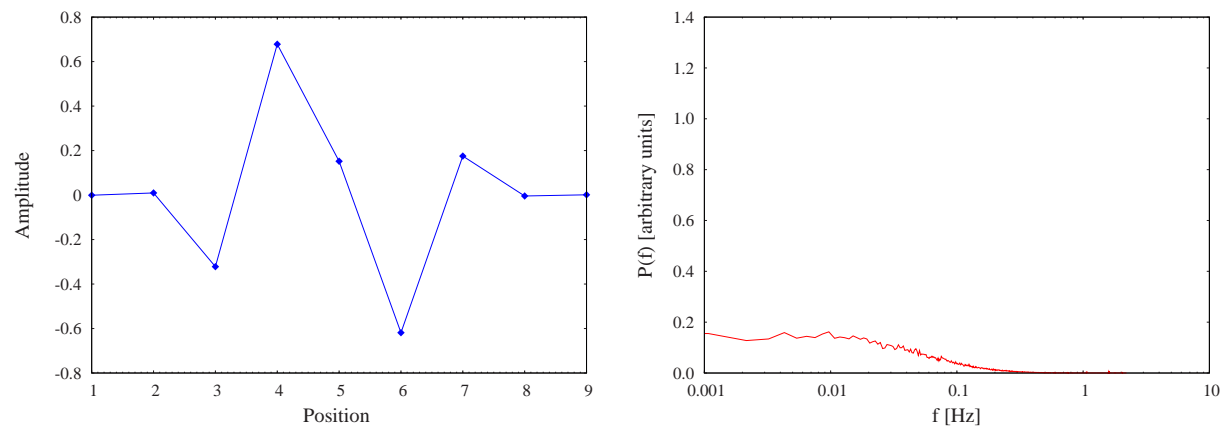

e)
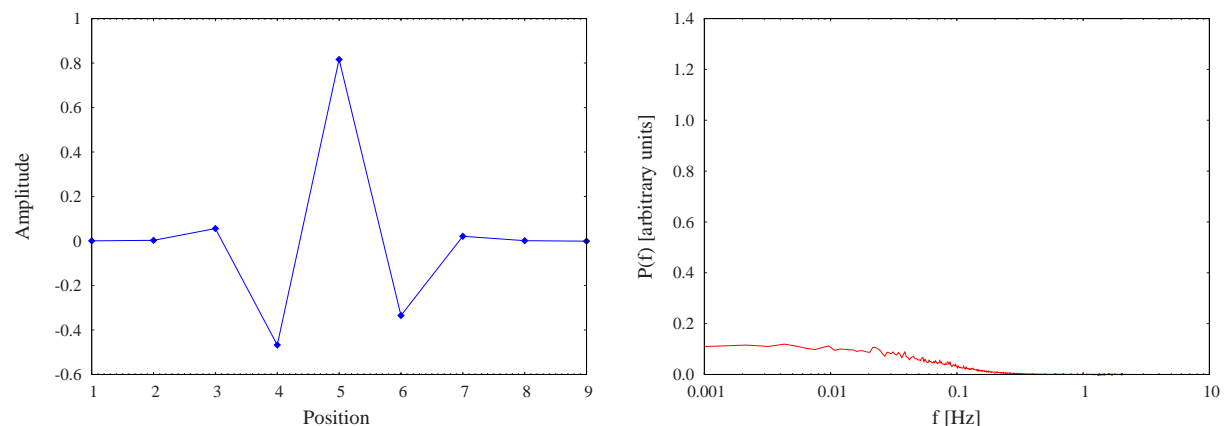

f)
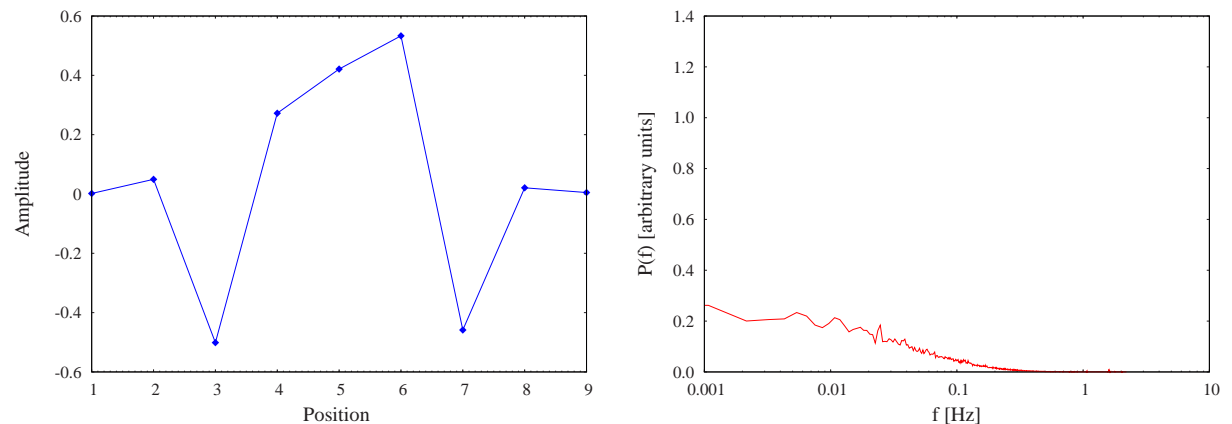

Figure 5.8: (continued) Eigenvectors (left) and the power spectra of the corresponding modes. 
g)
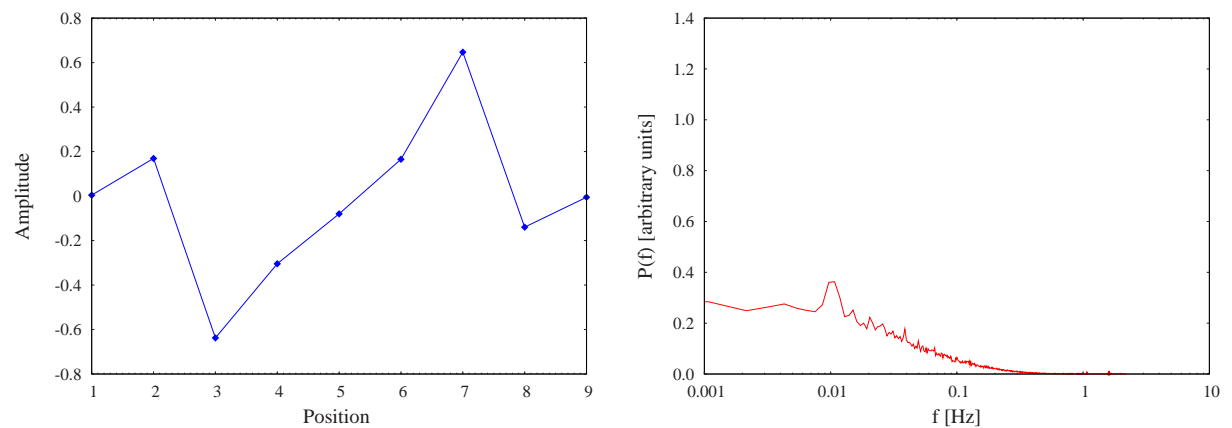

h)
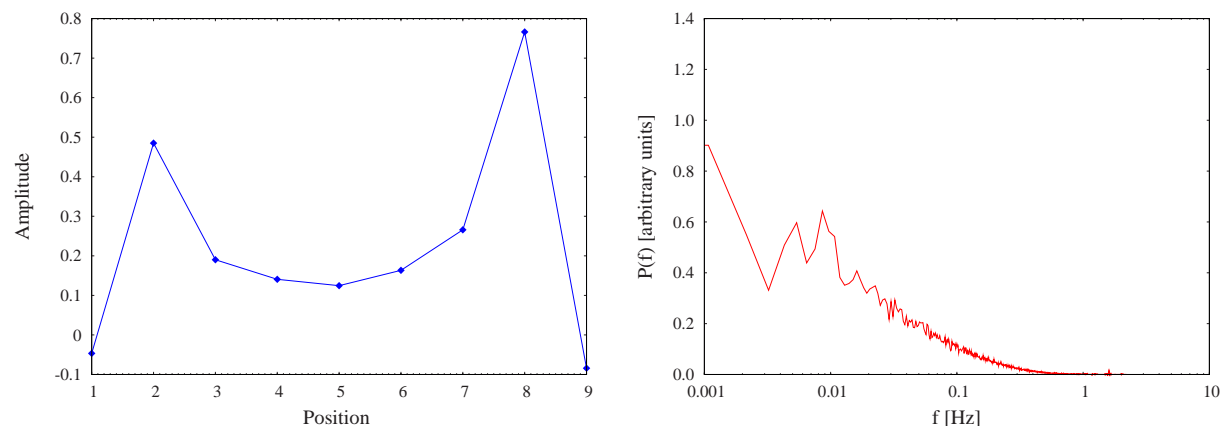

i)
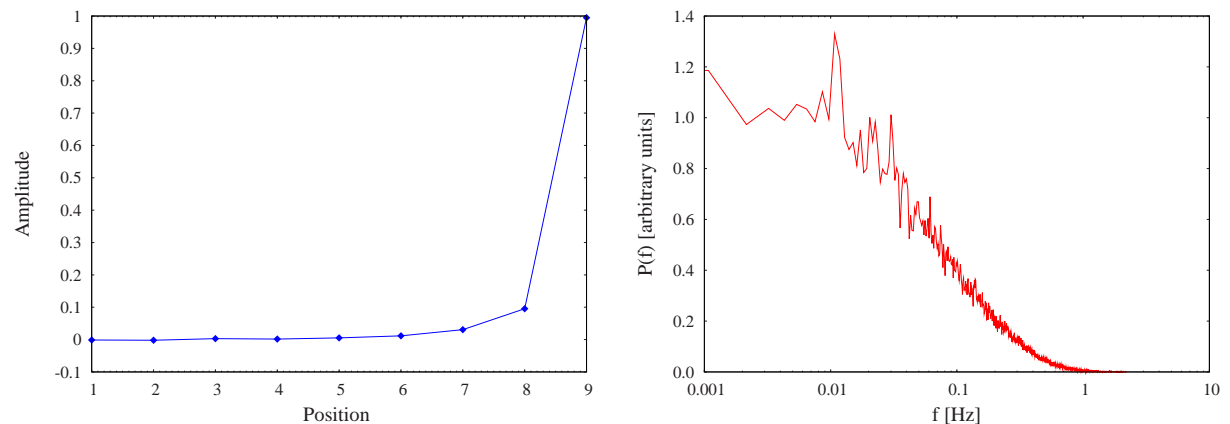

Figure 5.8: (continued) Eigenvectors (left) and the power spectra of the corresponding modes. 
When $\Delta z=0, T_{i}=T_{j}$ and the correlation is equal to 1 by definition. On the other hand, for large $\Delta z$ it is to be expected that the two quantities are uncorrelated, that is $g_{s}(\Delta z) \rightarrow 0$ for large $\Delta z$.

Spatial correlation indicates a distance over which the motion at one point significantly affects that at another (Tritton, 1992), so it may be used to assign a length scale to the turbulent flow. The way the length scale will be defined depends on the general form of the correlation function. For instance, it can be defined as $\int_{0}^{\infty} g_{s} \mathrm{~d} z$, or, if the curve has a negative region, the value of $z$ at which $g_{s}$ is a minimum or at which it first becomes negative, or it can be defined as the distance in which $g_{s}$ falls to $1 / e$ (Tritton, 1992). A scale defined in one of these ways corresponds to the larger structures of the flow. For detection of the small scales (e.g. Kolmogorov scales at which dissipation occurs) other methods are used.

A wish to divide a turbulent flow into an interacting flow of different length scales has its origin in the concept of turbulence as having a cascade of energy from large eddies towards smaller ones, the energy being dissipated by the eddies with the smallest size. Thus a class of eddies is identified by its typical size, or "length scale", different classes of eddies having different roles in the turbulent flow. On the other hand, many researchers challenge such a picture of turbulence. For instance, Warhaft (2000) says, "The experimental evidence shows that the large and small scales are strongly coupled and that the traditional cascade picture, which promotes the notion of universality, is a crude representation". In this Chapter two prominent length scales in the intermediate scale region will be detected and it will be shown using visualization with thermochrystals that the two scales are indeed connected, but it doesn't yet prove neither of the two approaches, especially as no interaction with the dissipation scales can be deduced with the methods used.

In an oversimplified picture an "eddy" is a swirling blob of fluid. A better, but more abstract, definition of an eddy of size $l=2 \pi / k$ (where $k$ is the wave number) is that it is a disturbance containing energy in the vicinity of $k$. It may be now tempting to think of an eddy as a disturbance having a narrow peak in the power spectrum, but that would be a wrong picture. The narrow peak in the power spectrum corresponds to the slowly damped oscillations in the spatial correlation because the correlation and the power spectrum are a Fourier pair (as shown by the equations 5.12). Such oscillations are characteristic of the wavelike disturbances, but not eddies. An eddy is a localized structure and it should loose its identity through interaction with other eddies within one or two of its wavelengths. Therefore, an eddy is a structure with a fairly broad peak in the power spectrum, preventing oscillations in the correlation (Tennekes and Lumley, 1982). 


\subsubsection{Two prominent scales}

In the experiment the spatial correlation is calculated using measurements with the array of nine thermistors arranged as described in Section 5.1 and Fig. (5.2) and one additional movable thermistor. The movable thermistor was positioned on the same vertical axes at different heights between the fixed thermistors. There are two advantages of such a setup. Firstly the correlations can be calculated for many distances and secondly high resolution measurements $(1 \mathrm{~mm})$ can be done in points of interest, which is important if a length scale of the respective size has to be identified.

The interesting result is that the correlations calculated from (5.10) at all positions can be well fitted by the sum of two exponential functions:

$$
g_{t}(z)=a_{0} e^{\left(-z / L_{1}\right)}+\left(1-a_{0}\right) e^{\left(-z / L_{2}\right)},
$$

where $z$ is the separation of the probes and $a_{0}, L_{1}$ and $L_{2}$ are the constants to be found. The experimental correlations and the fit (5.11) are shown in Fig. (5.9). The parameters used in the fit are given in Table 5.1.

In the spirit of the discussion above, constants $L_{1}$ and $L_{2}$ can now be identified as two typical eddy sizes which dominate the flow at intermediate scales. As can be seen from Table 5.1 the two scales differ for one order of magnitude. The smaller scale, $L_{1}$, is of the order of magnitude of the boundary layer, which hints to its origin - the smaller eddies are produced from parts of the detached boundary layer. The bigger eddies have the size of the mixing zone thickness, and are probably produced by shearing in the mixing zone.

Figure (5.10) shows the visualization of the flow in a large part of the upper half of the cell. The thermochromic liquid crystals are blue at the warm end of the temperature range and red-brown at the cold end. The narrow brown stripe just beneath the upper plate is the $\approx 2 \mathrm{~mm}$ thick boundary layer. Two plumes with mushroom like heads can be identified as detached boundary layer. The thickness of these structures is about $5 \mathrm{~mm}$ which equals the size $L_{1}$. The eddies denoted in the picture with "A", "B" and "C" are of the order of magnitude of $L_{2}$. More photos taken randomly

Table 5.1: Parameters used in the fit shown in Fig. (5.9). Distance from the plate is normalized with the height of the cell.

\begin{tabular}{cccc}
\hline Distance from the plate & $a_{0}$ & $L_{1}[\mathrm{~mm}]$ & $L_{2}[\mathrm{~mm}]$ \\
\hline 0.1 & 0.82 & 5.2 & 37.2 \\
0.2 & 0.81 & - & 43.4 \\
0.3 & 0.77 & 6.2 & 39.0 \\
0.4 & 0.65 & - & 30.8 \\
0.5 & 0.46 & 5.4 & 25.8 \\
\hline
\end{tabular}



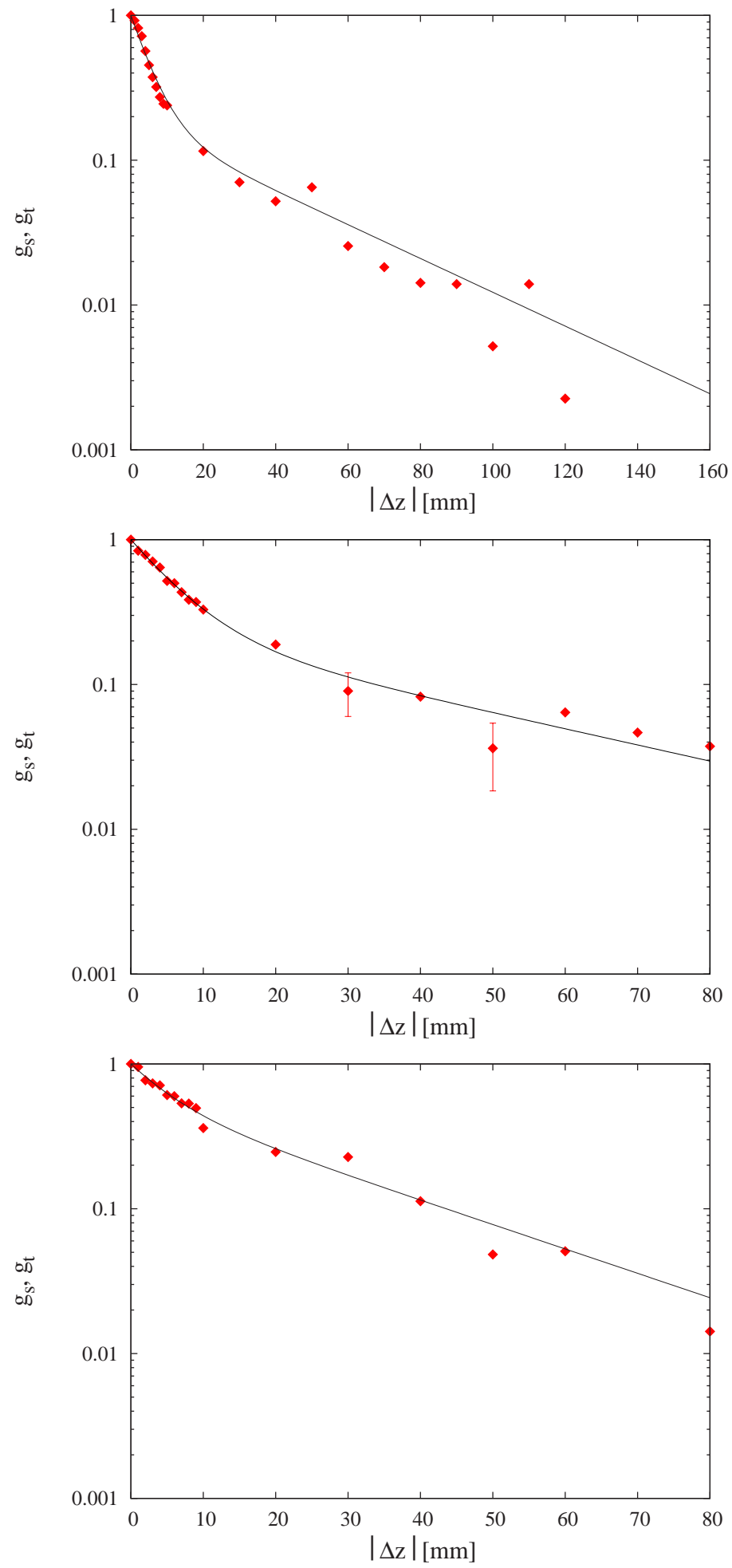

Figure 5.9: Spatial correlations of the temperature at (from top to bottom) 20 $\mathrm{mm}, 60 \mathrm{~mm}$ and $100 \mathrm{~mm}$ from the plate (points) and the fit to the sum of two exponential functions given by (5.11) (solid line) in semi-log scale. 


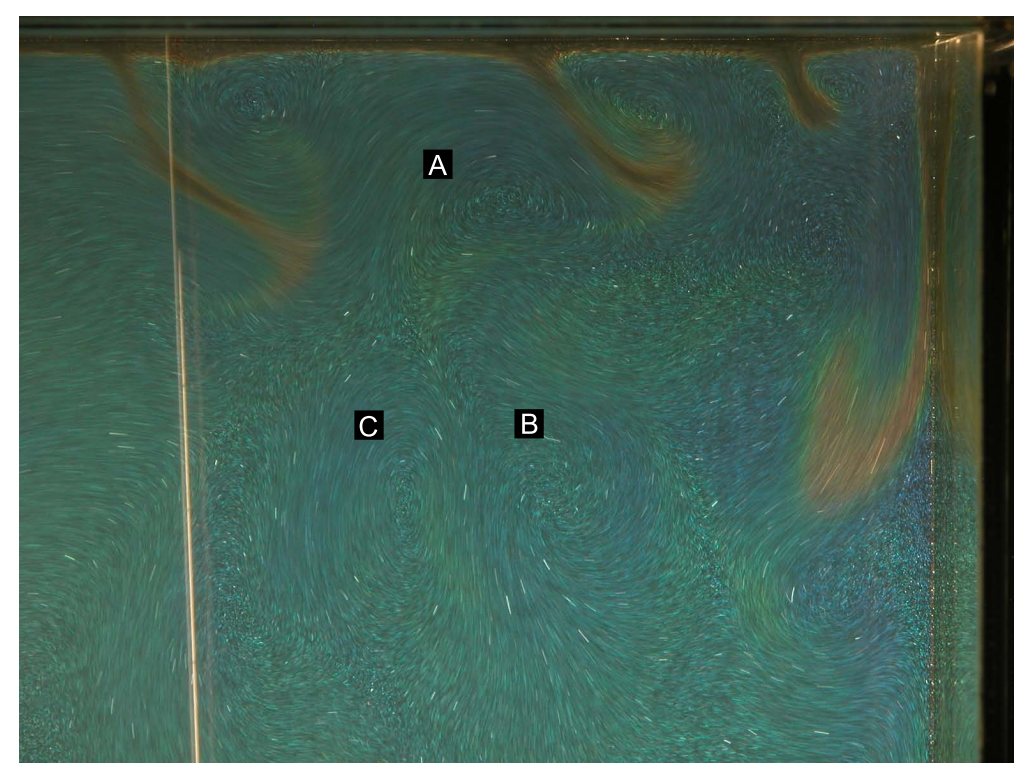

Figure 5.10: Visualization of the flow in the upper half of the cell using thermocrystals. With "A", "B" and "C" are denoted the eddies with size $L_{2}$. For details see the text. The vertical bright line is the holder for thermistors positioned along the central axis of the cell.

in time are presented in Appendix $\mathrm{C}$ to show that the characteristics of the flow in Fig. (5.10) is not a special case, but that these two scales are indeed dominating in the scale region investigated. The visual attractiveness of the photos is certainly another reason to show them in the Appendix.

The dependence of the scales $L_{1}$ and $L_{2}$ on the distance from the plate are shown in Fig. (5.11). The larger eddies have its maximum size 43.4 $\mathrm{mm}$ in the mixing zone and become somewhat smaller as going towards the center of the cell where they are $25.8 \mathrm{~mm}$ in size. On the other hand the eddies which originate from the boundary layer have approximately constant value throughout the cell.

According to the Wiener-Khinchin Theorem the Fourier transform of the spatial correlation is the spatial power spectrum:

$$
\begin{gathered}
g_{t}(z)=\int_{-\infty}^{\infty} P(k) e^{2 \pi i k z} \mathrm{~d} k \\
P(k)=\int_{-\infty}^{\infty} g_{t}(z) e^{-2 \pi i k z} \mathrm{~d} z .
\end{gathered}
$$

If $g_{t}(z)$ is given by (5.11) the corresponding power spectrum is:

$$
P(k)=2\left(\frac{a_{0} L_{1}}{1+4 \pi^{2} k^{2} L_{1}^{2}}+\frac{\left(1-a_{0}\right) L_{2}}{1+4 \pi^{2} k^{2} L_{2}^{2}}\right) .
$$

Now the relative contributions of the eddies with sizes $L_{1}$ and $L_{2}$, respectively, to the total power can be calculated. The total power of the eddies 


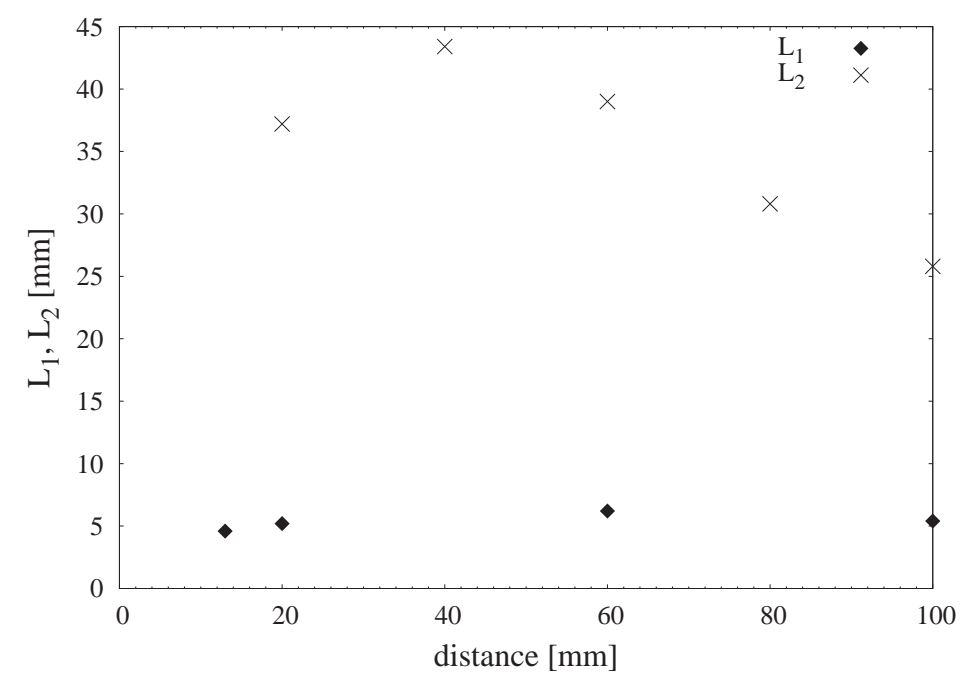

Figure 5.11: Dependence of the size of the eddies on the distance from the plate.

with size $L_{1}$ reads

$$
P_{1}=2 \int_{0}^{\infty} \frac{2 a_{0} L_{1}}{1+4 \pi^{2} k^{2} L_{1}^{2}} \mathrm{~d} k=a_{0} .
$$

Similarly the contribution to the total power from the eddies with size $L_{2}$ is

$$
P_{2}=2 \int_{0}^{\infty} \frac{2\left(1-a_{0}\right) L_{2}}{1+4 \pi^{2} k^{2} L_{2}^{2}} \mathrm{~d} k=1-a_{0} .
$$

The total power $P$ is equal to unity by definition (it is the Fourier transform of the correlation function which equals one at the origin), so that the relative contributions of the small and large eddies to the total power equal the values of the prefactors $a_{0}$ and $\left(1-a_{0}\right)$, respectively.

The dependence of $P_{1} / P$ on the distance from the plate is shown in Fig. (5.12). $P_{1} / P$ decreases from 0.82 near the plate to 0.46 in the bulk center, that is, the small scale eddies dominate near the plate whereas the large scale ones are equally developed in the center of the cell. The fact that the small eddies contribute more to the total power near the plate is not surprising as they originate from the detached boundary layer. It is though at first sight somewhat surprising that they contribute about $50 \%$ to the total power even in the center of the cell. It must be kept in mind though that this is the relative contribution to the total power and the total power is quite small there compared to the mixing zone, so that the total number of small edddies reaching the core is indeed small as well as the number of big eddies. 


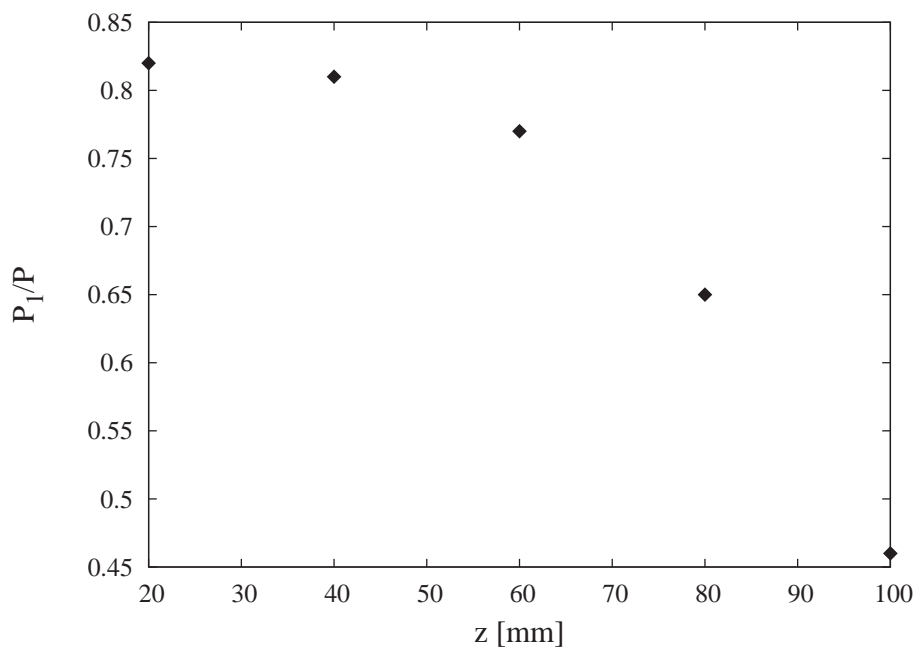

Figure 5.12: Relative contribution of the smaller eddies to the total power as dependent on the distance from the plate.

\subsubsection{Temperature as passive scalar}

Because of buoyancy, temperature is in Rayleigh-Bénard convection generally regarded as an active scalar rather then a passive one. However, Zhou and Xia (2001) found a characteristic passive scalar scaling of the secondorder temperature structure function in a certain region of the container in water at Rayleigh numbers between $4.1 \times 10^{8}$ and $1.85 \times 10^{10}$ and for Prandtl number $\operatorname{Pr} \approx 4$. Also in mercury temperature was found to show a passive character (Segawa et al., 1997). On the other hand, Belmonte and Libchaber (1995) found that temperature is not a passive scalar in gas. As Zhou and Xia (2001) state, these are not necessarily contradicting results, as the Prandtl number varies significantly in the experiments, and a difference in Prandtl number implies a difference in Reynolds number for the same Rayleigh number.

A characteristic of a passive scalar in a turbulent velocity field is the formation of narrow fronts. Large scale velocity structures (eddies) form converging and diverging separatrix and saddle points. If a passive scalar field (temperature) exists in the flow, a temperature front will occur at the diverging separatrix. The front separates the cool and warm fluids entrained by the two counter flowing structures (Warhaft, 2000). A result of the formation of such structures is the typical "cliff-ramp" structure (strong gradient - weak gradient) in the temperature time series (e.g. Shraiman and Siggia (2000), Holzer and Siggia (1994), Warhaft (2000)) because of a strong gradient in a front and a weak gradient in the eddy which follows. The cliff-ramp structures are large scale structures (order of the larger eddies), but the front itself is sharp and thus manifested at small scales. This introduces direct 
interaction of large and small scales and contradicts the simple cascading idea of turbulence. It is interesting to see if such structures exist in the flow at hand - if yes, they could provide an alternative explanation for the source of small scale structures (of size $L_{1}$ ) detected in the flow, especially in the core region where visualization proves that the plumes are quite rare.

Firstly, the dissipative scale of the passive scalar will be determined, so that it can be compared with $L_{1}$. The characteristic magnitude of the velocity gradient is (Shraiman and Siggia, 2000)

$$
\gamma=\frac{v}{L_{2}},
$$

where $v$ is the rms velocity fluctuation at a point and $L_{2}$ is the large scale in which the scalar is stretched and rolled up. Because of incompressibility, the scalar acquires structures on progressively smaller scales $l(t)$. Molecular diffusion becomes dominating on the scale where the diffusion rate becomes comparable to the rate of strain, $\kappa / \eta_{p}^{2} \approx \gamma$. This defines the dissipation scale for the passive scalar:

$$
\eta_{p} \approx\left(\frac{\kappa L_{2}}{v}\right)^{1 / 2}=L_{2} \mathrm{Pe}^{-1 / 2} .
$$

Using the rms velocity $v$ from Tilgner et al. (1993) and $L_{2}$ determined above (Table 5.1) the dissipation scale for temperature as passive scalar $\eta_{p}$ is about $2 \mathrm{~mm}$ throughout the cell. This is about a factor of two smaller then $L_{1}$, which makes the assumption reasonable that structures with size $L_{1}$ are (at least partially) due to the strong gradient temperature fronts. As shown in Fig. (5.13), the cliff-ramp structures can indeed be found in the temperature time series together with symmetric peaks originating from plumes.

In Fig. (5.14) another cliff-ramp structure is shown. The beginning and the end of the structure are marked with arrows. The horizontal dashed lines denote the rms temperature at the point. The size of the whole structure (between the two arrows) is $14.8 \mathrm{~s}$ and the front - from the beginning to the peak of the structure - lasts for $1.4 \mathrm{~s}$. That is, the front is an order of magnitude smaller then the whole structure.

Although the structures such as those presented in Figures (5.13) and (5.14) are shown to exist in the flow, they are relatively rare in comparison with the symmetric peaks which are signature of plumes. Also, the amplitude of the cliff-ramp structures are generally smaller then the amplitudes of the plumes, this difference being larger in the intermediate mixing zone and smaller in the center of the container. Thus, the conclusion can be made that the temperature in Rayleigh-Bénard convection (at least at Rayleigh and Prandtl numbers in this work) partly behaves as a passive scalar and contributes to the formation of the structures on the smaller scale $L_{1}$ through formation of fronts, but this contribution is smaller then the contribution of the plumes. A more precise quantitative investigation of the significance of 


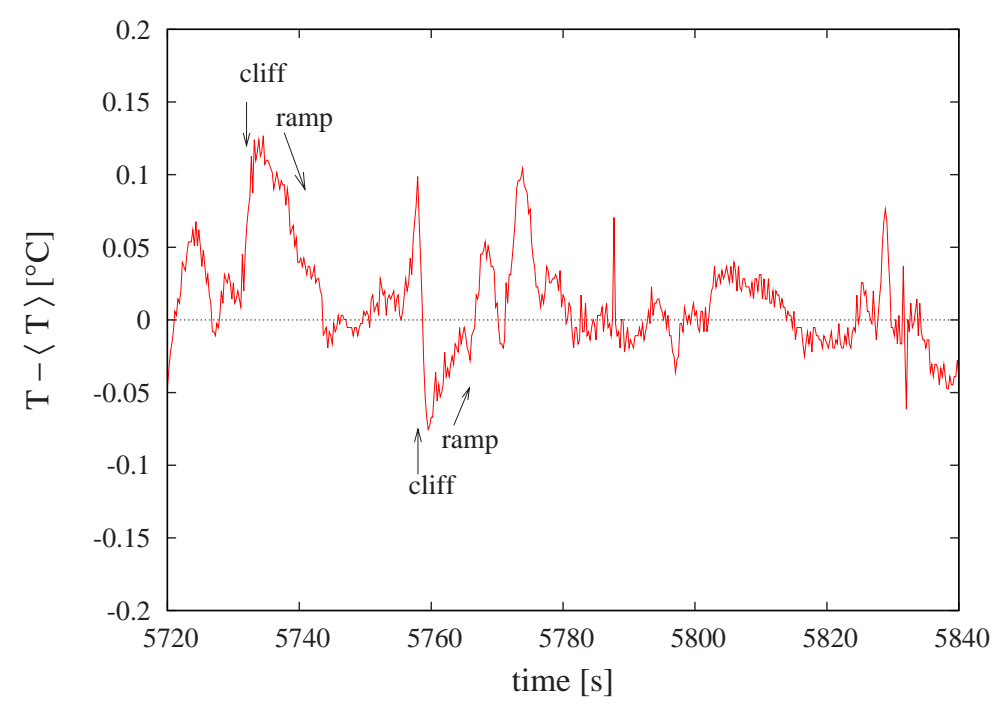

Figure 5.13: Cliff-ramp structures in the temperature time series measured in the cell center.

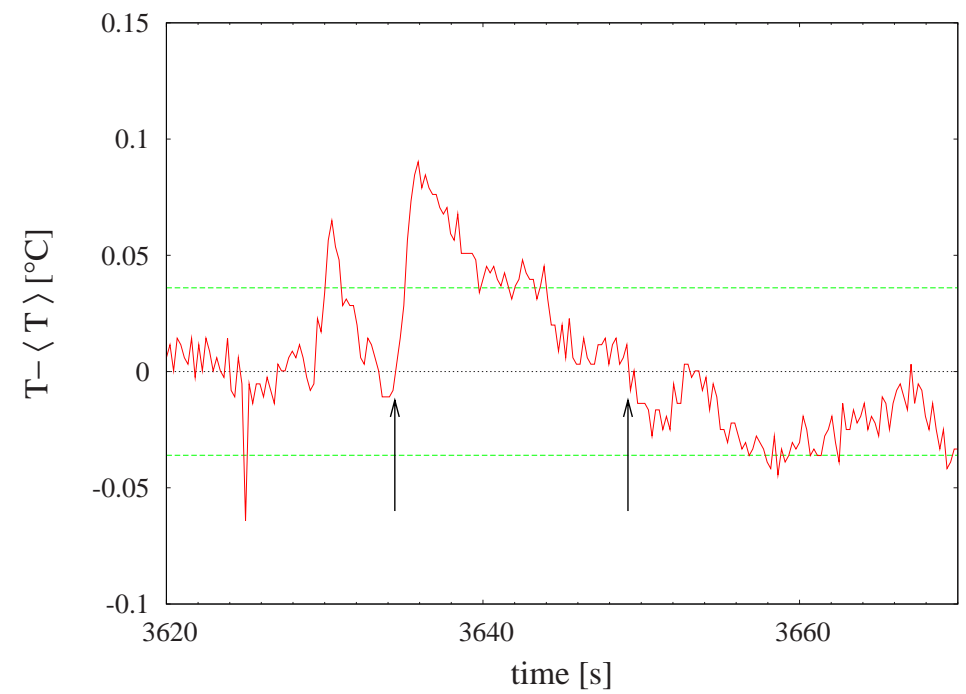

Figure 5.14: Typical size of a cliff-ramp structure in a part of the temperature time series. Horizontal dashed lines denote rms temperature at the point. 
the relative contributions could be made by using the thermometry with the thermocrystals, because the particles can be used both to visualize the large eddies and to measure the temperature gradients at their edges (as seen in the photos in Appendix C).

\subsubsection{Taylor hypothesis}

In experimental work it is quite inconvenient to measure the spatial power spectrum of temperature directly because it would require a dense array of thermo-sensors in the flow. That is the reason that measured spectra are mostly in the frequency domain. On the other hand theoretical work often refers to spatial spectra. For this reason it is convenient to have a way to relate the two of them. In the case where there is a large mean flow $U$ such that the small fluctuations are swept by it this can be done using the Taylor hypothesis (Tennekes and Lumley, 1982)

$$
k=\frac{f}{U},
$$

where $f$ is the frequency and $k$ is the wave number. In the case where the mean advection velocity vanishes the relation between wave number and frequency is not so obvious any more. Nevertheless it was suggested that the spatial and time spectra are then related by the sweeping relation (L'vov, 1991)

$$
k=\frac{f}{v},
$$

where $v$ is the root mean square velocity.

In the experiment the temperature power spectra are measured in the frequency domain at the points shown in Fig. (5.2) and the spectra in the wave number domain are calculated from (5.13). As the temperature is measured along the vertical axis through the cell center the mean flow at the measurement points is either zero (in the cell center) or horizontal (elsewhere) so the relation (5.19) should be used. The spatial spectrum (5.13) can be transformed to its frequency counterpart and then fitted to the measured spectra at respective places in the cell by choosing the velocity $v$. By inspection of $v$ determined this way and the agreement of the measured and calculated spectra it is possible to test the validity of Taylor hypothesis.

The total power is

$$
P=\int_{-\infty}^{\infty} P(f) \mathrm{d} f=\int_{-\infty}^{\infty} P(k) \mathrm{d} k,
$$

so that

$$
P(f) \mathrm{d} f=P(k) \mathrm{d} k .
$$

From (5.19) is $\mathrm{d} k=1 / v \mathrm{~d} f$, so that

$$
P(f)=\frac{1}{v} P(k) .
$$



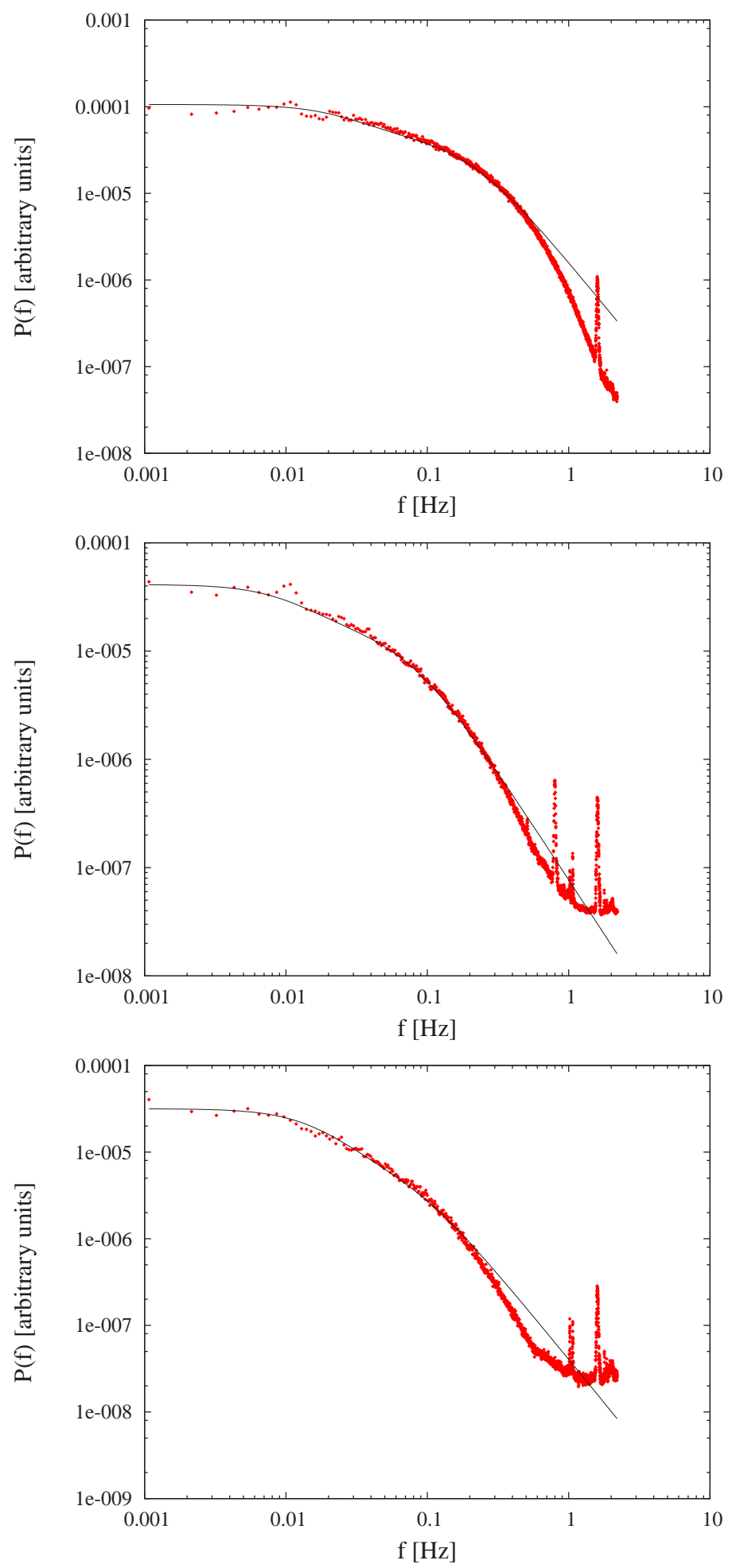

Figure 5.15: Measured power spectra (diamonds) and the power spectra given by (5.23) (solid lines) at positions, top to bottom, $20 \mathrm{~mm}, 60 \mathrm{~mm}$ and $100 \mathrm{~mm}$ from the plate. 


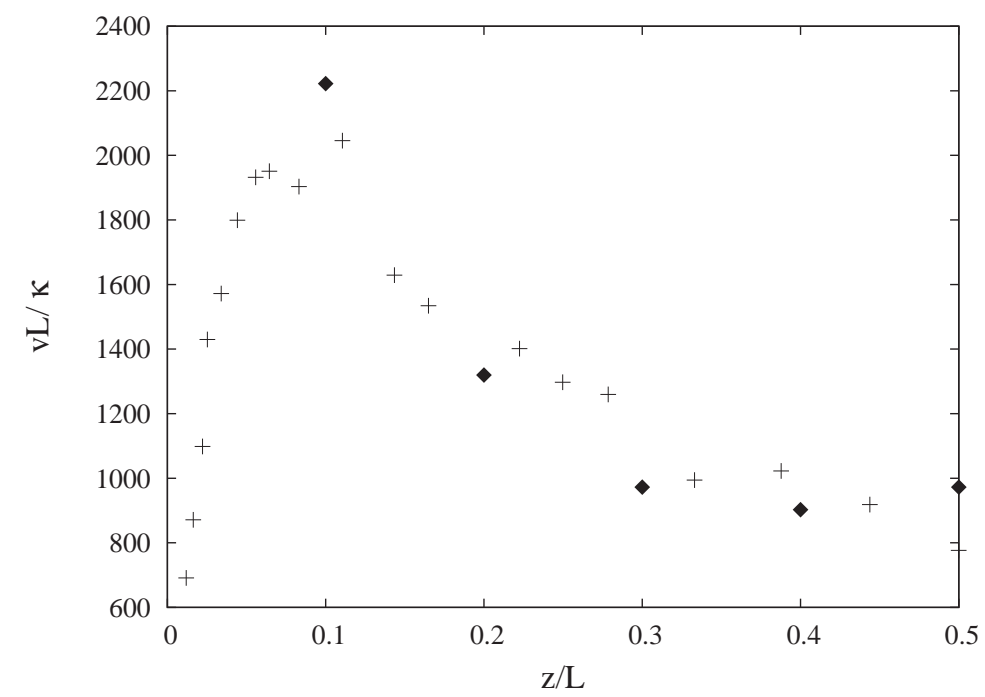

Figure 5.16: Velocity $v$ used to fit the power spectra (5.23) to the measured power spectra scaled down with the factor of 4 (diamonds) and the root mean square of the vertical velocity fluctuations in a similar cell at the same Rayleigh number from Tilgner et al. (1993) (crosses). Velocities are made nondimensional using the height of the cell and the thermal diffusivity, and the distance from the plate is scaled with the height of the cell.

The temporal power spectrum is then

$$
P(f)=2\left(\frac{a_{0} L_{1} / v}{1+4 \pi^{2} f^{2}\left(\frac{L_{1}}{v}\right)^{2}}+\frac{\left(1-a_{0}\right) L_{2} / v}{1+4 \pi^{2} f^{2}\left(\frac{L_{2}}{v}\right)^{2}}\right) .
$$

Figure (5.15) shows the fits at positions $20 \mathrm{~mm}, 60 \mathrm{~mm}$ and in the center of the cell using previously determined $L_{1}, L_{2}$ and $a_{0}$ (Table 5.1). The theoretical spectrum (5.23) can be well fitted to the measured spectrum in the large and inertial scale range (about two and a half decades). It starts to deviate from the measured spectrum at about the frequency $f_{p}$ (Section 5.2 and Fig. (5.3)) where the dissipation range starts. There are two possible reasons for this discrepancy: either the exponential function is not appropriate approximation for the correlation at small scales (scales in the dissipation range) or the Taylor hypothesis is not valid in that range.

It can be shown that the correlation at the origin must have first derivation equal to zero, so that there is a certain range about the origin where exponential function is not appropriate approximation. However, the discrepancy in the power spectrum stretches to the scale as large as Bolgiano length and in that range there are enough measurement points to prove that the exponential function is indeed a good fit for the correlation. Thus, although there is some small scale where the exponential function is not a good approximation it is certainly much smaller then Bolgiano length. From 
that it can be concluded that possibly inappropriate use of the exponential function as approximation for the correlation at a small scale does not cause the discrepancy in the power spectrum.

The assumption that the Taylor hypothesis is too simple way to transform the spectra seems to be more reasonable. In Fig. (5.16) the velocity $v$ (scaled down with the factor of about 3) used in (5.23) for the fits and the root mean square of the vertical velocity fluctuations measured in a similar cell and the same Rayleigh number (Tilgner et al., 1993) are compared. The fact that they have a similar form is a hint that the rms of velocity is indeed important in transforming the spectra between the frequency and wave number domain as supposed by e.g. L'vov (1991) and Ching et al. (2004). On the other hand the difference in magnitude shows that taking rms velocity as only transformation parameter is too simple. That is because not only the fluctuations of the turbulent velocity field plays a role, but probably a complicated interplay of the coherent structures on different scales as well.

\subsection{Overview of scales in the flow}

This Chapter will be closed with an overview of the scales in RayleighBénard convection discussed in previous and in this Chapter. All the scales are measured or calculated for the rectangular container with height 200 $\mathrm{mm}$, aspect ratio one, $\mathrm{Ra} \approx 10^{9}$ and $\operatorname{Pr} \approx 7$.

Table 5.2: Overview of the scales discussed in the thesis.

\begin{tabular}{cc}
\hline Scale & Size in $\mathrm{mm}$ \\
\hline thermal inner scale & 0.25 \\
Kolmogorov scale & 1 \\
thermal boundary layer thickness & 2 \\
thermal front (passive scalar) & 2 \\
smaller intermediate scale $\left(L_{1}\right)$ & 5 \\
Bolgiano length & 5 \\
peak in distribution of streak distances & 10 \\
larger intermediate scale $\left(L_{2}\right)$ & 30 \\
container height & 200 \\
\hline
\end{tabular}




\title{
Chapter 6
}

\section{Influence of the container shape on the flow}

\begin{abstract}
$T^{\text {HE }}$ problem of convective flow can be described using nondimensional 1 equations (Chapter 2) and only three nondimensional parameters Rayleigh and Prandtl numbers explicitly and the aspect ratio through the boundary conditions. The fact that the shape of the container does not enter the flow description could provoke the impression that it does not influence the details of the flow (at least away from the boundaries). In fact, the experimental results in small aspect ratio cells (especially the ones with the aspect ratio one, which are often performed), are always compared without regard to the container's shape. Two canonical shapes mostly used in experiments are a cylinder and a cube. In this chapter experimental results are presented which show that the shape of the container actually plays a role in the properties of the flow, including the coherency properties described in the Chapters 4 and 5. The results of this study are of interest to geophysical community as well as for industrial applications. In geophysics the real flows in nature are mostly laterally unconfined, which in laboratory experiments is hard to achieve. For that reason it is important to know the influence of the walls to the experimental results which are then used to explain real natural phenomena. To the industrial applications it may be of interest that change in the shape of a container can change temperature oscillations or pattern formation properties.
\end{abstract}

\subsection{Experiments}

Two types of experiments were performed: (i) visualization near the boundary layers and (ii) temperature measurements at different positions in the cells.

Visualization was used to collect qualitative information about the direction of the velocity field of the large scale flow. It is performed using the $\mathrm{pH}$ 


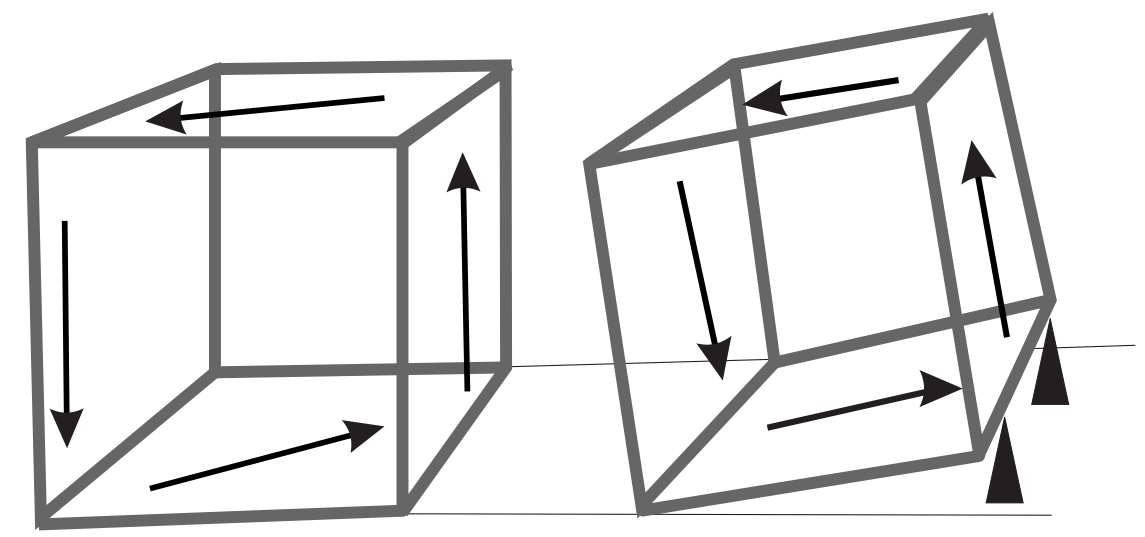

Figure 6.1: Influence of the container tilting on the direction of the flow in a cube. In the leveled cube the flow is across the diagonal on the plates (left) and in the tilted cube the flow is parallel to the sides.

method as described in Section 4.1, the only difference being that a higher voltage was applied between the plates. This has the effect that the dyed layer is thicker then in the experiments with visualization in the boundary layer, so that a part of the large scale flow in the intermediate mixing zone is dyed as well. Photographs of the lower plates were then taken every $2 \mathrm{~s}$ and analyzed. Also they were composed to movies (one for the cylinder and one for the cube) in such a way that the movie shows the velocity field in fast motion, about 10 times faster then in reality. This makes it possible to envisage the variability of the velocity field on slower time scales.

Temperature measurements were performed in different parts of the cell along the vertical axes using the same method and at the same positions as described in Section 5.1.

All experiments were carried out in a cylindrical and a cubical cell. In order to isolate the influence of the shape on the flow, the experiments in the cylinder were done so that the cylindrical tube was inserted in the cube and all other conditions (plates, heating, cooling, insulation) were left the same. The cylindrical tube is made of $3 \mathrm{~mm}$ thick plexyglass and the cubical setup is described in Section 3.1.

Additionally, the experiments were performed in the containers tilted for a small angle (typically $3-4^{\circ}$ ). This has an effect on the large scale flow. In the tilted cylinder the large scale flow can not change its horizontal direction as easily as in the leveled one, it is "locked" in one direction. In the cube the direction of the large scale flow is relatively stable, but by tilting the container its direction can be changed from a flow across the diagonals in the leveled container to a flow parallel to the walls in the tilted container (Fig. (6.1)). Note that the container is supported on one side, not in a corner. 

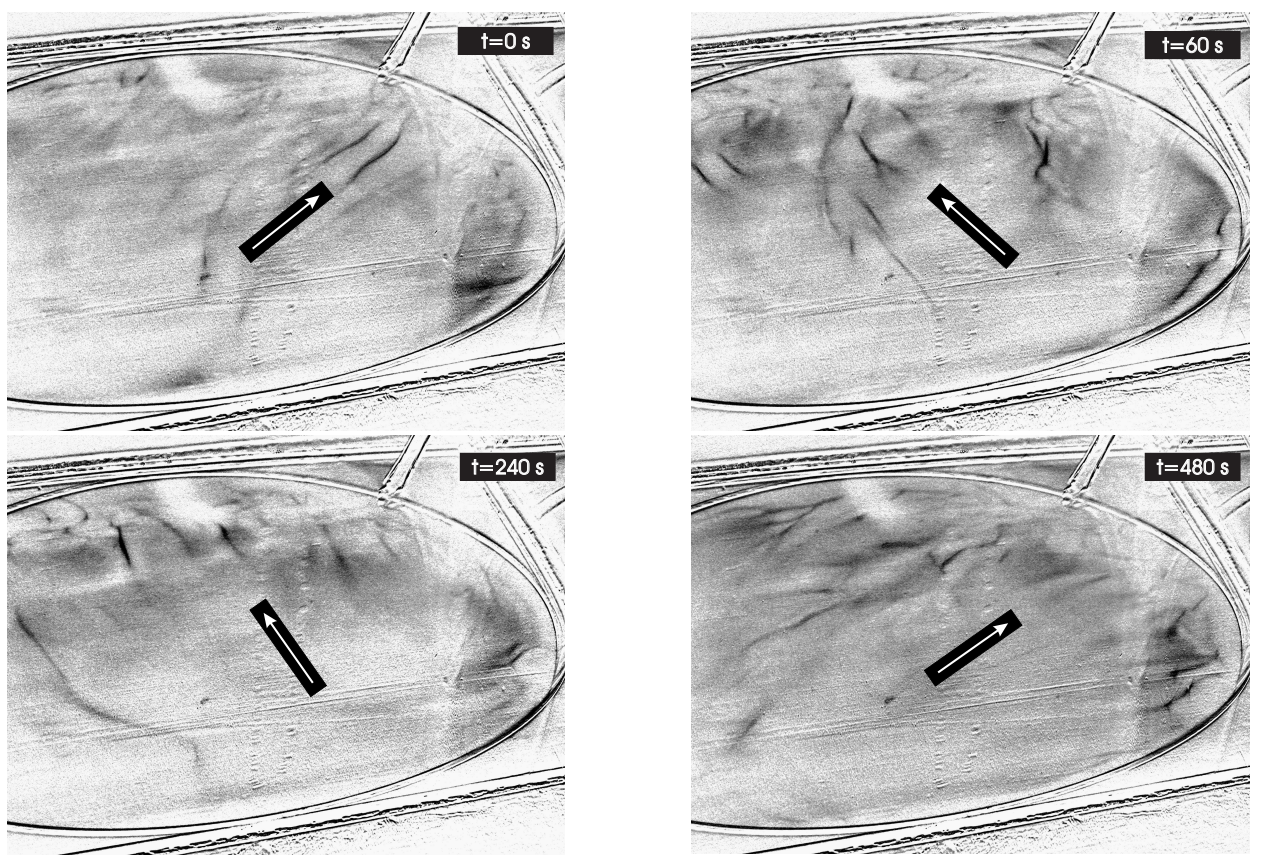

Figure 6.2: Example of the horizontal oscillation of the large scale mean flow in the cylinder. In the course of 8 minutes the flow changed direction for almost $90^{\circ}$.

This method enables investigation of the influence of the large scale flow on the temperature oscillations in the interior of the cell.

\subsection{Velocity field}

The large scale flow velocity above the lower plate in the cube is across the diagonal. Spontaneously the direction can change from one diagonal to the other and this happens roughly at the time scale of one hour. In the cylinder the flow changes its direction much more vividly. The horizontal direction fluctuations occurs on the time scale of roughly one minute and the destruction of the large scale flow as well as the direction reversals are more often then in the cube (actually, the flow reversals were not observed in the cube during the experiments; also, most experimental works about the wind reversals are done in cylindrical cells, e.g. Niemela et al. (2001), K.R.Sreenivasan et al. (2002)). An example is shown in the series of photographs spanning the time of 8 minutes (Fig. (6.2)). In that period the flow oscillates for about $50^{\circ}$ (the direction of the large scale flow is denoted with arrows).

By tilting the cylindrical container for $4^{\circ}$ it can be achieved that the flow becomes more similar to the flow in the cube in the sense that it stays "locked" in one direction for a longer time then in the leveled container. 


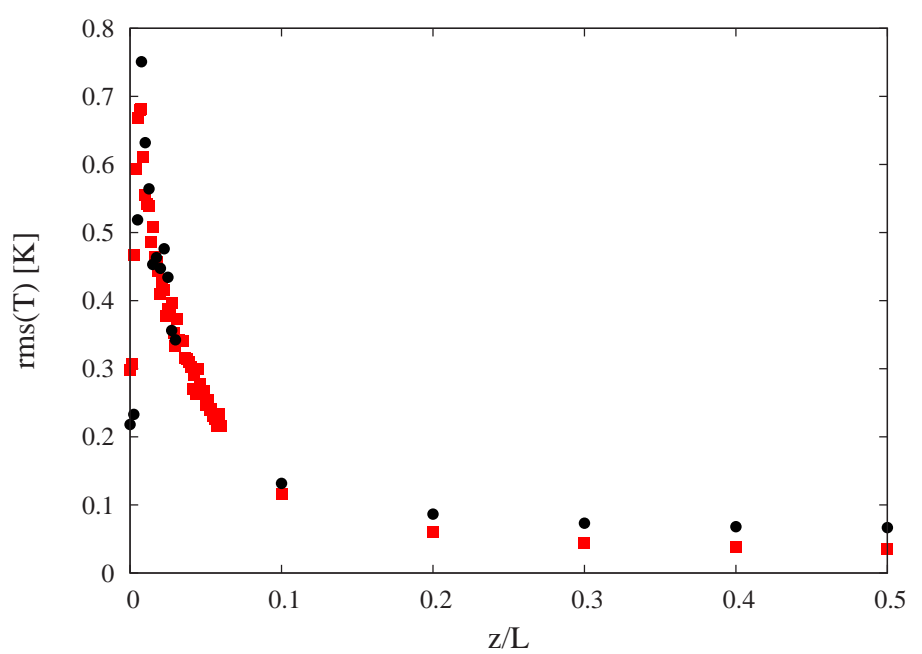

Figure 6.3: Root mean square profiles in the cube (squares) and the cylinder (circles).

Similarly, tilting the cube the flow direction can be affected as sketched in Fig.(6.1). In this way the total path a parcel of fluid travels is smaller then in the case when the flow is across the diagonal and is the same as in the case of a cylindrical container.

By any of these manipulations the magnitude of the velocity was not changed as will be shown later using temperature measurements.

\subsection{Temperature field}

The root mean squares (rms) of the temperature in the leveled cube and cylinder are shown in Fig.(6.3). As the definition for the boundary layer alternative to the one presented in Section 4.3 the position of the maximum rms can be used. Comparison of the rms profiles then shows that the thicknesses of the thermal boundary layers are the same in the cube and the cylinder. No differences can be identified also in the region up to $z / L=0.1$. But at the position $z / L=0.2$ the difference in the rms appears and becomes always larger up to the center of the cell. It is somewhat surprising that the influence of the walls has the strongest effect on the temperature fluctuations at the point with the biggest distance from the walls and no influence on the temperature near the walls.

Figure (6.4) shows the rms profiles in one half of the cells (both leveled and tilted) obtained by averaging the rms' at symmetrical positions labeled 1 to 9 in Fig. (5.2). It can be seen that in the center of the cylindrical cell the root mean square is almost double that in the cubical one if both cells are leveled. Closer to the wall the relative difference becomes smaller, 


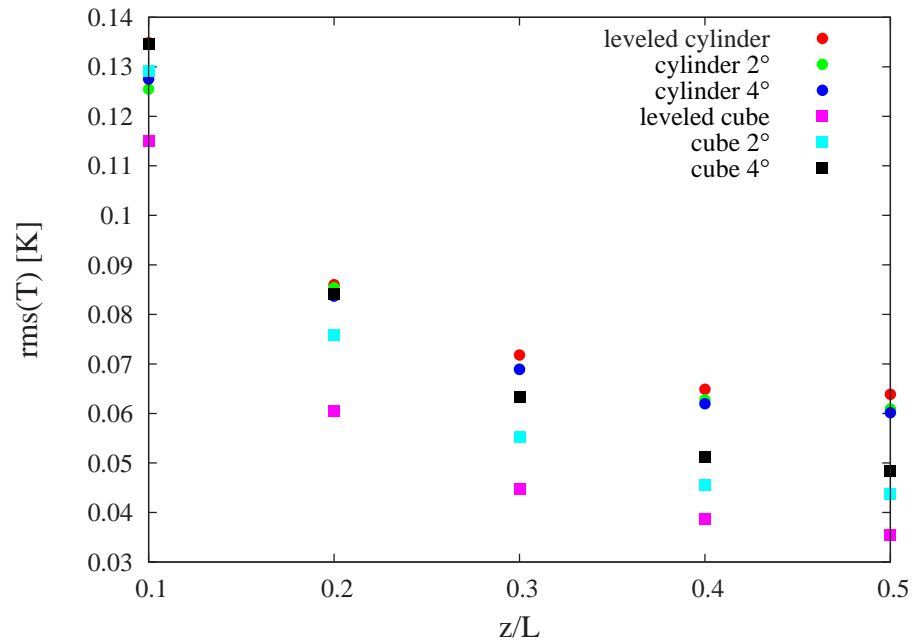

Figure 6.4: Root mean squares in the leveled cubical and cylindrical containers and the containers inclined for $2^{\circ}$ and $4^{\circ}$ at positions 1 to 5 .

but the rms remains always bigger in the cylinder then in the cube in the region shown in the figure. By tilting the containers (that is, by making the large scale flow more similar in the two cells, as explained in the previous section), the rms in the center of the cube increases and the rms in the cylinder decreases, but they do not become the same. On the other hand, they do become of the same magnitude at the positions $z / L=0.1$ and $z / L=0.2$, that is in the region identified in Section 5.3 as "intermediate mixing zone".

In order to find out the reason for such a behavior the histograms of the temperature fluctuation in the central region of the cells are compared as shown in Fig. (6.5). The first assumption was that the fluctuations in the center of the cell are caused by plumes which escape the mixing zone and reach the core and that there is a different amount of them in the different setups. The relative influence of the plumes can be estimated by comparing the tails of the histograms for the centers of the investigated cells. Contrary to the expectation there is no difference in the number of plumes, so that this assumption had to be rejected.

A better explanation gives the investigation of the large scale flow. The temperature time series were recorded at the positions 1 and 9 in the leveled and the inclined containers and the power spectra were calculated. For each container the spectra at the given positions are then averaged. The result is presented in Fig. (6.6). The low frequency peak corresponding to the turnover time as explained in Section 5.3 can be identified in all setups. The peak appears at approximately the same frequency for all containers - $0.0118 \mathrm{~Hz}$ in the leveled cylinder, $0.0128 \mathrm{~Hz}$ in the inclined cylinder, $0.0118 \mathrm{~Hz}$ in the inclined cube and 0.0107 in the leveled cube. In the leveled 


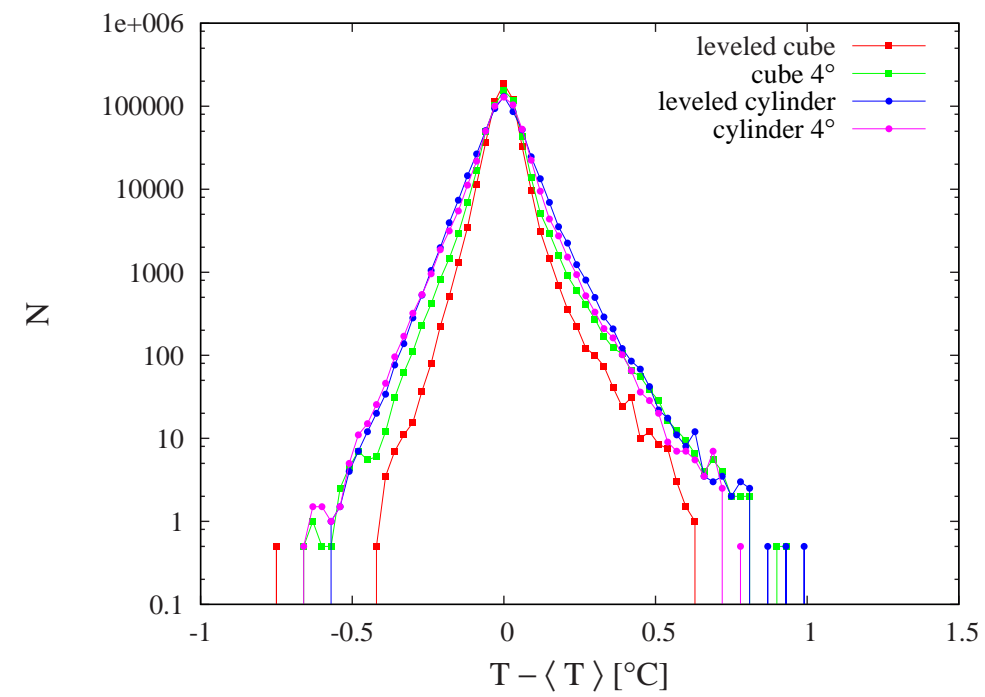

Figure 6.5: Histograms of the temperature oscillations at position 6 in different experimental setups.

cube the turnover frequency is the smallest, but the total path traveled is longer, because the horizontal part of the flow is across the diagonals (that is, the path is for the factor $(2 \sqrt{2}+2) / 4$ longer $)$, so that the velocity can be considered approximately the same all containers. This implies that tilting a container for a small angle does not influence the average velocity of the large scale flow.

The striking difference, on the other hand, is in the width of the peaks. The width of a peak in a power spectrum is a measure of the life time of an oscillation. Thus it can be inferred from Fig. (6.6) that the life time of the large scale rolls are not the same. In many models of convection in a closed box not much attention is payed to the fact that the large scale circulation has a certain finite life time, that is, it appears and disappears - the findings in this thesis proves such models to be oversimplified. Furthermore, the comparison of the power spectra in Fig. (6.6) shows that the life times are shape dependent and thus a good candidate for explanation of the temperature fluctuation dependence on the shape of the container.

According to Grossmann and Lohse (2004) the root mean square of the temperature in the center of the cell scales as

$$
\operatorname{rms}(T) \sim\left(\epsilon_{\text {bulk }} \eta_{\theta}^{2} \kappa^{-1}\right)^{1 / 2}
$$

where $\epsilon_{b u l k}$ is the thermal bulk dissipation rate, $\eta_{\theta}=\kappa^{3 / 4} \epsilon_{u}^{-1 / 4}$ is the inner thermal length scale, $\epsilon_{u}$ is the kinetic bulk dissipation rate and $\kappa$ is the thermal diffusivity. Grossmann and Lohse (2000) estimate the thermal bulk 


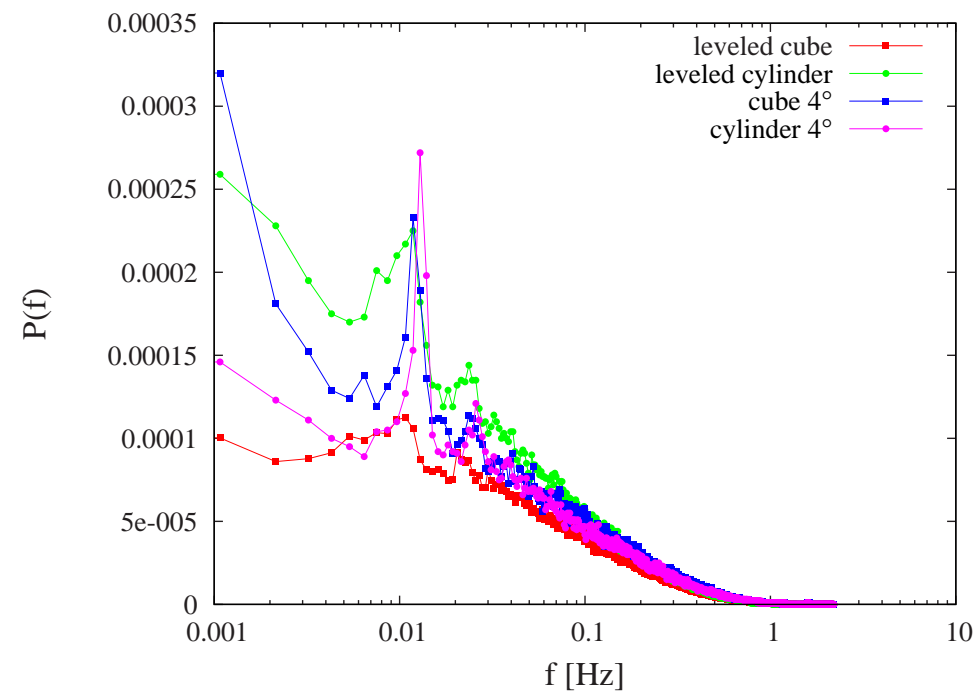

Figure 6.6: Power spectra of the temperature time series in different cells. Each spectrum is average of the spectra at symetrical positions 1 and 9 .

dissipation rate as

$$
\epsilon_{b u l k}^{\prime}=\frac{\lambda_{\theta}}{\lambda_{u}} \frac{U \Delta^{2}}{L}
$$

where $\lambda_{\theta}$ and $\lambda_{u}$ are the thicknesses of the thermal and the velocity boundary layers, respectively, $U$ is the velocity scale (taken to be the average velocity of the large scale flow), $\Delta$ is the temperature scale (difference between the temperatures of the lower and the upper plate) and $L$ is the length scale (height of the container). All scales used in the last two equations are the same in the containers with different shapes and can not reproduce the observed difference. Moreover, Daya and Ecke (2001) found experimentally that the temperature fluctuations in the center of the cells scale differently with Rayleigh number in a cylinder and a cube, which implies that the difference can not be simply in a constant prefactor.

It is now possible to test the assumption that the properties of the large scale roll are responsible for the fluctuations in the center of a cell. These properties result in different power spectra around the turnover frequency. The area under the peak in the power spectrum has dimensions $\left[K^{2} s^{-1}\right]$, that is the dimension of the thermal dissipation rate. It is now possible to take the ratios of the root mean squares in the centers of different containers taking the thermal dissipation rate to be proportional to the area under the peak in the power spectrum, instead of being as given by (6.2). Denoting with subscripts $i$ and $j$ the different experimental setups, the ratios of the 


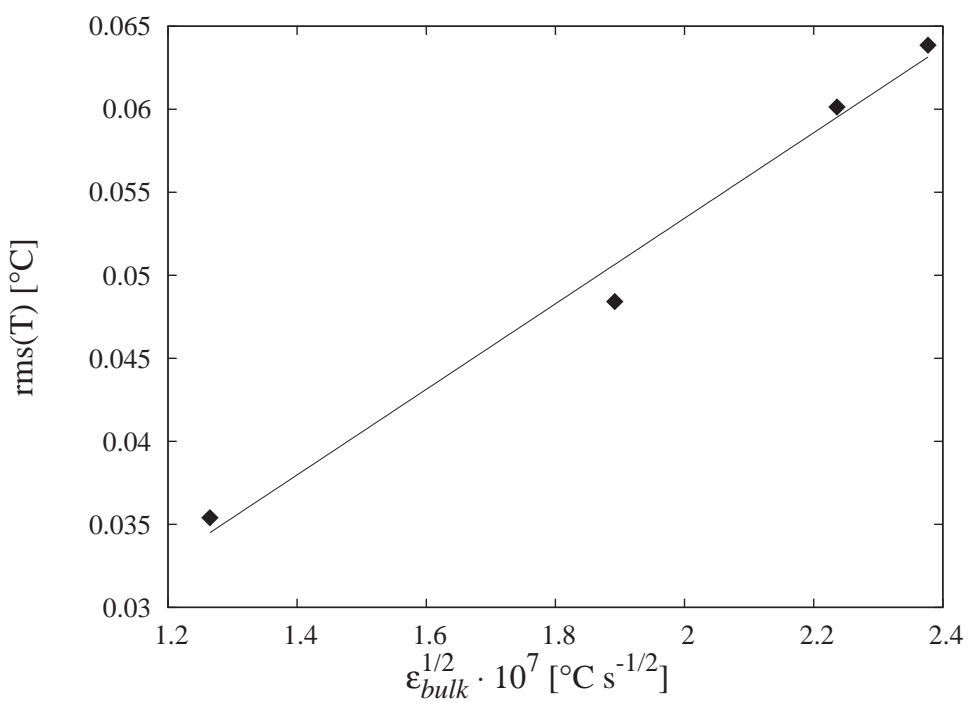

Figure 6.7: Dependence of $\operatorname{rms}(T)$ on $\sqrt{\epsilon_{\text {bulk }}}$. Straight line is a linear fit.

rms' in the center of the cells calculated from

$$
\frac{\operatorname{rms}_{i}(T)}{\operatorname{rms}_{j}(T)}=\sqrt{\frac{\epsilon_{\text {bulk }, i}}{\epsilon_{\text {bulk }, j}}}
$$

agree quite well with the ratios measured experimentally, as presented in Table (6.1). The linear dependence of $\operatorname{rms}(T)$ on $\sqrt{\epsilon_{b u l k}}$ is shown in Fig. (6.7).

\subsection{Conclusion and outlook}

In this Chapter it was shown that the temperature oscillations in the middle of the cell are sensitive to the shape of the container. As a plausible explanation, based on the experimental measurements, the different properties of the large scale circulation, particularly its life time, is offered. In order

Table 6.1: Comparison of the ratios of the rms' in given experimental setups calculated from (6.3) and the ones measured directly.

\begin{tabular}{ccc}
\hline & Eq. (6.3) & direct measurement \\
\hline leveled cube, inclined cube & 0.73 & 0.67 \\
leveled cube, inclined cylinder & 0.59 & 0.57 \\
leveled cube, leveled cylinder & 0.55 & 0.53 \\
inclined cube, inclined cylinder & 0.81 & 0.85 \\
inclined cube, leveled cylinder & 0.76 & 0.80 \\
inclined cylinder, leveled cylinder & 0.94 & 0.94 \\
\hline
\end{tabular}


to completely understand the phenomena and find theoretical expressions for the scaling of the root mean squares of temperature, experiments which concentrate on the detailed properties of the large scale flow in differently shaped containers should be performed. 


\section{Chapter 7}

\section{Summary}

By means of experimental methods the coherency on different scales was studied in turbulent Rayleigh-Bénard convection.

On the length scale of the boundary layers a new type of coherent structures was discovered. The structures were visualized using an electrochemical method which enables visualization in the very vicinity of the plates. Further, the statistical properties of the structures were analyzed. Finally, two possible mechanisms of their formation were proposed - the first is based on the assumption that they result from Rayleigh-Taylor instability and that their separation is periodic in the direction perpendicular to the large scale mean flow, and the second connects their formation to a stochastic process excluding periodicity in the separation. Both theories give good agreement with the experiment and it will be necessary to make measurements on a wider range of control parameters to make a final decision on the mechanism. As the particular visualization method used in this work is applicable only in water as the working fluid, alternative measurement methods were proposed.

On the large length scale - the scale of the container — the study of self organization in the turbulent flow was performed by means of simultaneous temperature measurements at different places in the container. Different regions of the flow could be detected as well as alternating temperature oscillations of the lower and upper part of the flow.

Using measurements of spatial correlations of the temperature field two length scales have been determined in the bulk. These two scales agree with the sizes of coherent structures visualized using thermochromic liquid crystals. It has been shown that the smaller of the two structures originate mostly from the detached thermal boundary layer, but some may also form in the bulk as passive scalar fronts. It is striking that in the hard turbulence régime the flow is dominated only by two length scales and not by a broad range of scales.

Measuring the spatial correlation (which is equivalent to the spatial 
power spectrum) and temporal power spectra at fixed points it was shown that the Taylor hypothesis is not valid. The consequence is that temporal spectra, obtained from the single probe fixed in space, do not provide the same information as the spatial correlation, so that it is necessary to measure both to reveal the properties of the flow.

Finally, using the observation of the visualized small scale structures and the measurements of the temperature fluctuations used in the study of the large scale coherency, the influence of the container shape on the flow was investigated. The experiments were performed in the containers with shapes mostly used in the study of Rayleigh-Bénard convection - a cylinder and a cube. The important differences in the velocity field of the large scale wind as well as in the temperature fluctuations in the fluid core were found. It is important to be aware of these differences as the measurements in cubical and cylindrical containers in the literature are mostly compared without regard to the shape. Additionally, a source of the difference and a hint for the scaling relations for the fluctuations were proposed. 


\section{Appendix A}

\section{Technical data}

In this Appendix technical data about the equipment and material used in the experiments is listed.

\section{Thermistors}

For all temperature measurements thermistors produced by the company "Thermometrics, Inc.", type AB6C8-BR14KA103K were used. The thermistors are of NTC ("negative temperature coeficient") type, that is, their resistance decreases as the the temperature increases. According to the method by which electrodes are attached to the ceramic body the thermistors belong to the bead type thermistors which have leadwires directly sintered into the ceramic body. The body is encapsulated in a glass coating. The other characteristics of the thermistors are:

- zero-power resistance at $25^{\circ} \mathrm{C}: 10 \mathrm{k} \Omega \pm 10 \%$

- nominal diameter of the body: $0.36 \mathrm{~mm}$

- nominal diameter of the leads: $0.03 \mathrm{~mm}$

- thermal time constant ${ }^{1}: 14 \mathrm{msec}$

\section{PCI Bus Data Acquisition Board}

The board was used to measure voltage drop on the Wheatstone bridge and transfer the data to computer. It is product of "Keithley Instruments, Inc.", type KPCI-1801HC with software configurable 64 single-ended or 32 differential input channels and software configurable individual gains for each

\footnotetext{
${ }^{1}$ The thermal time constant is the time required for a thermistor to change $63.2 \%$ of the total difference between its initial and final body temperature when subjected to a step function change in temperature under zero-power conditions.
} 
input channel. In all measurements differential inputs were used to reduce noise to signal ratio according to the recommendations of the producer. Normally, the range of measurements was $0-20 \mathrm{mV}$. The board represents a unipolar signal as 12 bit number, which ensures the resolution of $4.9 \mu \mathrm{V}$. For this range the maximum throughput is 75 ksamples/s which is three orders of magnitude more then actually needed in a typical experiment.

\section{Wheatston bridges}

Wheatston bridges were self produced using two resistors with nominal values $10 \mathrm{k} \Omega$ and $20 \mathrm{k} \Omega$ and a potentiometer produced by "Bourns", type 3590S2-203. The exact values of each resistor were determined. Before each measurement the exact value of the potentiometer resistance was calculated using a known resistance in the place of the thermistor. The bridges were put in a metal chassis for shielding against the noise. The bridges were supplied with a voltage of approximately $1 \mathrm{~V}$.

\section{$p H$ indicator}

For visualization of the streaks in the boundary layers $p H$ indicator Thymol Blue was used, provided by "Sigma-Aldrich Co." This is actually Thymolsulfonphthalein with the chemical formula $\mathrm{C}_{27} \mathrm{H}_{30} \mathrm{O}_{5} \mathrm{~S}$.

\section{Camera}

Interval photographs of the streaks were taken with 5 Megapixel camera Coolpix 5700 produced by "Nikon". Following are some more important characteristics of the camera:

- CCD sensor: high resolution 0.67" CCD; total number of pixels: 5.24 Megapixel

- maximal picture size: $2560 \times 1920$ pixel

- lenses: Nikkor-Zoom

- focus: 8.9 to $71.2 \mathrm{~mm}$ (corresponds to 35 to $280 \mathrm{~mm}$ Leica format)

- apperture: 2.8 to 4.2

- storage media: CompactFlash Cards Type I and II, Microdrive (512MB, $1 \mathrm{~GB})$

- data formats: JPEG, TIFF, NEF and QuickTime movies 


\section{Heating and cooling}

The container was cooled from above using a cooling thermostat RC-6-CS produced by "Lauda":

- working temperature range: $-35-150{ }^{\circ} \mathrm{C}$, temperature can be regulated digitally using a potentiometer with the resolution $0.1^{\circ} \mathrm{C}$

- cooling capacity at $20^{\circ} \mathrm{C}: 0.3 \mathrm{~kW}$

- pressure pump: max. pressure 0.32 bar, $\max$ flow $20 \mathrm{l} / \mathrm{min}$

The lower plate was heated with two electrical film heaters produced by "Telemeter Electronic", model HK-5179-R50.4-L12-B glued to the outside of the plate. Each film has a size $101.6 \mathrm{~mm} \times 203.2 \mathrm{~mm}$ and a resistance $50.4 \Omega$. The heaters were supplied with constant power.

\section{Thermochromic liquid crystals (TLC)}

Thermochromic liquid crystals were used for the flow visualization in Chapter 5 (see also Appendix C). The crystals are microencapsulated TLC slurries $50-100 \mu \mathrm{m}$ with the application range from $15^{\circ} \mathrm{C}$ (red) to $25^{\circ} \mathrm{C}$ (blue). The crystals are a product of "Hallcrest Inc.". 


\section{Appendix B}

\section{Computer programs}

In this Appendix programs made for different purposes in the framework of the thesis are listed and explained to some details.

\section{B.1 Hardware Control}

RBScan The program is written in $\mathrm{C}++$ and has a graphical user interface. Its purpose is manifold:

- setup PCI Bus Data Acquisition Board (see Appendix A), start measurement and save the measured data (Fig. B.1),

- convert measured voltage to temperature data and save to file (in different possible formats) (Fig. B.2),

- perform Fourier transformation (Fig. B.3) and

- interface to other console programs for data analysis or hardware control (described bellow), Fig. (B.4).

volt_front, res_front, gpbi Console programs for measurement of voltage and resistance from the front panel (volt_front and res_front, respectively) and resistance or voltage from channels of the rear modules (gpbi) of "Keithley" multimeter type 2700.

read_keithley Reads measured data from the Keithley 2700 Multimeter and saves it to computer disk in text format suitable for further analysis. The console program is written in $\mathrm{C}$.

\section{B.2 Data Analysis}

coherence Console $\mathrm{C}$ program for calculating coherence and phase shift of temperature oscillations in two points in the convection box (Section 5.3.1). 


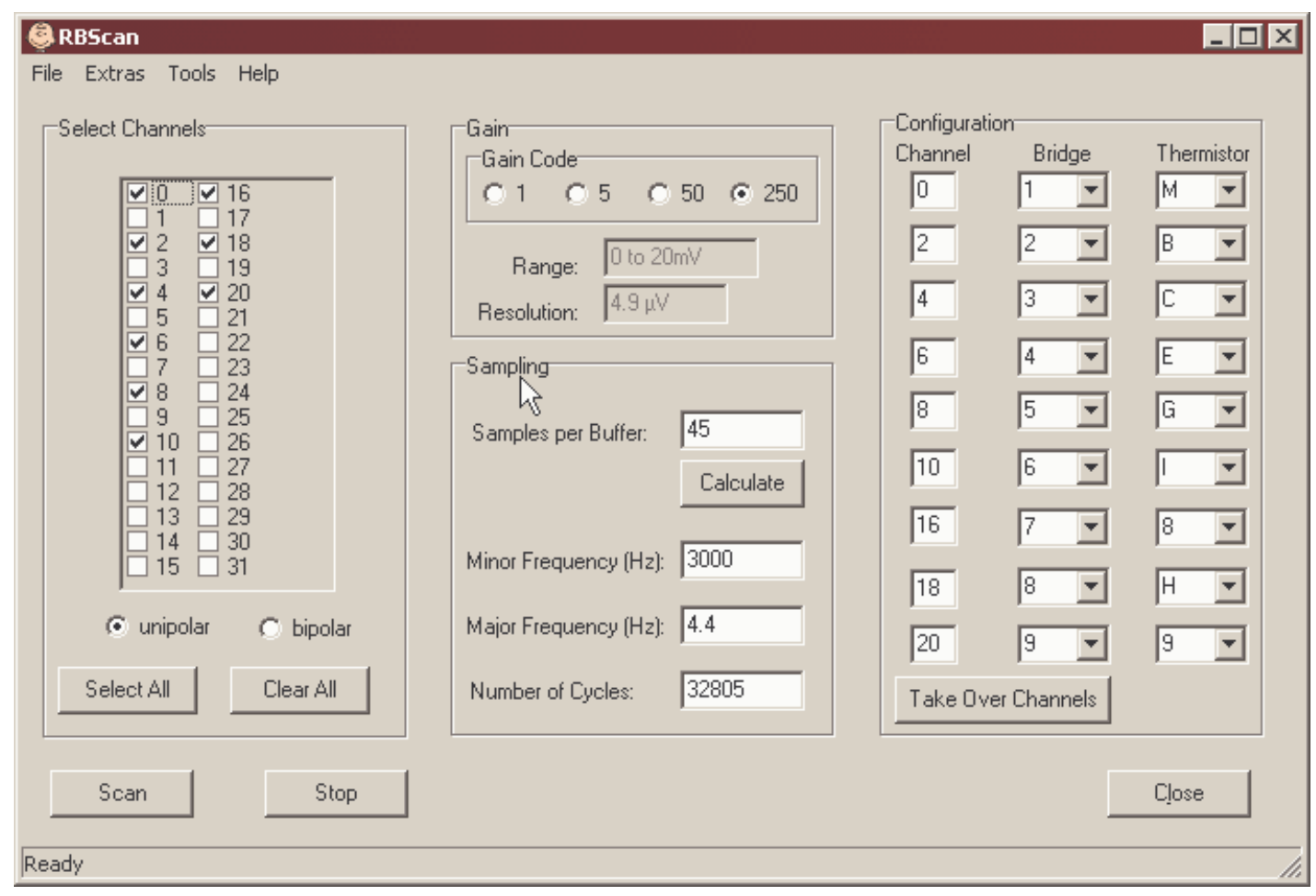

Figure B.1: RBScan, setup window.

crosscorelation, corr_space Console C programs for calculating temporal and spatial correlations.

eigenmodes Console $\mathrm{C}$ program for calculating modes and its spectra as defined in Section 5.3.2.

fourier Console C program for calculating power spectra of time series, with the possibility of low, high and band pass filtering.

StreakView The program takes two pictures and calculates the difference. It was used to get a clearer view of the streaks in the visualization experiments, Section 4.2. Typically, difference of a picture without streaks (background) and a picture with streaks was calculated, leaving in ideal case only the streaks. The actual results are pictures like the one in Fig. (4.2). The program has features of fine tuning contrast and threshold and calculating the position of a mouse click on the resulting picture. The graphical user interface is shown in Fig. (B.5).

length_conv The program converts distances determined from the digital images to real distances, based on calibration data. A cubic spline interpolation is done using a routine provided by Numerical Recipes (Press et al., 1995). 


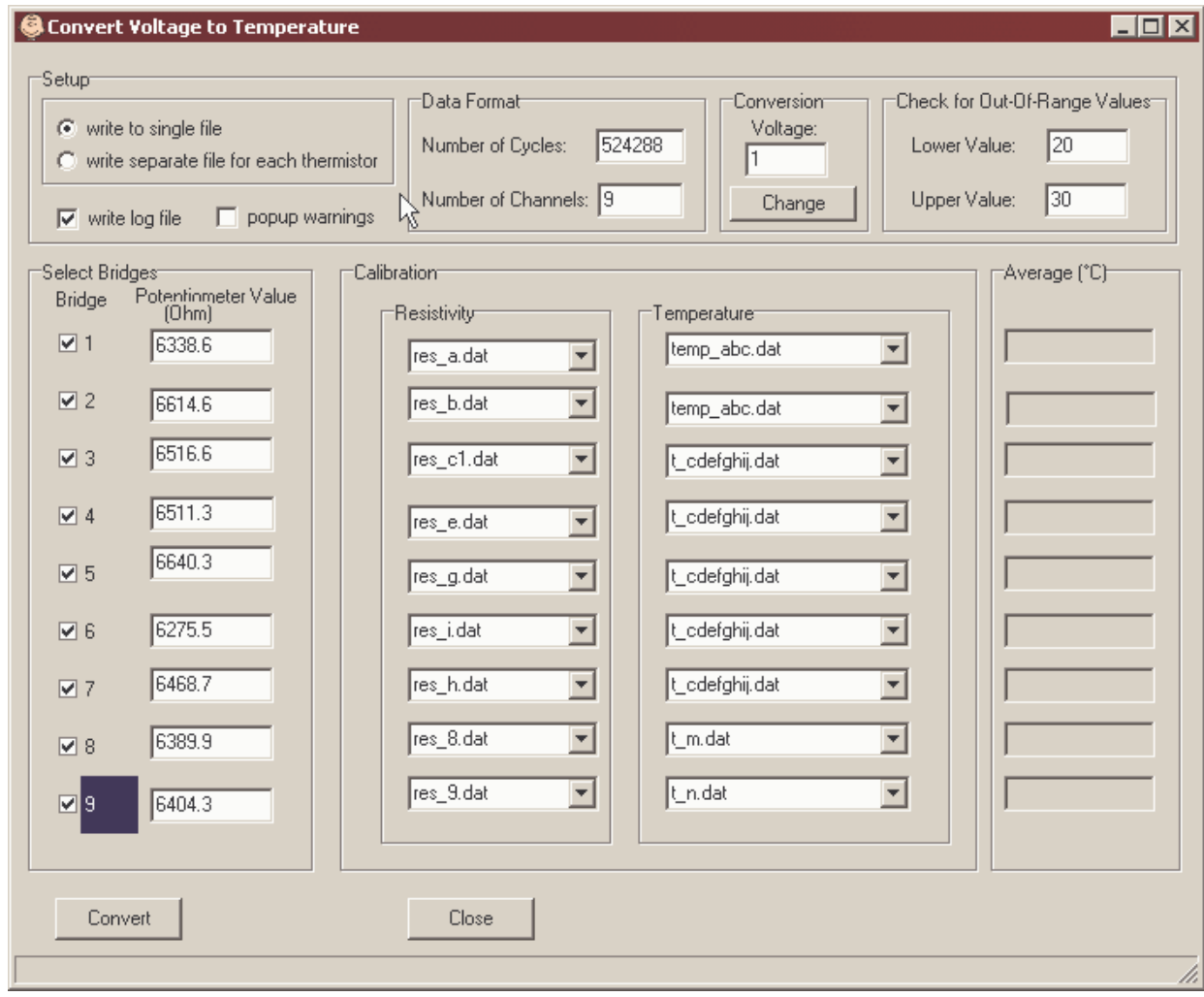

Figure B.2: RBScan, conversion of voltage measured on the Wheatstone bridges to temperature.

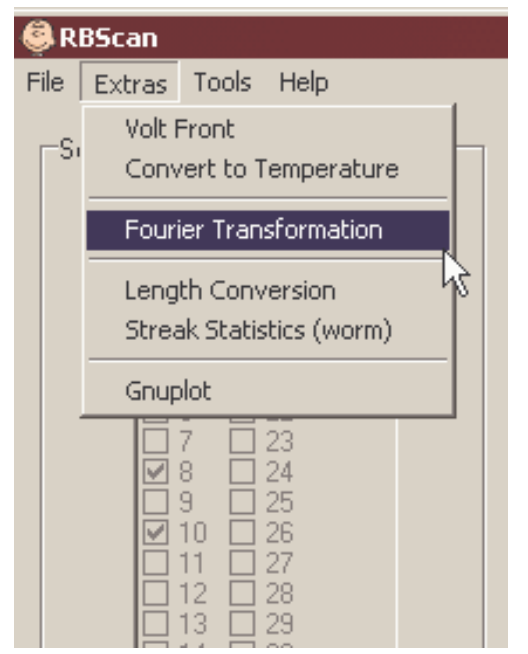

Figure B.3: RBScan as interface to other programs. 


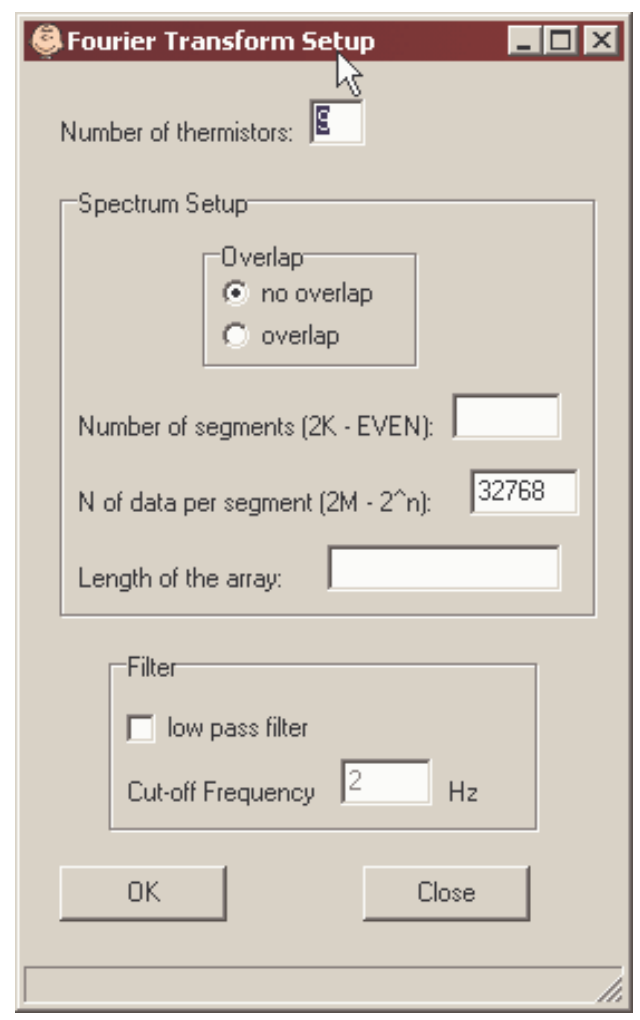

Figure B.4: RBScan, setup for Fourier transformation. 


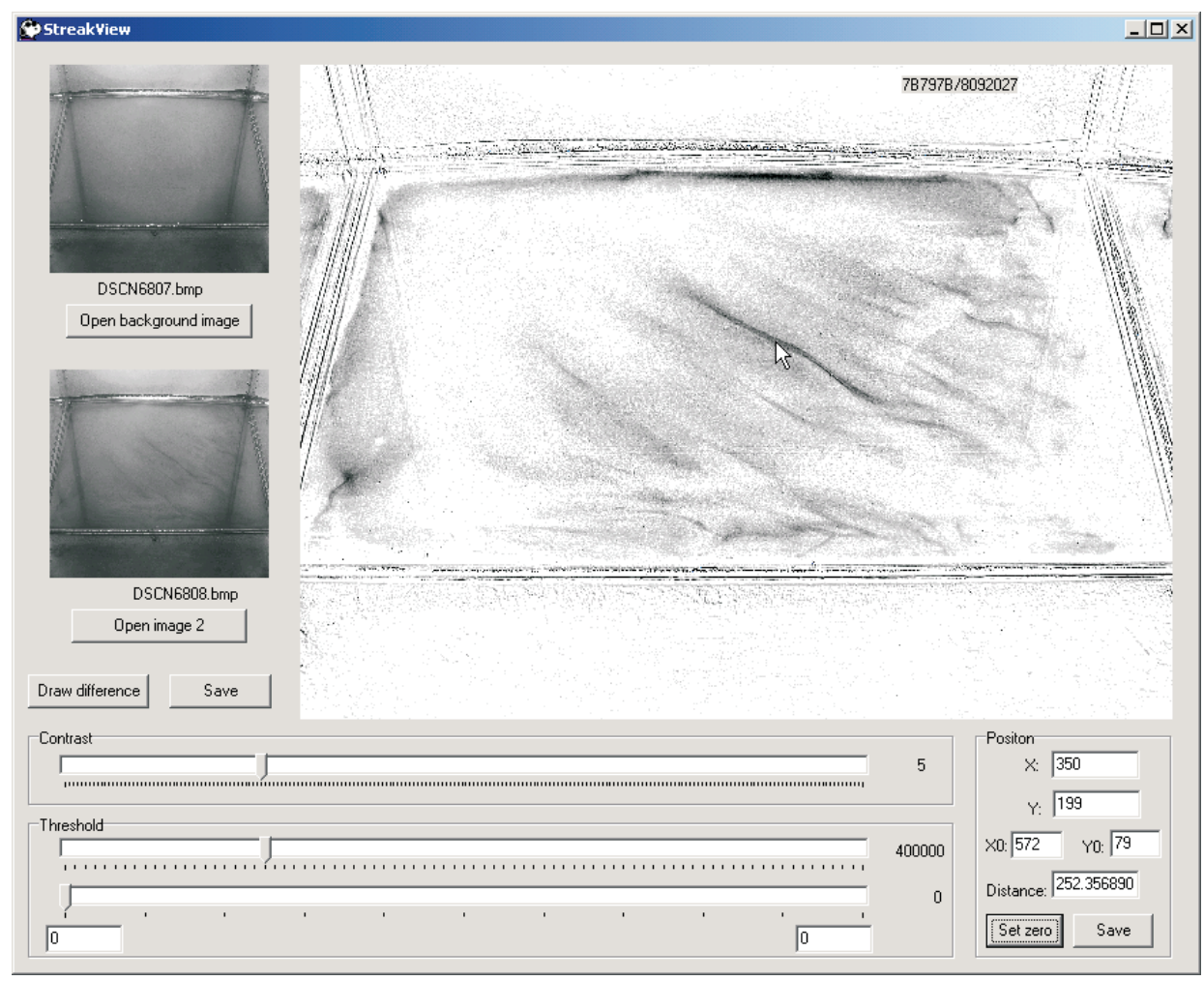

Figure B.5: StreakView graphycal user interface.

worm Console program written in $\mathrm{C}$ for calculating the histograms of streak separations and streak distribution in space.

poiss_streak Console program written in $\mathrm{C}$ for calculation of the number of streaks per photo (Eq. (4.10) and (4.11)).

rti Console program written in Fortran for linear stability calculations described in Section 4.3.1. 
Appendix C

Flow visualization with

thermochromic liquid crystals
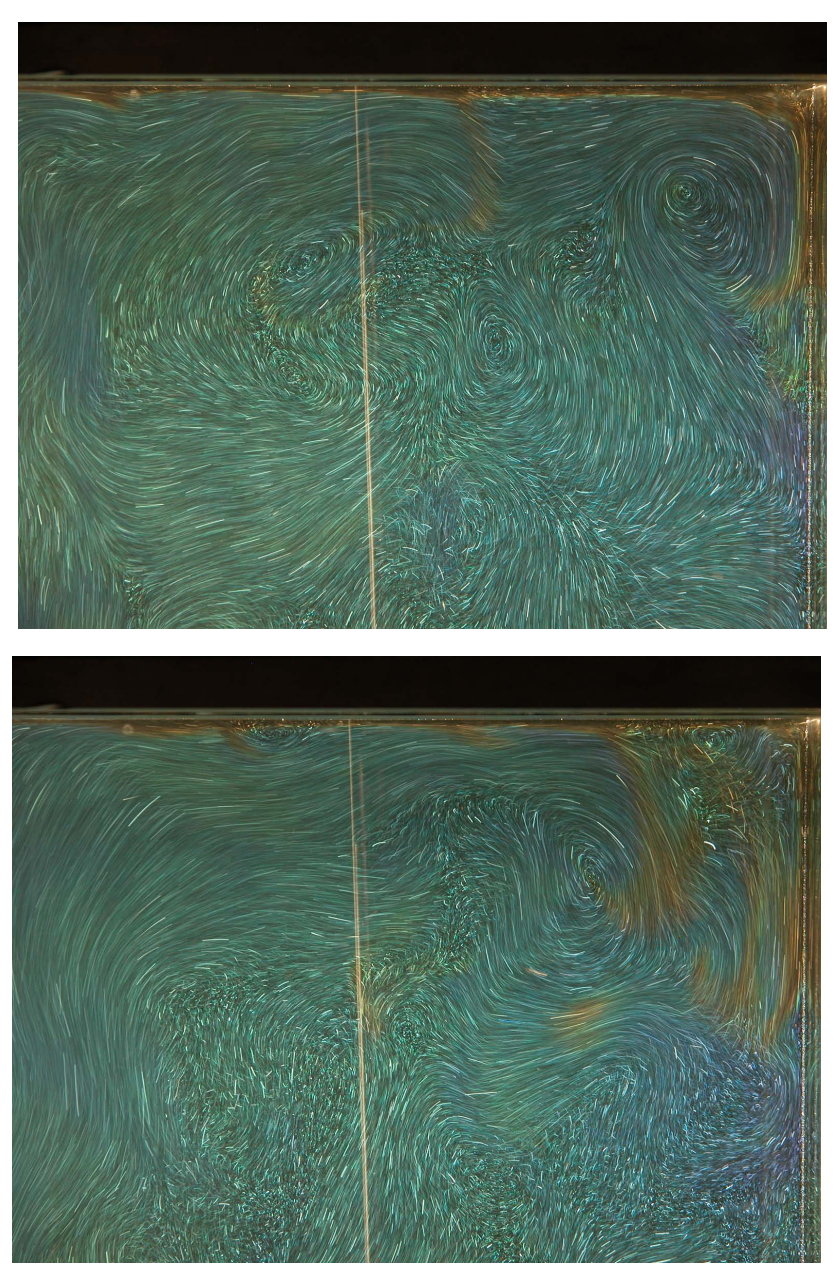

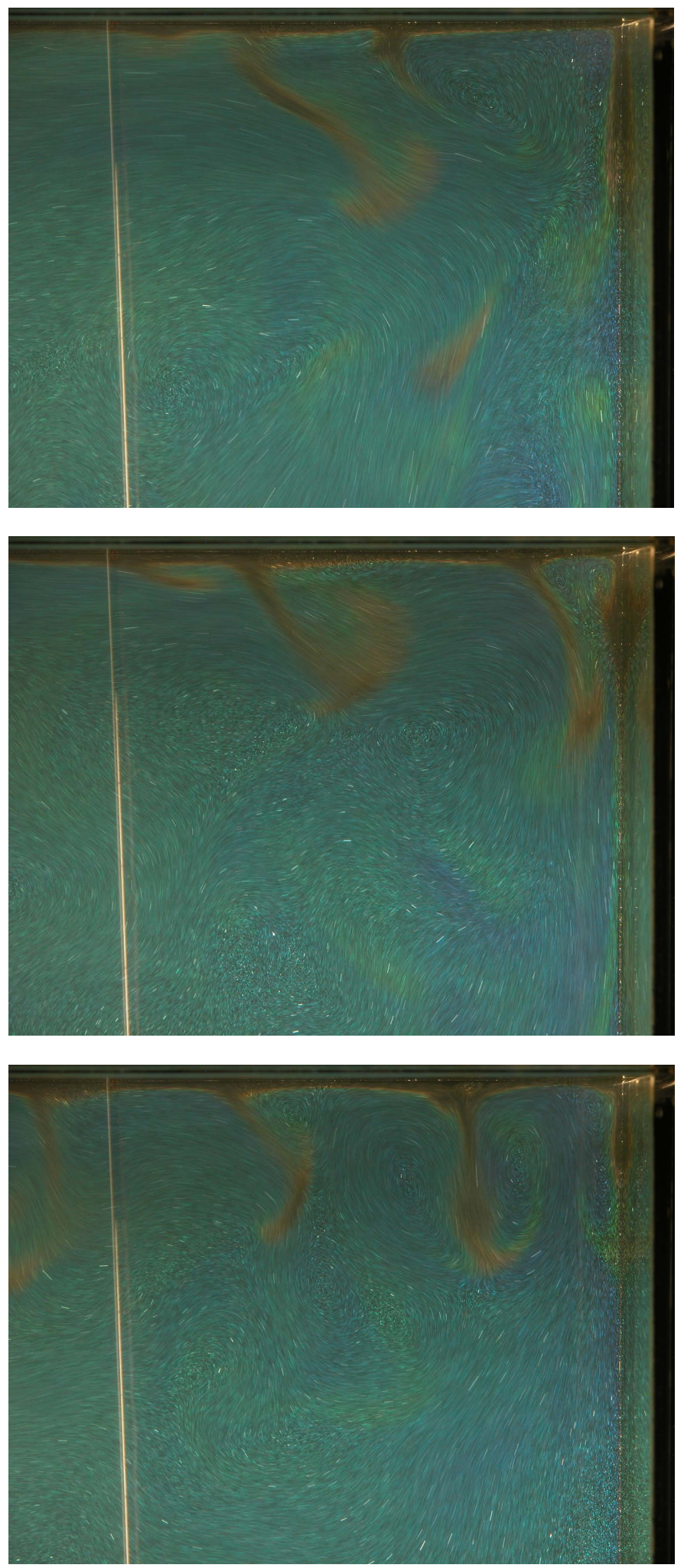

Figure C.1: Flow visualization in the part of the upper half of the cell. The bright vertical line is the thermistor holder. 


\section{Bibliography}

D. J. Baker, 1966, A technique for the precise measurement of small fluid velocities. J. Fluid Mech. 26, 573.

A. Belmonte and A. Libchaber, 1995, Thermal signature of plumes in turbulent convection: The skewness of the derivative. Phys. Rev. E 53, 4893.

H. Bénard, 1901, Les tourbillons cellulaires dans une nappe liquide transportant de la chaleur par convection en régime permanent. Ann.d. Chimie et de Physique 23, 62 .

J. Boussinesq, Theorie Analytique de la Chaleur, Vol. 2 (Gauthier-Villars, Paris, 1903).

F. H. Busse, 1978, Non-linear properties of thermal convection. Re. Prog. Phys. 41, 1930.

F. H. Busse, Transition to turbulence in Rayleigh-Bénard convection. In Hydrodynamic Instabilities and the Transition to Turbulence, edited by H. L. Swinney and J. P. Gollub, vol. 45 (Springer-Verlag, Berlin, Heidelberg, New York, 1981).

K. Butler and B. Farell, 1992, Three-dimensional optimal perturbations in viscous shear flow. Phys. Fluids A: Fluid Dyn. 8, 1637.

B. Castaing, G. Gunaratne, F. Heslot, L. Kadanoff, A. Libchaber, S. Thomae, S. Zaleski and G. Zanetti, 1989, Scaling of hard turbulence in Rayleigh-Bénard convection. J. Fluid Mech. 204, 1.

S. Chandrasekhar, Hydrodynamic and Hydromagnetc Stability (Dover Publications, Inc., New York, 1981), 654 pp.

E. S. C. Ching, K. W. Chui, X.-D. Shang, X.-L. Qiu, P. Tong and K.-Q. Xia, 2004, Velocity and temperature cross-scaling in turbulent thermal convection. Journal of Turbulence 5, 1 .

Z. Daya and R. Ecke, 2001, Does turbulent convection feel the shape of the container? Phys. Rev. Lett. 87, 184501. 
D. Funfschilling and G. Ahlers, 2004, Plume motion and large-scale circulation in a cylindrical Rayleigh-Bénard cell. Phys. Rev. Lett. 92, 194502.

J. P. Gollub and H. L. Swinney, 1975, Onset of turbulence in a rotating fluid. Phys. Rev. Lett. 35, 927.

D. Gray and A. Giorgini, 1976, The validity of the Boussinesq approximation for liquids and gases. Int. J. Mass Heat Transfer 19, 545.

S. Grossmann and D. Lohse, 2000, Scaling in thermal convection: a unifying theory. J. Fluid Mech. 407, 27.

S. Grossmann and D. Lohse, 2004, Fluctuations in turbulent RayleighBénard convection: The role of plumes. Phys. Fluids 16, 4462.

J. O. Hinze, Turbulence (McGraw-Hill, New York, 1959), (null) pp.

M. Holzer and E. D. Siggia, 1994, Turbulent mixing of a passive scalar. Phys. Fluids 6, 1820.

R. Krishnamurti, 1970a, On the transition to turbulent convection. Part 1. The transition from two- to three-dimensional flow. J. Fluid Mech. 42, 295.

R. Krishnamurti, 1970b, On the transition to turbulent convection. Part 2. The transition to time-dependent flow. J. Fluid Mech. 42, 309 .

R. Krishnamurti and L. Howard, 1981, Large-scale flow generation in turbulent convection. Proc. Nat. Acad. Sci. USA 78, 1981.

K.R.Sreenivasan, A. Bershadskii and J. Niemala, 2002, Mean wind and its reversal in thermal convection. Phys. Rev. E 65, 056306.

Lord Rayleigh, 1916, On convection currents in a horizontal layer of fluid, when the higher temperature is on the under side. Phil. Mag. (6) 32, 529.

V. L'vov, 1991, Spectra of velocity and temperature fluctuations with constant entropy flux of fully developed free-convective turbulence. Phys. Rev. Lett. 67, 687.

G. Marquart, H. Schmeling and A. Braun, 1999, Small-scale instabilities below the cooling oceanic lithosphere. Geophysical Journal International 138,655 .

J. Niemela, L. Skrbek, K. Sreenivasan and R. Donnelly, 2001, The wind in confined thermal convection. J. Fluid Mech. 449, 169.

A. Oberbeck, 1879, Über die Wärmeleitung der Flüssigkeiten bei der Berücksichtigung der Strömungen infolge von Temperaturdifferenzen. Ann. Phys. Chem. 7, 271. 
W. H. Press, S. A. Teukolsky, W. T. Vetterling and B. P. Flannery, Numerical Recipes in C: the art of scientific computing. 2. ed. (Cambridge University Press, Cambridge, New York, Melbourne, 1995), 994 pp.

X.-L. Qiu and P.Tong, 2002, Temperature oscillations in turbulent RayleighBénard convection. Phys. Rev. E 66, 026308.

X.-L. Qiu and P. Tong, 2001, Onset of coherent oscillations in turbulent Rayleigh-Bénard convection. Phys. Rev. Lett. 87, 094501.

X.-L. Qiu and K.-Q. Xia, 1998, Spatial structure of the viscous boundary layer in turbulent convection. Phys. Rev. E 58, 5816.

S. Reddy and D. Henningson, 1993, Energy growth in viscous channel flows. J. Fluid Mech. 252, 209.

M. Sano, X. Z. Wu and A. Libchaber, 1989, Turbulence in helium-gas free convection. Phys. Rev. A 40, 6421.

T. Segawa, A. Naert and M. Sano, 1997, Matched boundary layers in turbulent rayleigh-bénard convection of mercury. Phys. Rev. E 57, 557.

B. Shraiman and E. D. Siggia, 2000, Scalar turbulence. Nature 405, 639.

L. Skrbek, J. Niemela, K. Sreenivasan and R. Donnelly, 2002, Temperature structure functions in the bolgiano regime of thermal convection. Phys. Rev. E 66, 036303.

C. R. Smith and S. P. Metzler, 1983, The characteristics of low-speed streaks in the near-wall region of a turbulent boundary layer. J. Fluid Mech. 129, 27.

H. Tennekes and J. Lumley, A first course in turbulence. 8. ed. (The MIT Press, Cambridge, Massachusetts, London, 1982), 300 pp.

A. Tilgner, A. Belmonte and A. Libchaber, 1993, Temperature and velocity profiles of turbulent convection in water. Phys. Rev. E 47, 2253.

D. J. Tritton, Physical Fluid Dynamics. 2. ed. (Oxford University Press, New York, 1992), 519 pp.

Z. Warhaft, 2000, Passive scalars in turbulent flows. Annu. Rev. Fluid Mech. 32, 203.

H.-D. Xi, S. Lam and K.-Q. Xia, 2004, From laminar plumes to organized flows: the onset of large-scale circulation in turbulent thermal convection. J. Fluid Mech. 503, 47.

Y.-B. Xin, K.-Q. Xia and P. Tong, 1996, Measured velocity boundary layers in turbulent convection. Phys. Rev. Lett. 77, 1266. 
S.-Q. Zhou and K.-Q. Xia, 2001, Scaling properties of the temperature field in convective turbulence. Phys. Rev. Lett. 87, 064501.

G. Zocchi, E. Moses and A. Libchaber, 1990, Coherent structures in turbulent convection, an experimental study. Physica A 166, 387. 



\section{Acknowledgments}

Although a $\mathrm{PhD}$ thesis officially results from an "independent scientific work", there are lots of people who directly or indirectly supported me during the last four years and made this work possible. Here I would like to thank them all.

Firstly, I would like to thank my supervisor Prof. Andreas Tilgner. When knocking on his office door with a problem and the question "have some time?" I never ever got the answer "no". His advises and discussions were for me of great importance and I can hardly imagine a better mentor.

In every experimental work a great deal of success depends on the technical setup. Here I have to thank Mr. Wilfried Steinhoff who did a great job by building most of the mechanical parts for the experiment and Dr. Martin Leven for detailed explanations and help with different problems with electronics I had.

A "thank you" deserves Dr. Arno Ickler for proof reading the thesis and valuable comments and discussions about my work. His wide professional knowledge and friendly attitude made me enjoy his company. My dear friend and office mate Ellen Hage I want to thank for proof reading the thesis, for all the pleasant professional and private chats, for teaching me German and for being one of the persons who made my stay in Germany such a nice experience.

I thank the members of my Research Group for Geophysical Fluid Dynamics as well as all the other members of the Geophysical Institute for pleasant atmosphere and motivating discussions on different areas in physics.

Finally, without my wonderful wife Tatjana I would not be where I am. Thank you Tanja for your support through all these years! 



\section{Lebenslauf}

\section{Persönliche Daten}

Name:

Geburtsdatum:

Geburtsort:

Staatsangehörigkeit:

\section{Schulbildung}

$07 / 1979-06 / 1987$

$07 / 1987-06 / 1991$

\section{Studium}

10/1991-04/1999

$01 / 2002-12 / 2005$
Tomi Haramina

4. Mai 1972

Zagreb, Kroatien

kroatisch

Grundschule, Zagreb

Gymnasium, Zagreb; Abschluss: Abitur

\section{Berufliche Tätigkeit}

$03 / 2000-09 / 2000$

Softwareentwicklung bei "Emporion", Zagreb

$09 / 2000-12 / 2001$

Softwareentwicklung bei "Siemens", Zagreb, Graz

$01 / 2002-12 / 2005$

Studium der Physik an der Universität Zagreb, Fakultät für Naturwissenschaften und Mathematik; Abschluss: Dipl. Phys.

Promotion mit dem Thema: "Coherent structures in turbulent Rayleigh-Bénard convection."

Wissenschaftlicher Mitarbeiter an der Uni Göttingen, Institut für Geophysik 